\title{
AJUSTAMENTO DE ALGUNS MODELOS EXPONENCIAIS A DADOS DE CRESCIMENTO DA CANA-DE-ACUUCAR
}

\author{
PAULO DE OIIIVFIRA \\ Estatisitico
}

Or ientadior: pror. Tr. ROBERTO STMIONATO MORAFS

Dissertação apresentada à Escola Superior de Agricul tura "Luiz de Gueiroz", da Universidacie de Sxio Faulo: para obtençáco do t.f tulo de: Mestre em Agronomia, Áreá de concentraç̃o: Estatistica Fxperi mentação Agronomica.

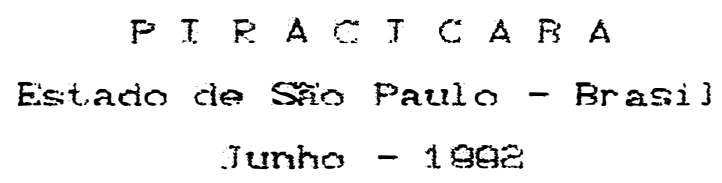


Oliveira, Paulo de

$048 a$

Ajustamento de alguns modelos exponenciais a dados de crescimento da cana-deaçúcar. Piracicaba, 1992.

89p.

Diss. (Mestre) - ESALQ

Bibliografia

1. Cana-de-açúcar - Crescimento - Modela matemático 2 . Modelo matemático I. Escola Superior de Agricultura Luiz de Queiroz, Piracicaba.

CDD 533.61 


\section{AJUSTAMENTO DE ALGUNS MODELOS EXPONENCIAIS A DADOS DE CRESCIMENTO DA CANA-DE-ACUCAR}

PALILO DF OII VFIRA

Aprovicies em: OE $/ 11 / \mathrm{SP}$

Comb gato jul gaciora:

Pror Dr. Roberto Simionato Moraes

FSALONSP

pror a bra shetila Zambel to de Pinno

TB $/$ UNFSP

Pror a do clarice Garcia Borges Remetrio

ESAI Q UUSP

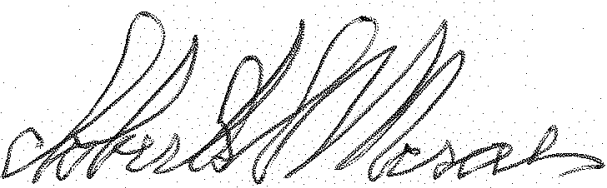

Prof. Dr, ROBFRTO STMTONATO MORARS or ientacior 


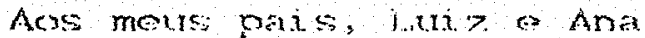

A minha exposen, Exri.

Aos meus itinos Rodrigo.

Pavi es, firumo o flago

Ixtelic:os 
iv.

AGRADECT MENTOS:

A Fimpressa de Peesquis Agroperuaria de Minas Gerais CEPAMTS e A Fscola Superior de Agricultura Hithz de

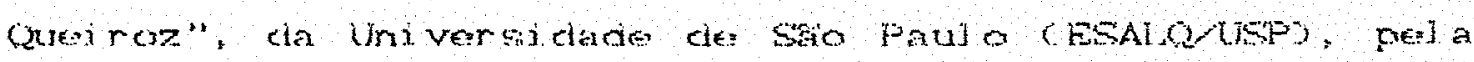
oporimalede concedida para a rantipacko do turso de

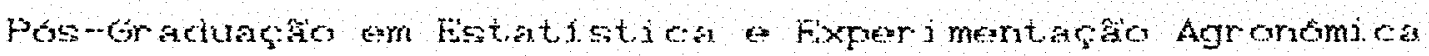

An laboratorio de Computarao Cientifica cloco da Universidacte Federat de Minas Gerals CUFMG que prermitiu o use de? seu computador, para processar as analises.

Acs professor Dr. Koberto simi onato Moraes, prela

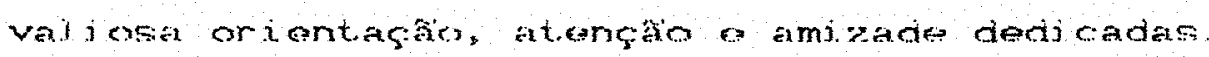

Aos professores o fumojonários do lkopartamento de Matematica e Fistatiotice da BSAl Q peios valiosos ensinamentos e convi vênei

Aos colegne dis aren de Computagio de PPAMT pelo ipria e incertivo.

Ans colegas do curso, pei a convino bamzade.

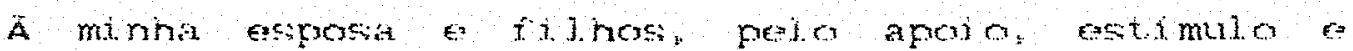
detis circtio. 


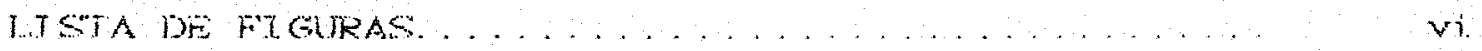

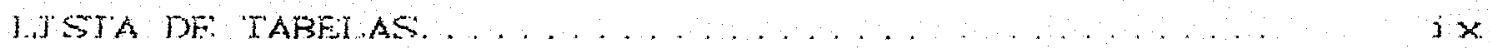

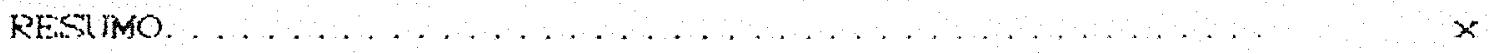

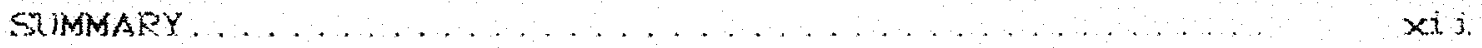

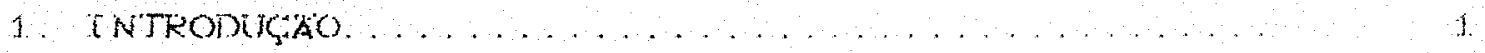

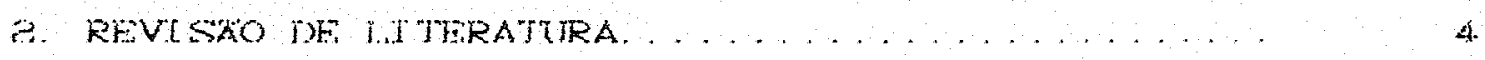

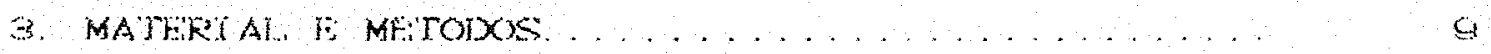

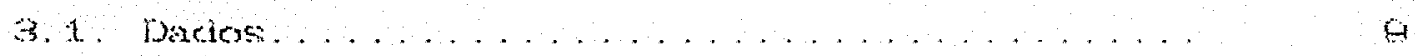

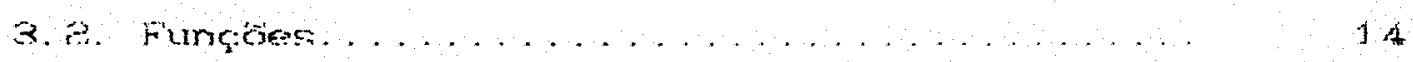

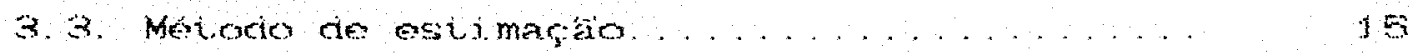

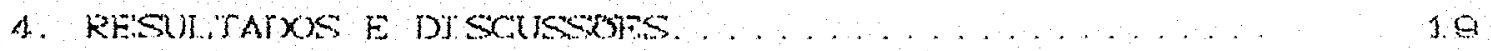

4. A Auste def furrase for dadro do

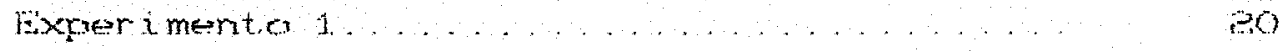

4. Ajuste das fucces are dados do

Fexper merteo

4. B. Ajusto das fumpes an dados dos

Experimentos $3,4, k, 6,7,8,9, \quad 36$

5. CONCWUSBES

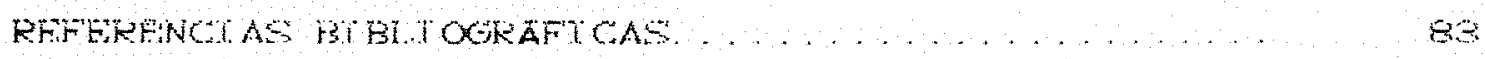

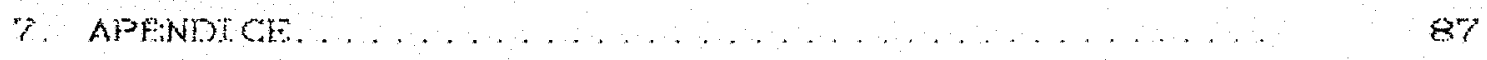


LISTA DF: FIGURAS

Fágina

Figura 1 - Diversas formas de curvas de

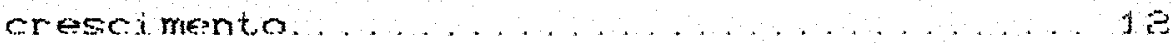

Fisfura a - Grafteos das curvas estimadas etraves da funçács logística (a) sobrepostos aos pontos obser vacios nos Fxperimentos $1,9,3,4 \ldots \ldots, 38$

Figura 3-Graficos das curvas estimadas atravers da functio logletica Ca sobrepostos aos pontos cheservado nos Experimentos $5,6,7,8, \ldots, 20$

Figura a - Graficos das ourvas estimadas através da runsto logist, ca cbo sohrepostor aos pontos onservado nos fxperimerton $1,9,3,4$

Figura g- Graficers das curvas ostimadac alravero da functoo logist oa (b) sobrepostos aos pontos orserevacies nos Exper mentos $5,6,7,8$

Figura o-- Graficos das ourvas estimadas atraves da funcalo logistica or sobrepostas aos pontos obser vadoe, nos fixper i mentors $1,9,3,4$

Figura 7 - oraficos das curvas estjmadas atraves da funf observados ros Fxper mentos $6,6,7,8, \ldots \ldots 45$

Figura 8 - Graficos das curvas estimadas atraves da runço kompertz COS sobrepostos aos pontos observados nos Exper mentos $1,6,3,4 \ldots \ldots \ldots 48$

Fjoura o- Graficos das ourvas estimadas alraves da funcia Gompertz cas sobrepostos aos pontos observados nos Fxperimentos $5,6,7,8 \ldots \ldots .49$

Figjura 10 - Gratcos das rurvas estumadas atraves da fungar (Kompert $>$ (e) sobrepostos ans porton cubser vados nos Exper i mentos $1,8,3,4$ 
Figura 11 ... Graficos das corvag ostimadas atraves da

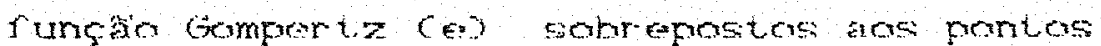
observedos nos Fxperimeritos $5,6,7,8 \ldots . . .6$

Figura ta-Griftche cas curvat estornadas atraves da funço mornmolecular sobrepostos ans portas cibservertest nos Fxperimenters $t, \theta_{1}, 4 \ldots, \ldots 4$

Fifgura 13 - Graflcos das curvas entimadas atravas da functio monomolecular sobreposton aos pontos onservadie nos Fxper imentos $9,6,7,8$.

Fjgura 1 - Grastcos das curvas ostumadas atraves da runfics Broly sonrepostos ans pontas orservados nos Fxpermentos o, $3,4, \quad 57$

Fjgura 15 Graficos das curvas estimadas atraves da funcho Hrony sobropostors ans portos ohservacios nos Fxperimentos $5,6,7,8, \ldots 8$

Flgur té - Graricos das ourvag netimanas atravos da functo logjstica modif bada sotrepostos ads pontos coservanos nos Experimentos $1,6,3,4,60$

Figur 17 - Graficos das curvas esthmads atraves da

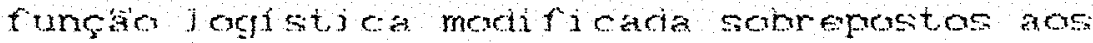
portos onservas nos Fxperimertos $6,6,7,8,61$

Fjgura le - Grafod das curvas ostumadas atraves da

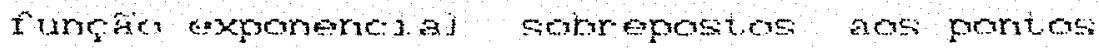

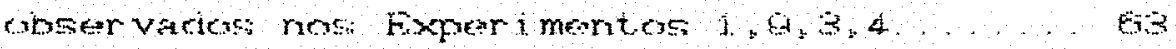

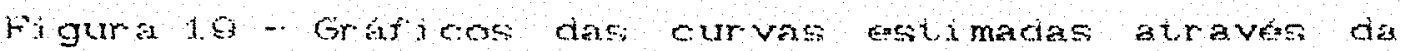
funça exponenotal formpoostos aos portos roservados nos Fxperjmentos b, $6,7,8, \ldots \ldots$

Figura to - Graficos das ourvas estimadas atraves da functio log-hnversa-general l zada sobraposuh aos pontos obsarvados nos Exper mentas

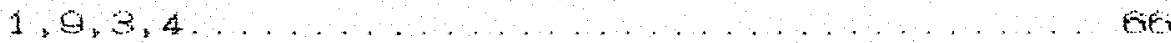


Ficjura a - Grafjoos das curvas estimadas atraves da fungan jog-inverse-generalizada sobrepostos aos pontos orservados nos fixperimentos

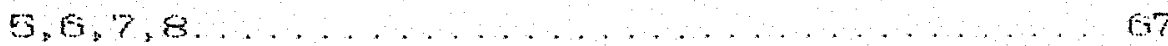

Figura 20 - Graricos das curvas ostimadas atraves da functo Richarde sobrepostos aos pontos observaciof nos Experimertas $1,9,3,4,60$

Figura 33 - Oraficos das rurvas tostimadas atravas da funcio Richards somepostos aos portos observarion nos Exporimerton $6,6,7,8,70$

Figura ta - Graficos dat rurvas estimadas atraves da fungto kelo sobrepostro aon pontos observadere nos Experimentos $1,9,8,4,77$

Figura H - Graficos daf curvas detimadas atraves da funga kel sobrepostos ans pontos

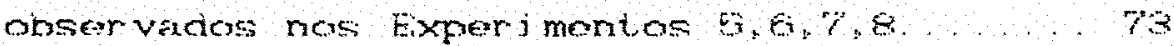


Tabela $\rightarrow$ Peso da cana-de-agnos em Kg/ha de masta verde ros Fopror merntos $2,4,6,6,7,8, Q$

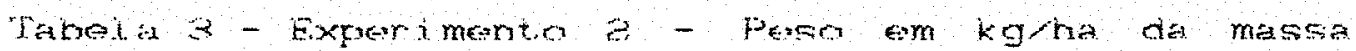
verce da cana-cie-arucar toana-soras.

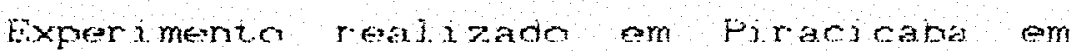

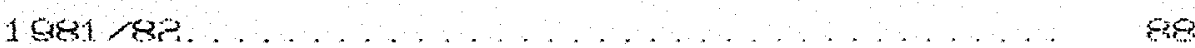




\title{
AJUSTAMENTO DE ALGUNS MODELOS EXPONENCIAIS A DADOS DE CRESCIMENTO DA CANA-DE-ACUCAR
}

\author{
Autor: PAUIOO DE OIIVETRA

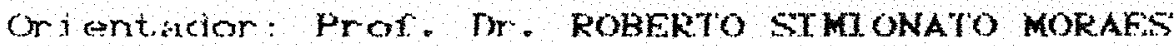

Resimo

A possibilidade de se estumar a curva de

crescimente da massa verde da cana-cle-arucar, como de

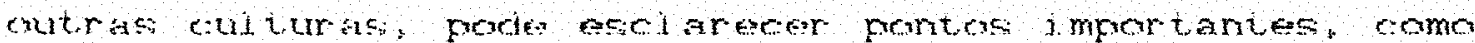

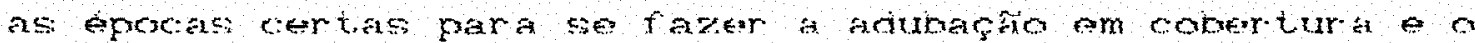
corte. O objetivo deste trabesho foj joentifiear modelos matematiciss representativos do ressolmento da

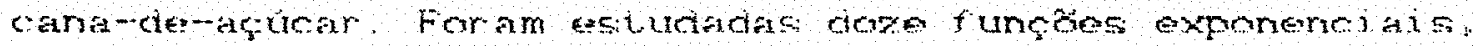
ajustadas a nove exper imentos, rada um produzindo uma

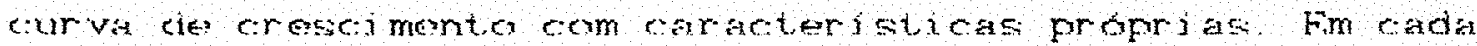
fungion, form onservacios as sogur ntes portoss. grau de

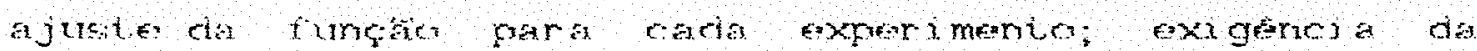

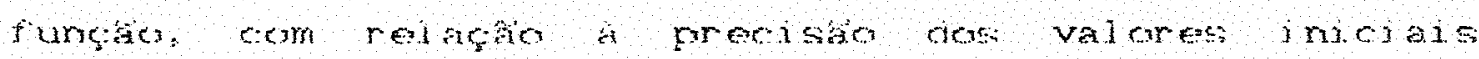
atribuldes acs pear ametros, para se atingir a convergencia; numero de iteraçes ate atingir a convergencja. A

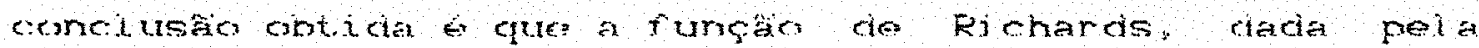


expressac $y=\beta_{\alpha}\left(1+\beta_{1} e^{-\beta} x, \gamma n\right.$, fol a que melhor se

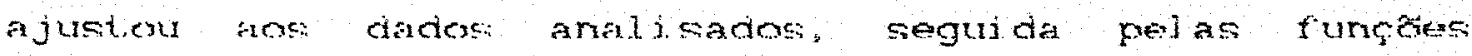

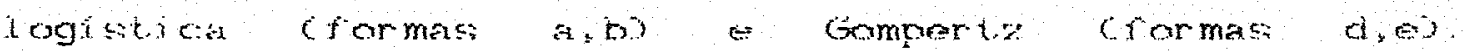
Diferentemente de Richards, neste tratelino n fol

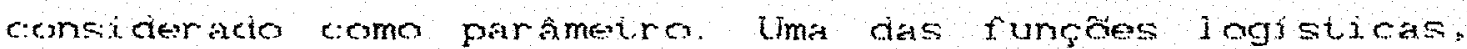

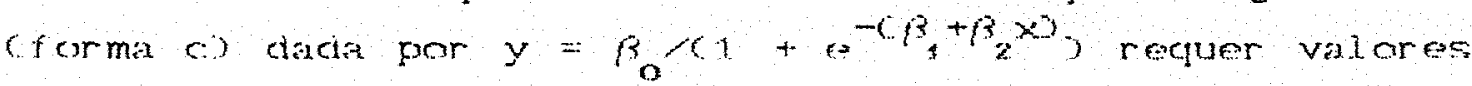
inicial muto proximos dac ost mativas dos parametros,

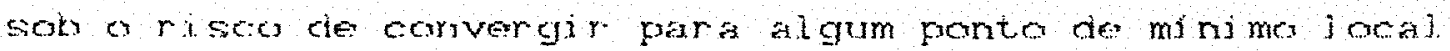


×i.j.

\title{
FITTING OF SOME EXPONENTIAL MODELS TO DATA OF SUGAR-CANE GROWING
}

Author: PAUIO DF OKTVETRA

Adviser WROF. DK. ROFFRTO STMTONATO MOKAFS

Simmerry

\begin{abstract}
The curve representing the growth and massa procicition ef sugar cane and other plarts offer a gorot potential for the appratsel of the bert tome for side

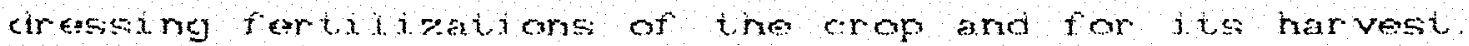
The cinjective of this work wat to identofy mathematsc model te regresenting thr growig of sugar-nare Twelve exponential funetichs were tuvdied fitted to nom expertments, acin we produetng one growng curve with $\mathrm{ts}$

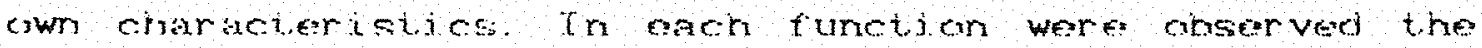
follewhol prontes degres of fjletho of the funetion lo

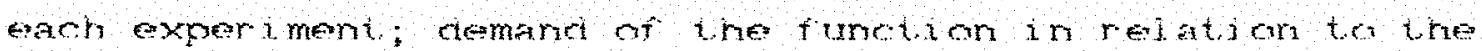

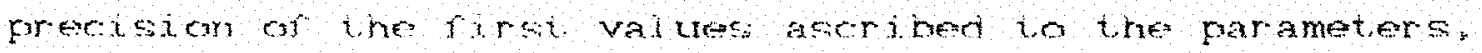
tor rean the convergency: the number of lnteractions to reach the canvergency The conclustor nhtalned to thet the

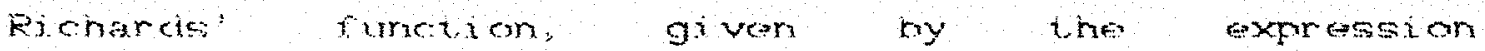


$\times i i j$

$y=\beta_{0}\left(1+\beta_{1} e^{-\beta} z^{-1 / n}\right.$, was the best fitted to the

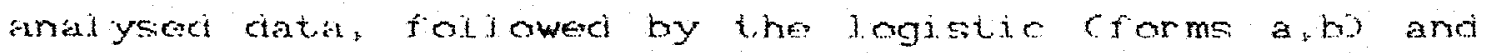
Gompertiz cforms d, to runetions. Thtferenti y from Eicherds. iri this work $n$ was considered a parameter. Cres of the legistic function crom a given by $y=\beta_{0},\left(1+e^{\cdots(\beta}+\beta, x\right)$, neescis starting values very near to the estimatess or the perameterg because ir not it car tend to somes pointes of local minimum. 


\section{INTRODUÇÃO}

Nos ultimos tempos, alem da produção de açucar e de alcool, a cana-de-açucar vem apresentando novas utilizaços para os produtos como o bagaç, o fermento, etc, ate entzo, considerados como residuos industriais inaproveitaveis, tornardo-se algurs deles, produtos de exportaçăo.

Com essa rova forte de riqueza, acrescida do aumento da representatividade como fonte renovavel de energia, que, segundo dados do ANUARIO ESTATISTICO DO BRASIL (AEB) - 1989 , passou de $7,51 \%$ em 1970 para $18,89 \%$ em 1987, sendo o bagaço responsavel por $35,99 \%$ do total da energia primaria consumida em 1990 CAEE, 1991 , a cana-de-açucar volta a merecer maior interesse por parte dos produtores.

Esse interesse reflete na busca de um conhecimento mals abrangente da planta e de seu cultivo. sempre tendo em vista a malor produçáo com menor custo possivel. Sem duvida, o caminho para se atingir este objetivo passa pelo completo conhecimento da pianta e seu cuitivo.

Os metodos quantitativos constituem uma ferramenta util, para ajudar no conhedimento, por exemplo. da forma de crescimento da pianta. THORMLEY C1976J derende a ldéla de que se podem usar os metodos quaritiativos no estudo das plantas, em especial sua fisiologia. 0 
conhecimento da curva de crescimento da planta esclarece. dentre outros, dois pontos de suma importancla que são 0 ponto de inflexão e a assintota superior.

Fonto de inflexáo - E o ponto em que se inicia o processo de desaceleraçäo no ritmo de crescimento da planta.

Assintota superior - E o ponto em que a planta atinge sua producao maxima e praticamente cessa o processo veyetativo. O comhecimento exato deste ponto e 1 mportante porquo determina a epoca economi camente viavel, para se etetuar o conte, aproveitando a capacidade de producáo maxima da planta no menor tempo possivel.

Fara descrever curvas de crescimerto, seja vegetal seja animal, un metodo quantitativo eficiente e o do uso de modelos de regressäo. As curvas de eresclmento tem a forma sigmoide, com uma assintota inferior colncldente com o el xo das abscissas Cprodução zeros e uma superior cproducăo maxi mas. As funçós mals indicadas para descrever curvas desse tipo säo as exponenciais.

o objetivo deste trabalho encontrar uma funça que mel hor descreva curvas de crescimento. Com 1550 , busca-se conseguir informaçes a respelto das estimativas dos parametros desta funça.

Assime possivel, antes mesmo de 1 niciar o plantio, fazer inferenclas e ter informaçbes 1 mportantes sobre a cultura daquele ano, como, por exemplo, a epoca de se fazer a adubaço en cobertura para obter a melhor resposta da planta e o momento economicamente otimo de se iniciar o corte.

A curva de crescimento da cana-de-açcar depende de varlas variavels, multas delas controlaveis. ate certo ponto, pelo agricultor, como por exemplo, a cultivar utilizada no plantio a aplicaçáo de 
3.

fertilizantes, 0 uso de defensivos, irrigaçăo. o conjunto de todas essas variaveis pode determinar varios tipos de curvas dessa cultura, dal a nescessidade de encontrar uma furção que se ajuste bem näo apenas a uma curva mas tambem à grande maloria de todos os tlpos possiveis, dentro das limitaçóses da cultura. 


\section{REVISAO DE LITERATURA}

0 estudo de funçóes, que descrevem curvas de crescimento, näo e recente. No seculo passado, VERHULST (1345), estudando o creselmerto de populaçoes humanas, ajustou a funçao loglstica y $-\beta_{0} / C_{1}+e^{-C \beta_{1}}+\beta_{2} \rho_{3}$.

As curvas de crescimento possuem caracteristicas proprias. Em geral, as funçoes que mel hor representam estas curvas săo as nå lineares, cujas estimalivas dos parametros sä alcançadas por processos lterativos mui to trabal hosos. For esta razao, houve sempre una grande preocupaça em desenvolver metodos eflelentes de estimacia dos parametros.

SIEVENS C1951) apresentou um metodo de estimação dos parametros da equaçăo y $=\alpha+\beta o^{x}$ e de outras formulas de regressá que der l vam da equaçao acl ma. por simples transformaçes, como, por exemplo, as formulas de regressáo de Gompertz e $109 i s t 10 a .0$ metodo simpliflea os calculos matematicos, principalmente se os valores de $x$ são igualmente espacados, ou espaçados conforme al guma regularidade padrso. Outros autores deserval veram metodos de estimaço dos parametros de funcóes näo 1 ineares. alguns deles se tornaram bastante conhecidos, como os metodos "Steepest-Descent", Newton, Gauss-Newton, Fletcher E POWE11.

MAROUARDT C 1963 ) desenvol veu um aigor 1 tmo para estimar parametros de funços räo ilneares, pelos 
minimos quadrados. 0 autor conclilou 0 metodo "Steepest-Descent" com o de Gauss-Newton, aproveitando 0 que de melhor apresenta cada um deles. Dessa forma, quando se esta longe da sol ução, o algor t tmo faz uso do metodo de "Steepest-Descent", para indicar a direção que o vetor dos parametros deve tomar, para atirigir a convergencia. E sabido que este metodo eflciente nestas circunstancias. Ao se aproximar da soluçáo, a direçáo do vetor e indicada pelo metodo de Gauss-Newton, que $\& 0$ melhor nesta situacaio.

NELDER (1961) \& OLIVER (1966) estudando a estimação dos parametros da função log1stica, concluiram que sao nescessar as poucas observacoes para se estimarem os parametros desta funçzo.

RICHARDS $(1999$ ) considerou a função de crescimento de von Bertalanf $y$ e atribul u aplicaçes para a extensão desta funça 0 autor af Ima que as tres funçöes de crescimento mais conhecidas săo:

$$
\begin{aligned}
\text { Monomolecular } & \left.=A C 1-b e^{-k t}\right) \\
\text { Autocatalitica } & =A C 1+b e^{-k t}, \\
\text { Gompertz } & W=A e^{-b e^{-k t}}
\end{aligned}
$$

CAUSTON (1989) desenvolveu um programa para - ajuste da função de Richards, dada pela expressao y $=\beta_{0} c_{1} \pm \beta_{1} e^{-\beta_{2} x_{y}-1 \mathrm{n}}$ e apresentou um procedimento para obtenção da estimativa de $\mathrm{r}$, a partir dos dados observados.

$$
\text { HOFFMANN \& THAME C1970) ajustaram as }
$$
funf̧es $10 g i s t 1 c a$ e de Gompertz a dados de crescimento de Pinus cariboea, variedade coribaec, e veriflcaram que a função de Gompertz se ajustou melhor aos dados, do que a funça $10 g 1 s t l c a$. Observaram, alnda, que a função logistica apresentou estimativas para a assintota 
superior, muito inferior aos dados observados. MISCHAN (1972) constatou o mesmo problema, quando estudou o ajuste da função logistica aos dados de crescimento ponderal de gado bovino. VIEIRA (1975) estudou as seguintes funçoes assintoticas sigmoides.

$$
\begin{aligned}
\text { Logistica } y & =A C\left(1+e^{-C B+C x)}\right. \\
\text { Gompertz } & v=e^{A-B C^{x}} \\
\text { Log-inv. generalizada } y & =A+B R^{1<x}
\end{aligned}
$$

Essa autora observou tambem que os ajustes das funçoes logistica e de Gompertz mostraram assintota superior abal xo de valores observados. Segundo a autora, o metodo de STEVENS (19S1), usado para estimar os parametros das funcoes, deve ser responsabilizado, em parte, pelo resultado. Verifleou tambem que, apesar desse problema, a funçáo de Gompertz descreveu melhor a curva de crescimento.

Estudando as funçses de Mtscherlich, logistica e de Gompertz, no ajuste a dados de crescimento de gado bovino, MI SCHAN $(1972$ ) conciuiu que a funçá de Sompertz y $-A B^{X}$ ajustou-se melhor e apresentou as estinativas do parametro $C$ muito constante para os 76 animals estudados.

ARRUDA et aIl (1873) propuseram o uso da funça $y=A+B R^{1 \alpha}, c o m b>O$ e $O<R<1$ nos estudos que visam descrever curvas de creseimento Segundo esses autores, esta e uma funçáo sigmolde, com assintota supertor de ordenada A + E, serido que a abscissa do porto de inflexio da curva e dada por -1 elnR 0 ajuste desta funça tol comparado ao de Gompertz y $=\mathrm{AB}^{\mathrm{C}}$. apresentando-se mel hor que $a$ da segunda, em um dos tres exper imentos estudacios. 
CORTARELLI $(1973)$ ajustou, dentre outras, a

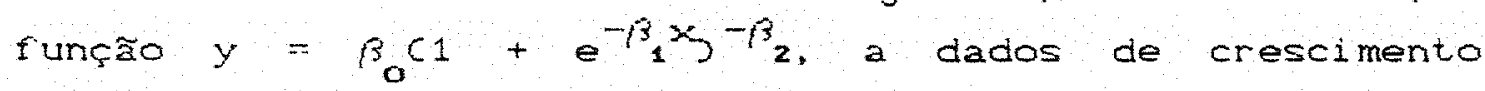
ponderal de bovino da raça Nelore.

Os metodos quantitativos constituem Instrumental eficiente para se estudarem varios fenomenos. inclusive o biologico THORNLEY (1970) afirma que a planta, em especial na sua fisiologia, esta apta cada vez mais, para a aplicaça de metodos quantitativos.

SPARROW $(1979$ ajuStou cinco modelos a dados de materla seca de forrageira, adubada com sels nivels de nitrogenio Un desses modelos estudados 101 de Reid. Alem dos criterlos de comparacao dos modelos, $\mathrm{R}^{2}$ e quadrado medio residual fol usado o de total de postos. 0 autor afirma, alrda, que um bom modelo nå deve ajustar-se bem apenas em alguns experimentos, nas razoavel mente bem ra maloria deles.

SILVA ( 1980 ) estudou metodos de estimação de parametros de modelos não lineares. Af irma que o sucesso ou rracasso na determinaça do ponto de minimo da Iuncáo do residuo depende da selecáo dos valores inicials dos parametros. Como nao existem metodos eficlentes para Se determinarem os valores inicials ldeals, a autorafixou intervalos para cada parametro, pesquisando as combinaços dos valores de cada um deles, optando pela combinaçáo que apresentou menor soma de quadrados dos desvios.

OLIVEIRA C 19863 comparou tres modelos ajustando-os a produção de soja, que recebeu seis niveis diferentes de calcario Como oriterio de comparaçăo dos modelos, a autora usou a analise de variancia, con o teste F; o coerldiente de determinaça ajustado para as graus de liberdade, o quadrado medio do desvio da regressáo, o criterio de informaçăo de AKAIKE e o total de postos. 
DRAPER \& SMITH (1981) cltam três métodos de estimação dos parametros de modelos não lineares: Linearização, "Steepest-Descent" e Marquardt. Este ultimo - considerado como sendo uma combinaça dos dols primeiros. Esses autores dão tambem uma interpretacá geometrica para a função dos residuos, ressaltando a importancia da escolha dos valores lnicials dos parametros e sugerem a utilização de todas as informaçoes ja existentes, para a escolha desses valores. Como exemplo de funçós não lineares, usadas para descreverem curvas de crescimento, citam a monomolecular, logistica e de Gompertz, dentre outras.

MAGNANI (1985) ajustou 0 modelo da segunda aproximaçăo de Mischerlich, a vinte e sete grupos de experimentos com a maturação da cana-de-açucar, expressos em pol co da cana. Estimou os parametros da funçá, pelos metodos de Newton, Gauss-Newton e Marquardt. Usou 0 metodo dos minimos quadrados para estimar os parametros da funcáo quadratica. Condulu que 0 metodo de Marquardt fol 0 mais eflelente 


\section{MATEKHAL, E MFTODOS}

3.1. Dados

A maior preocupaça deste trabal ho fol nấo apenas ajustar as funches aos dados de erescimento da cana-de-agucar, mas tambem verificar esse ajuste a diversas formas de crescimento, representadas por dados gerados a partir de um experimento real, denominado Experimento o. Fiste experimento o o resul tado obser vado em cana-soca de um trabal ho realizado pelo professor br. Foherto Simionato Moraes, em col aboraçío com tecni cos do Centro de Tecnologia da COPERSUCAR, em firacicaba, no pertode de junho de 1981 a feverejro de 1982 . Durante 0 periono do experimento for am tomadas nove medidas de peso, a intorvalos de aproximadamente un mes, sendo que em cada data tomaram-se quarenta e cinco observacies, de parcelas con trunta metros itreares, expressas em $\mathrm{kg}$ ha de massa verde?

Noste estudo, observados os dados, pode-se rotar que, ate a ut tima data, alnda não se tirha atingido 0 ponto de maximo. NF̈o se configurava uma situas̆ăo de estabilidade no crescimento da planta. por essa razáo foram acrescentados, empiricamente, dados de mais sete datas, com quarenta o cinco observaços cada no Experimento a buscando dotar a curva de uma jinha assintotica na parte superior, o que geral mente acontece com as curvas de cresci mentio. Os dados aparecem na jabela 3 no apondice. 
Como o processo de estimasáo dos parsmetros de runçose nå lineares $\leftrightarrow$, em geral, trabalhoso e demorado, optot-se por trabalhar, inicialmente, com um conjunto de apenes 10 pontos pareados $(x, y)$, onde $x$ representa a dade da rebrota da planta do Experimento $\mathrm{a} e$ y. o peso medilo de cada l dade. A esse conjunto de dados medidos do Fxperi mento $\approx$ denomi nou-se Experimento 1 , cujos dados aparecem na Tabela 1.

TARELA 1. Foperimento 1 - Feso medio da cana-de-asucar em $\mathrm{kg}$ ha de massa verde, do Experimento 2 no apendice.

\begin{tabular}{|c|c|}
\hline $\begin{array}{l}\text { Tdade da planta } \\
\text { Cmesess }\end{array}$ & $\begin{array}{l}\text { Feso da plarta } \\
\text { (kgha) }\end{array}$ \\
\hline 2,0 & 2313 \\
\hline 3,0 & 5174 \\
\hline 4,3 & 8991 \\
\hline 5,4 & G4 33 \\
\hline 6,9 & 25758 \\
\hline 7,8 & $420=3$ \\
\hline 8,5 & 61990 \\
\hline $9, \theta$ & 80587 \\
\hline 10,6 & 121480 \\
\hline $11, E_{3}$ & 143503 \\
\hline 12,0 & 145057 \\
\hline 13,0 & 148850 \\
\hline 14,6 & 147370 \\
\hline 15,0 & 140436 \\
\hline 16,0 & 145310 \\
\hline 17.6 & 143415 \\
\hline
\end{tabular}

Com o objetivo de estudar a flexibilidade de cada fungfo de ajustar-se as diferentes formas de crescimento foram simulados mal s sete experimentos fletholos $03,4,5,6,7,8,9)$ Cadr um com deressete obser vaçees $(x, y)$, onde $x=a, 3,4, \ldots, 16$ representando a 1 dade, em meses, da reirota da cana-de-acticar, y o peso da planta, em kgha Na Tabela p estalo os dados da lade da plante e os respectivos pesos de massa verde em $\mathrm{kg}$ ha de sethe experimentos. 
TABFLA Paso da cana-de-açucar em kg/ha de massa verde dos Experimentos $3,4,5,0,7,8,9$

\begin{tabular}{|c|c|c|c|c|c|c|c|}
\hline \multirow{2}{*}{$\begin{array}{l}\text { Tdade } \\
\text { Cmeses }\end{array}$} & \multicolumn{3}{|c|}{ Exper i mentos } & \multirow[b]{2}{*}{8} & \multirow[b]{2}{*}{7} & \multirow[b]{2}{*}{8} & \multirow[b]{2}{*}{$\Theta$} \\
\hline & 3 & 4 & 5 & & & & \\
\hline 8 & 2000 & 2000 & 2000 & 2000 & 8000 & 2000 & 2000 \\
\hline 3 & 4000 & 4000 & 4000 & 5000 & 4000 & 8000 & 4000 \\
\hline 4 & 6000 & 7000 & 6000 & 9000 & 7000 & 11000 & 6000 \\
\hline$E S$ & 8000 & 8000 & 8000 & 15000 & 9000 & 18000 & 9000 \\
\hline 63 & 10000 & 13000 & 10000 & 70000 & 12000 & 32000 & 13000 \\
\hline 7 & 13000 & 18000 & 14000 & 40000 & 16000 & 54000 & $\approx 0000$ \\
\hline 8 & 18000 & 25000 & $1 \xi 3000$ & 83000 & 20000 & 80000 & 35000 \\
\hline$\theta$ & 20000 & 41000 & 35000 & 00000 & 34000 & 104000 & 56000 \\
\hline 10 & 30000 & 61000 & 55000 & 117000 & 66000 & 1. 38000 & 85000 \\
\hline 11 & 50000 & 95000 & 100000 & 137000 & 100000 & 146000 & 112000 \\
\hline $1=$ & 101000 & 119000 & 138000 & 148000 & 130000 & 156000 & $1=7000$ \\
\hline 13 & 13000 & 127000 & 144000 & 150000 & 155000 & 168000 & 134000 \\
\hline 14 & 120000 & $1 \geqslant 0000$ & 145000 & 140000 & $10=000$ & 165000 & 137000 \\
\hline 15 & 127000 & 1300000 & 145000 & 149000 & 166000 & 166000 & 140000 \\
\hline 16 & 138000 & 1,29000 & 143000 & 148000 & 166000 & 167000 & $14=000$ \\
\hline 17 & 130000 & 138000 & 142000 & 147000 & 165000 & 166000 & 141000 \\
\hline 18 & 135000 & $1=7000$ & 140000 & 146000 & 184000 & 164000 & 130000 \\
\hline
\end{tabular}

Inicialmente traçaram-se sete curvas diferentes, representadas na Figura 1 que de certa forma, pudessem representar al gumas var açoos no crescimento da planua devido a fatores, tals como diferenca de cultivares, de fertilidade, de dime. Cada curva representando a resposta de um experimento, com as seguintes caractertst, cas:

Os fxpermmentos 3 e s são de balxa produçá, em torno de $130000 \mathrm{~kg} / \mathrm{ha}$.

Os Experimentos E e 6 sfio de produçáo media de 145000 a $150000 \mathrm{~kg} / \mathrm{has}$.

O Fxperimentos $7 \in \varepsilon$ sấ de al ta produçá proximo a $16 r 000 \mathrm{~kg}$ has. 
Frodicios

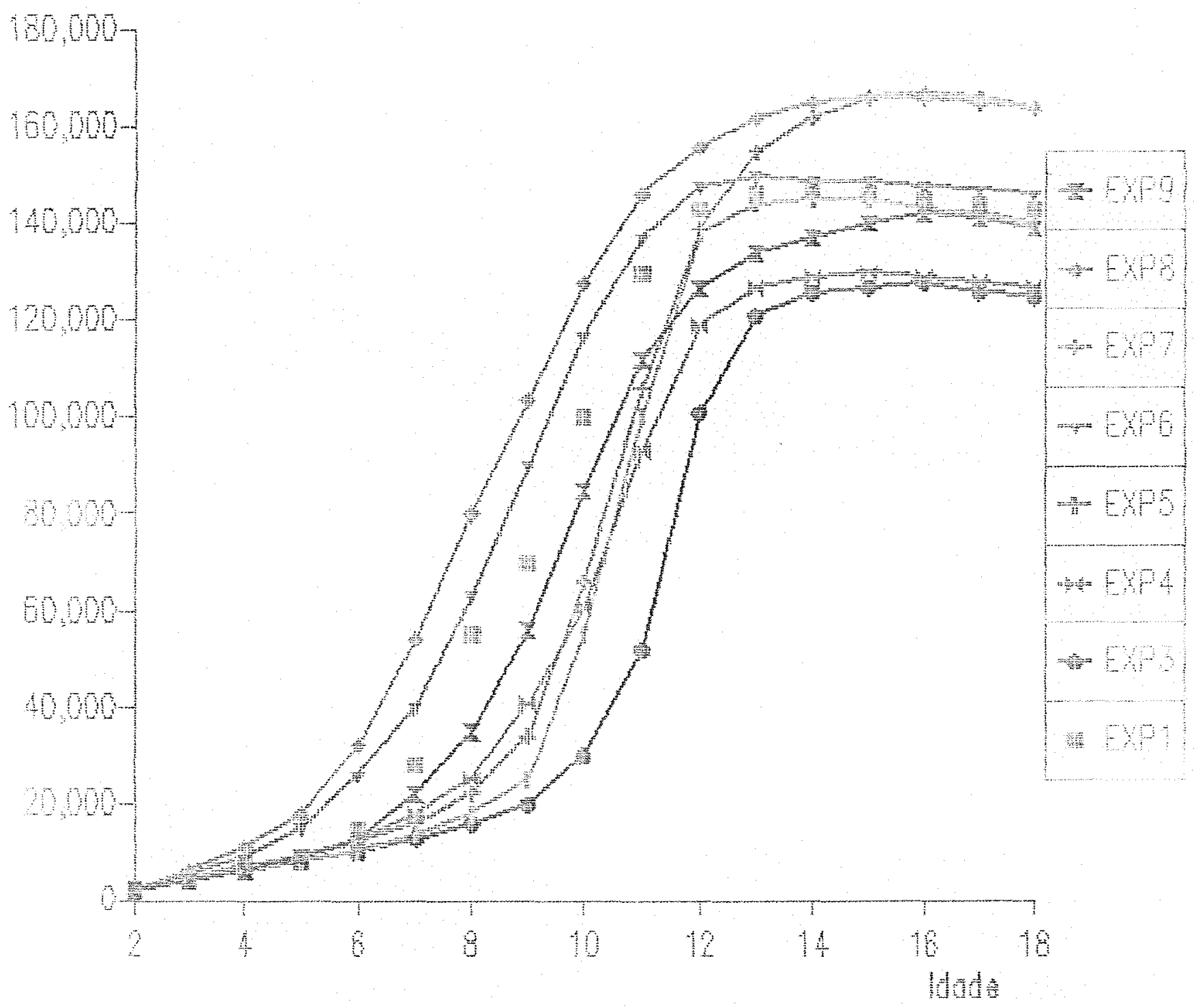

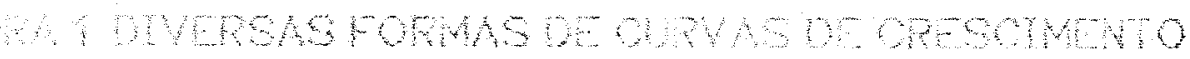


As curvas dos experimentos impares $(3,5$. 73 possuem o ponto de inflexão desiocado para a direita, em relaçá ao seu correspondente experimento par. For exemplo, o ponto de inflexáo do Experimento 3 tem abscissa proxima de 11,0, enquanto que o Experimento 4 tem abscissa proxima de 10,0 . Isto quer diter que os experimentos pares possuem um oreseimento mais suave, di juldo ao 1 ongo do tempo, enquanto que os impares concentram a acelerackio do cresci mento num eurto inter valo de tempo. o Exper i mento $\theta$ Ser i u uma situaçăo intermediarja dos Experimentos $5 \Leftrightarrow 6$, mas atingindo a produça maxi ma apenas no final da curva.

As esti mat vas dos parametros das funsaes. ajustadas com os dados do Exper imento 1 serviram como valores iniciais para o ajuste dos demals experi mentos.

Os valores inicials dos parametros das runcios, quando ajustadas fos dados do Experimento 1 foram tomados conforme cada funcalo, seguindo indicastio de trabal hos anteriores quario existissom, araj jsando a funcrio ou al ria por tentativas. 


\section{Funcoes}

Com base nos graficos e no quadrado medio residual, selecionaram-se doze funçbes, dentre outras, que apresentaram algum nivel de ajuste nos experimentos. Algumas delas foram ldentiffeadas aquj pelo nome do autor da referencia onde for am diadas.

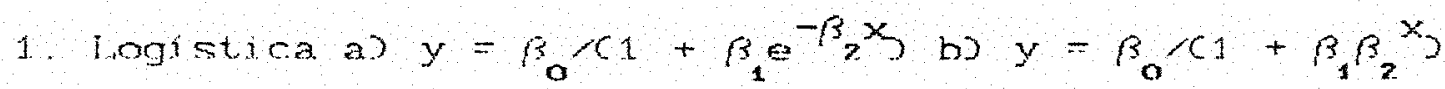

$$
\text { c) } \left.y=\beta_{\alpha} \alpha c+e^{-C \beta_{1}}+\beta_{2}\right)_{3}
$$

$=$ Gompertz d) y $=e^{\left(\beta_{0}-\beta_{1} \beta_{2}\right.} \quad$ el $y=\beta_{0} e^{-\beta_{1} e^{-\beta_{2} x}}$

3. Monomolecular $f$ y $-\beta_{0}\left(1-\beta_{2} e^{-\beta} x_{2}\right.$

4. Brody $\rho$ y $=\beta_{0}-\beta_{1} e^{-\beta} x$

4. logistica Modisicada ho y $=\beta_{0}\left(1+e^{-\beta_{1}} x^{-\beta}\right.$

5. Fxponencial is $\left.y=e^{\left(\beta_{0}-\beta_{2}\right.} \times\right)$

Q. Log-inversa-generailzada jo y $=\beta_{a}+\beta_{1} \beta_{2}$

7. Richards k) y $-\beta_{0} C_{1}+\beta_{0} e^{-\beta} x^{-1 / \beta_{0}}$

e. Re1 1 ) $y=\beta_{0}+\beta_{1} e^{-\beta} x^{\beta}$

Essas funçoes foram comparadas entre si, em Cada experimento, atraves do grafico, do quadrado medio residual COMk, do eriterio de informacko de Akaike CAICS e pelo oof jolente de determinaça ajustado peilo numero de graus de iberande $(\vec{R})$. 


\subsection{Metodo de estimaça}

Fara a estimacra dos parametros foi Lt i l zado o procodi mento NI, IM do SAS "Statietical Anal ysis system", versfio $b$. OE, no qual o usuario deve informar:

- o metodo a ser usado na estimaçá dos parametros.

- a funcfio a ser ajustada.

- As esti mativas lnicials dos parametros.

- as derivadas parciais de $1^{a}$ ordem da funçâo em relaça a cada parametro.

Nầ fol alterado nermum "default" do programa. Para verificar se os valores inicials eram bons para se atingir a ponto de convergeneja, introduziram-se no programa comandos para construcăo do grafico dos valores estimados (x,y), sobreposto ao dos valores observacios $(x, y)$. Todas as est, mat l vas for am calculadas pelo motodo de MAEOUAKNT, sintetizado aqui da seguinte maneira:

sejam Y Variavels aleatoria conhedaks $e x$ varjavel fi xa

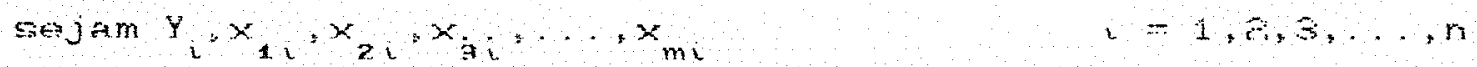
onde $\mathrm{r}=$ numero de observaçes e $\mathrm{m}$ - ntmero de varjaveis ndependerties (Neste caso $\mathrm{m}-1$ )

$F[Y]=f\left(x_{1}, x_{2}, x_{3}, \ldots, x_{m}, \beta_{1}, \beta_{z}, \beta_{3}, \ldots, \beta_{p}\right)+\varepsilon$

$F[Y]=s \in x_{i} / S+\varepsilon$

onde $n^{\times}{ }_{m} \&$ at matrlo de var aveis independentes.

$\beta_{1}$, o vetor dos parametros.

$n^{k_{1}}$ e o vetor dos erros. 
16.

O problema consiste em calcular as estimativas dos parfmetros, minimizando a funça $\Rightarrow \quad \Rightarrow \quad \therefore+y_{i}-\hat{y}_{i} z=\| \hat{y}-\hat{y}_{i}$

onde? $\hat{y}_{i}$ a valor esti mado de $y_{i}$, atraves da expressáa 1.

A funça expandida atraves da serie de

Taylor, fice:

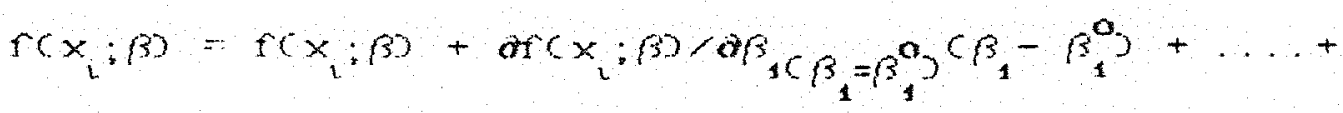

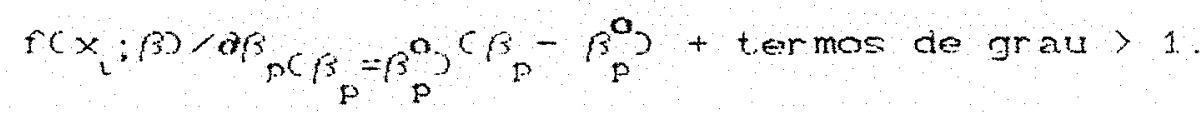

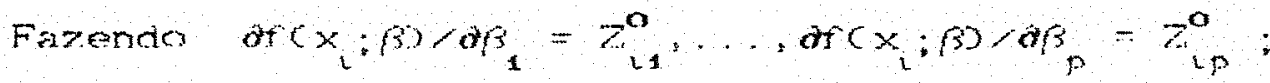

$$
\begin{aligned}
& \beta_{1}-\beta_{1}^{a}=b_{a} \ldots \beta_{p}-\beta_{p}^{o}=b_{p} \\
& f\left(x_{i}, \beta\right)=f_{l}^{\alpha} \text { e desprezando os termos com }
\end{aligned}
$$

expoentes stuperiores a 1 , podemos escrever

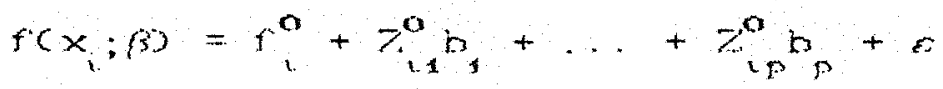

$$
\begin{aligned}
& f c x_{1}, \beta=f_{\nu}^{o}+\sum_{j=1}^{\infty} b_{j}^{o} z_{1}^{o}+c \\
& f\left(x_{L}, \beta\right)-f^{0}=\sum_{J=1}^{p} b_{j}^{0} Z_{L}^{0}+\varepsilon_{2}
\end{aligned}
$$

matricialmente, $\quad y-\mathrm{f}^{\circ}=z^{\circ} \mathrm{h}^{\circ}+\theta$.

onde $b^{\circ}$ aparece como fungào linear.

Felo metodo padrac dos mi ni mos quadrados, obtem-se o sistema de equageres normals CSEN $z^{a} z^{o}=z^{a} c y-f^{o}$, onde $h^{0}$ e soluça de minimos quadrados do sistema o ta tambem a prjmeira estimativa de $\beta . c b^{\alpha}=\beta^{\prime}$ 
Geralmente, a soma de quadrados residual relativa a $b^{\circ}\left(\operatorname{Sok} b^{\circ}\right)$ năo é minima Dá a necessidade de novas itoraços. Fara uma proxima theracáo ha que se fazer uma pequena correcro $C A$ no vetor $b$, na tentativa de diminur a SOE. Fusca-se portanto, $\operatorname{SOKC} \beta^{\mathrm{u}+1},<\operatorname{SQRC} \beta^{\mathrm{u}}, \quad u=1, \tilde{\beta}, 3, \ldots$ Cnumero de jteraçöes.

o problema entao e determi rar a dimensấo a direçấn dex correçăo $A$.

MARQUARDT (1963) sugere 0 valor de $\triangle$ como $\Delta=\left(x^{*} x+\lambda d i a g\left(x^{3} x\right)\right)^{-1} x^{s}$. Na verdade, essa expressão uma integracalo entre 0 metodo de Gauss-Newton, que consider $A=\left(x^{3} \times\right)^{-1} x_{\ell}$ e "Steepest-pescent", ande $A=X^{2}=$

valor $10^{-3}$

Tricialmente, esse autor atribu a $\lambda \quad 0$ Quando $\left.\operatorname{SORC} \hat{\beta}^{\mathrm{u}+1}\right)<\operatorname{SORC} \hat{\beta}^{\mathrm{u}}, \lambda \rightarrow 0$, entado $\lambda$ $\Leftrightarrow$ divido por 10 a diregra do vertor eleterminada peio procedimento do Gauss - Newton.

$$
\text { Se } \left.\operatorname{SoR} \hat{\beta}^{u+1}\right)>\operatorname{SoRC} \hat{\beta}^{\mathrm{u}}, \quad \lambda \rightarrow \infty \text { entao } \lambda
$$
* multipiscado por 10, o o vetor toma a diresáo determi nada por "steepest-Descent". O angulo entre of dois metodos esta, quase sempre, entre $80^{\circ}$ e $90^{\circ}$.

Dessa forma, procura-se aproximar do ponto de minimo da fungro $\$=0$. Natural mente, nato se conhece 6 minimo da funceio \& Em alguns casos, mesmo com as esti matj vas dos parametros proximas do minj mo, o gradjente nể se anula nem por aproximaço, ocasionarido a divergencia do processo iterativo que se torna interminad Del a necessidade do um criterjo pratjoo para a finalizaço do processo, dado pela expressáo 
$\left|\hat{\beta}_{j}^{\mathrm{u}+1}-\hat{\beta}_{j}^{\mathrm{u}}\right|<\delta_{j}$ onde $\hat{\beta}_{j}^{\mathrm{u}}$ e a estimativa do j-esimo componente do vetor $\beta^{\mathrm{U}}$. $O$ autor sugere que os valores de $\delta_{j}$ sejam dados por $\delta_{j}=10^{-4}\left(\beta_{j}+10^{-3}\right.$, onde $10^{-3} \theta$ colocaco para evitar os casos em que $\hat{\beta}_{\mathrm{j}}^{\mathrm{j}}$ são proximos de zero.

Uim ponto importante na estimaçăo de runções não Jineares $a$ escolha dos valores inicials dos par ametros. DRAFFK \& SMITH (1931) sugerem a atribul cáo de valores estimados em trabalhos anteriores, o que nem sempre esta disponf vel. GALLANT (1987) tambem recomenda o uso de jnformaçoses anterioras, a inspecta dos dados, a analish dos orros e clta um metodo geral para estimar os valores rnclats. pependendo da expressáio da furçáo. pode-se ter uma idela do valor aproximado que cada parametro possa assumir, analisancio a expressí́o de acordo com os danns are quals a fumbio dove se ajustar. Tentou-se tambem neste trabalho, adotar 0,1 como valor inicial para todos os parametros, o que náo fol uma boa opeáo na grande maioria dos casos.

0 oriterio do informaço definido por AKATKE $(1974)$ como ATC = NInCSOKJ + EP onde SOR = Soma de quadrados de residuos. $N=$ numero de pontos observadose F - numero de parametros estimados do modelo, permite a comparasio de modelos com diferertes numeros de parametros Guandi as SORs de dols modejos, com diferente numero de parametros, ajustados ao mesmo conjunto de dados săo identicas, o oriterio de informaço apresenta como modelo de melhor ajuste, aquele com menor número de parametros, val dizer o menor valor para AIC. 


\section{RESULTADOS E DISCUSSÖES}

Sao apresentados neste trabalno, os seguintes resultados:

- Est lmatl vas dos parametros

- Aralise de variancla com o seguinte esquens.

$$
\begin{array}{ll}
\text { C. } & G L \\
\text { Fegressao } & F-1 \\
\text { Residuo } & N-F \\
\text { Total } & N-I
\end{array}
$$

- Coeficiente de determinarão $\mathrm{e}^{2}$

- Coeficiente de determinaça corrigido para os 91 dado pela expressa. $\left.P^{2}=F^{2}-C P-10 C 1-R^{2}\right) C N-P$

- O oriterio de informaga de Akalke CAIC $A I C=N 1 \mathrm{~N}(S O P)+2 P$ onde, para todas as expressoes.

$$
\begin{aligned}
& N=\text { rumero de pontos estimados } \\
& P=\text { numero de parametros do modelo. } \\
& S Q F=\text { Soma de quadrados de residuos. }
\end{aligned}
$$


20 .

\subsection{Ajuste das funçóes aos dados do Experimento 1}

\section{Função logistica}

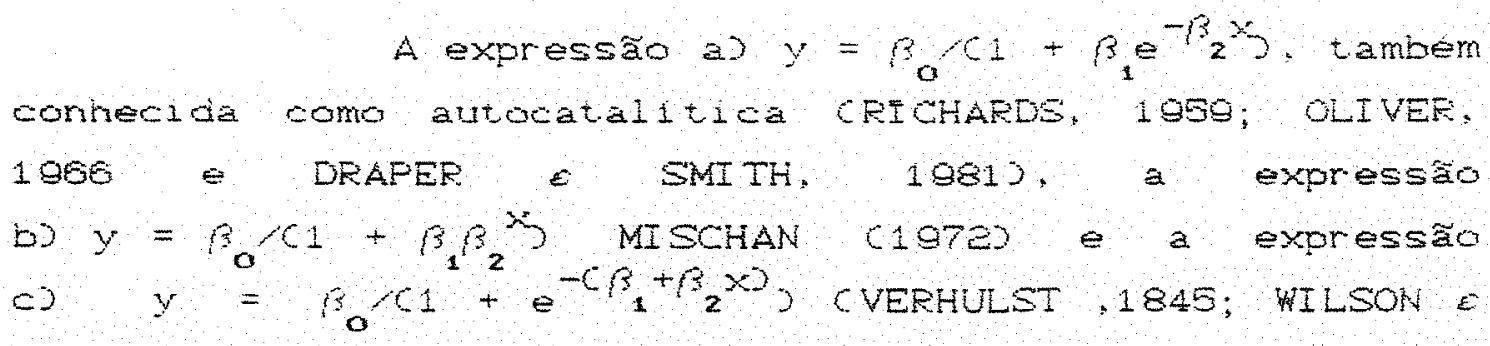
DOUGLAS, 1969 ; VIEIRA, 1975 ;, SILVEIRA, 19760 , ajustaram-se muito bem aos dados, apresentando valores mul to proximos para as somas de quadrados da regressac.

Essas fumcoes, ras formas $\mathrm{Ca}$ e e Cb tem a vantagem de não exigir multo dos valores inicials para que a convergencia seja alcançada. RICHARDS C1959, estudando a funcão logistica na forma las fatuocataliticaj concluiu que $\beta_{0} e$ vaior da assiritota superior $\beta_{1}$ e o valor de y no tempo $x=0, e f_{2}=0$ desiocamento da curva com dase restas iniormacbes, atriburam-se abs parametros os seguntes valores iniciais: $\beta_{\alpha}=150000, \beta_{1}=1500$, $\mathrm{B}_{2}-\mathrm{o}, \mathrm{s}$ Anda for am experimentados valores bastante dispersos para fo cvalores alos e. mesmo assim a convergencia fol atingida.

Outra vantagem da furça cad e que, mesmo partinco de valores diferentes para as estimativas inucials, a convergencia quase sempre acontece e, restes casos, atingiu sempre o mesmo ponto.

Aplicando o logarltmo as expressos Ca (b) e Coj, observamos que as estimativas do parametro $b_{0}$ sá 1 guals nos tres casos. Para o parametro $\beta_{1}$ aperas

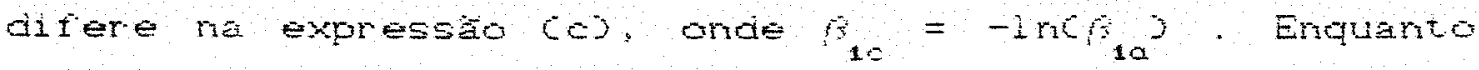
que $r_{2}$ difere da estimativa obtida em lad aperas na 
e1.

expressao (b), onde $\beta_{2 b}=e^{-\beta} 2 a$. Usaram-se estes valores corrigidos, como valores iniciais para ajustar as expressoes $\mathrm{Cb}$ e Coj aos dados do Experimento 1. Tentaram-se outros valores inicials, e o que se pode observar fol que em (a) e cb) a convergencia ocorreu com malor frequencla e sempre para o mesmo ponto, embora em Caj ela tenha ocorrido com malor rapidez do que em Cbo.

$$
\text { A expressão } \quad \text { a } y=\beta_{0} \gamma_{1}+e^{-c \beta_{1}+\beta_{2} \gamma_{j}}
$$

CVERHULST, 1845 ; HILSON E DOUGLAS, 1969 ; VIETRA, 1975 e SILVEIFA, 1976 tambem apresenta uma convergencia bastante rapida, porem exige valores inlcials multo proximos do porto de minimo da funçăo 3 . For exempio, admitindo como valores inicials, os mesmos usados para o ajuste da funçăo autocatalitica, corrigindo $\beta_{10}=-\operatorname{lnc} \beta_{1 a}$, ou seja. $\beta_{1}=-7,301$, a convergencla e raplda e atinge o mesmo porto de minimo, que a primeira fungáo. Quando se atribuem outros valores inicials para os parametros, por exemplo $\beta_{0}=150000, \sigma_{1}=5 ; \gamma_{2}=0,1$, converge rapidamente, mas as estimat 1 vas dos parametros obtldas aqui hä foram 3s mesmas que as anteriores $c_{0}=139137$; $\hat{\beta}_{1}=-18,423 ; \hat{\beta}_{2}=2.09 \quad$ SQResiduo $=3.2 \times 10^{\circ}$.

Realimentando o processo, usando estas estimativas como valores inicials na tentativa de diminuir a SQResiduo, ná se consegui u neninuma melhora. o processo converge para um ponto proxi mo do mim mo e por este metodo de estimação não se consegue aproxima-10 alnaa mals do min mo absoluto. VIEIRA C19753 usou a metodo de NELDER C1961), para estimar os parametros desta funfá obtendo bons resultados os resultados obtidos com o ajuste da fungad loglstica nas formas a, b, e cestao nos quadros de numero $1 \mathrm{a}$. 
QUADRO 1 -

Ajuste da Função Logistica cas

$y=\beta_{0} C_{1}+\beta_{1} e^{-\beta_{2}} x_{2}$ aos Dados

do experi nerto 1

\begin{tabular}{|c|c|c|c|c|c|}
\hline & $\beta_{0}$ & $\beta_{1}$ & $\beta_{2}$ & QMP & $u^{3}$ \\
\hline Valores iniciais & 150000 & 1500,000 & 0,500 & & \\
\hline Estimativas & 149293 & 1573,307 & 0,820 & $3.208 \times 10^{\circ}$ & 12 \\
\hline
\end{tabular}

1 Para todos os quadros, $\mathrm{u}=$ numero de iteraçóes

QUADRO 2 - AJuste da Funça Logistica

Cbo $y=\beta_{0} C_{1}+\beta_{1} \beta_{2}$ aos Dados

do Experimerito 1

\begin{tabular}{llllll}
\hline & $\beta_{0}$ & $\beta_{1}$ & $\beta_{2}$ & QMP & u \\
\hline Valoresthicials & 150000 & 1500,000 & 0,600 & & \\
Estimativas & 149094 & 1572,753 & 0,441 & $3,208 \times 10^{7}$ & 74 \\
\hline
\end{tabular}

QUADRO 3 - Analise de Varlarela das Fumebes Logisticas Ca e Cb Ajustadas ase Dados do Experimerto 1

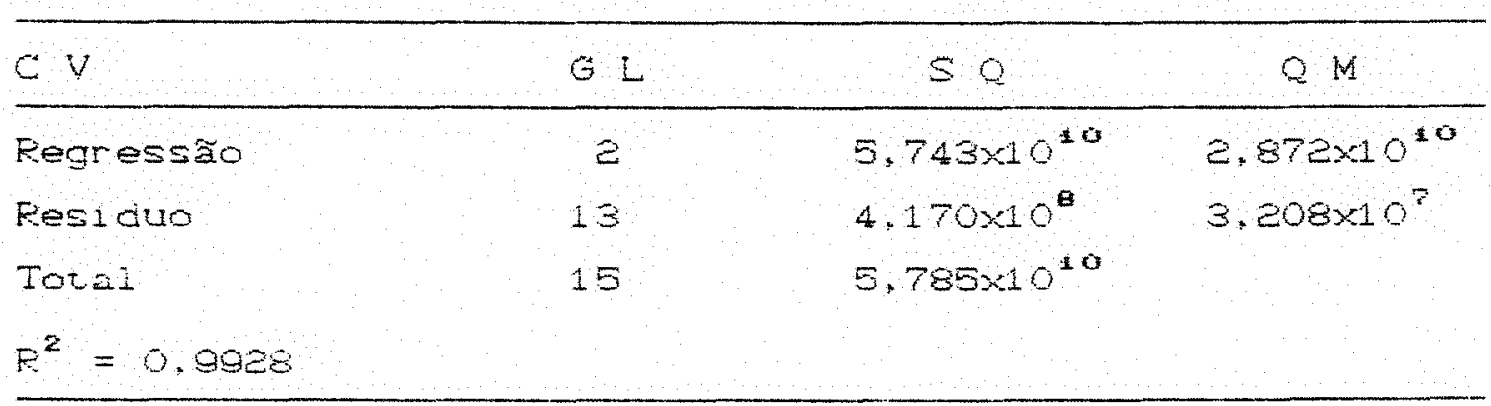


QUADRo 4 - Ajuste da Função Logistica Co

$\left.y-\beta_{0} c_{1}+e^{-C \beta_{1}}+\beta_{2}\right)_{3}$ aos Dados

do Experimento 1

\begin{tabular}{lccccc}
\hline & $\beta_{0}$ & $\beta_{1}$ & $\beta_{2}$ & OMR & 4 \\
\hline Valores iniciais & 150000 & $-7,361$ & 0,838 & & \\
Estimativas & 149216 & $-7,400$ & 0,833 & $3,200 \times 10^{7}$ & 10 \\
\hline
\end{tabular}

QUADRO 5 - Analise de Variancia da Funcão Logistica lo Ajustada aos Dados do Experimento 1

\begin{tabular}{lccc}
\hline Cv & $G L$ & 50 & $0 \mathrm{M}$ \\
\hline Regressar & 2 & $5,743 \times 10^{10}$ & $2,871 \times 10^{10}$ \\
Residuo & 13 & $4,172 \times 10^{\circ}$ & $3,259 \times 10^{7}$ \\
Total & 15 & $5,785 \times 10^{10}$ & \\
$R^{2}=0,9927$ & & & \\
\hline
\end{tabular}

Fumca de Gompertz

A tumça de Gompertz, tambem conherida como complemerto $109-10 g$ e mals usada para representar curvas de erescimento animal do que vegetai una caracteristica interessante desta função $€$ a assimetria da curva em relaça ao ponto de inflexão. VIEIRA (1975) estudou a funcao cd $y-e^{C \beta}{ }_{0}^{-\beta} \beta_{2}{ }_{2}$ para representar o crescimento de ratos, usando o metodo de STEVENS 41951 ) para estamar os parametros. A curva de forma sigmolde apresenta duas assintotas, $y=0$ cinferior $e y=e^{\beta}$ o csuperiors.

0 ponto de inflexá e dado por $x=-1 n \rho_{1} / h_{2} e$ $y=e^{C \beta^{-1}}$. Da assintota superior pode-se deduzir $a$ 
valor inicial para $\beta_{0}$ seja ycmaximo o maior valor observado do peso da planta. Considerando ycmaximo como assintota superior, tem-se que $\operatorname{lrcycmaximos}=\beta_{0}$ logo 0

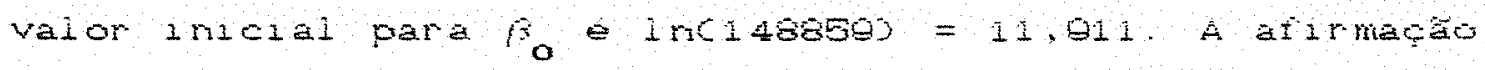
da autora e que $\beta_{1} 0$ e $\beta_{2} \beta_{2}$ Neste trabalio consideraram-se os valores 2 nidals $r_{0}=11,911, \beta_{1}=10$, $\mathrm{B}_{2}=0.5$, para estimar os parametros da funça $\mathrm{Cd}$, ajustada aos dados do Experimento 1 .

Outra funçăo de Gompertz fol estudada por RICHARDS $61059, \mathrm{C}=\mathrm{y}=\beta_{0} e^{-\beta_{1} e^{-\beta} \mathrm{\beta}_{2}}$, cujo ponto de

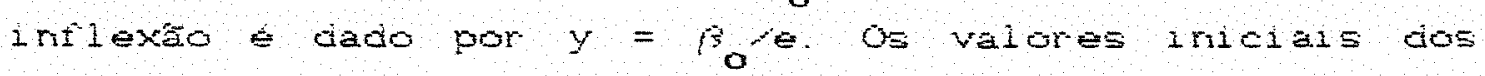
parametros para o ajuste desta furga podem ser deduzidos da fumpao anterior. Apl cando o logaritmo duas vezes em ambas as funcoes tem-se.

$$
\begin{aligned}
& -E m(d), \quad \ln C y=\beta_{0}-\beta_{1} \beta_{2} \\
& \operatorname{lnc} \beta_{0}-1 \mathrm{ncy}=\operatorname{lnc} \beta_{1}+x \operatorname{lnc} \beta_{2}
\end{aligned}
$$

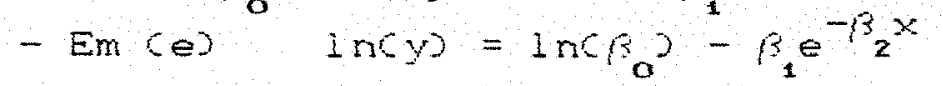

$$
\begin{aligned}
& \operatorname{lnClnC\beta } \beta_{0}-\operatorname{InCy}=\operatorname{InC} \beta_{1}-x \beta_{2}
\end{aligned}
$$

Logo, os valores inicials para a expressa cej săo: $\beta_{0}=e^{11, \phi_{1}}=148895, \beta_{1}=10, \beta_{2}=-1 n(0,5)=0,693$.

Ambas as funcoes estudadas aqui apresentaram un bom ajuste na malor 1 a dos experimentos. at ingindo sempre o mesmo ponto de minlmo de $s$, en poucas iterafoes.

Mostraram tambem năo ser muto exigentes quanto aos valores lnidials dos parametros, o que e uma caracteristica desejavel, pois rem sempre o usuario tem informacoes sobre os valores iniciais dos parametros os resultados obtidos com o ajuste da funçao de Gompertz, ras formas $\mathrm{C} d \mathrm{CO}$ estä nos quadros $\theta, 7 \& \theta$. 
QUADRo 6 - Ajuste da Funça de Gompertz cd

25 $y=e_{0}-\beta_{1} \beta_{2}^{x}$ aos Dados do Experimento 1

\begin{tabular}{|c|c|c|c|c|c|}
\hline & $\beta_{0}$ & $\beta_{1}$ & $\beta_{2}$ & $\mathrm{QME}$ & $u$ \\
\hline Valores iriciais & 11,911 & 10 & 0.500 & & \\
\hline Estimativas & 11,928 & 110,196 & 0.565 & $6.275 \times 10^{7}$ & 16 \\
\hline
\end{tabular}

QUADRO 7 - AJuste da Funça de Gompertz 60 $y=\beta_{0} e_{1}^{-\beta_{1} e^{-\beta}{ }^{x}}$ aos Dados do Exper 1 mento 1

\begin{tabular}{|c|c|c|c|c|}
\hline & $\hat{\theta}_{0}$ & $\beta_{1}$ & $\theta_{2}$ & $\mathrm{OME}$ \\
\hline Valores inicials & 148805 & 10 & 0.093 & \\
\hline Estimativas & $15141 \mathrm{e}$ & 110,190 & 0,571 & $0.275 \times 10^{7}$ \\
\hline
\end{tabular}

QUADRo 8 - Analise de Varlancla das funços de Gompertz (d), (e) Ajustadas aos Dados do Experimento 1

\begin{tabular}{llll}
\hline CV & GL & 50 & om \\
\hline Regressao & 2 & $5.703 \times 10^{10}$ & $2.852 \times 10^{10}$ \\
Residuo & 13 & $8,158 \times 10^{\circ}$ & $5.275 \times 10^{7}$ \\
Tota1 & 15 & $5,785 \times 10^{10}$ & \\
$\mathrm{R}^{2}=0,9858$ & & & \\
\hline
\end{tabular}




\title{
Funç̋̃o moromolecular
}

\begin{abstract}
$A$ expressão co $y=\beta_{0} C_{1}-\beta_{1} e^{-\beta_{2}} x_{e} e$ tambem conhecida cono funça monomolecuiar. RICHAEDS 61959 dita-a como uma das tres funcres mals usadas para 0 ajuste de curvas de crescimento ao lado das funças autocatalitica $\mathrm{Ca}$ e de Gompertz $\mathrm{Cd}$ ).
\end{abstract}

Neste trabalho, estudou-se tambem a funcão Brody $\mathrm{cg} y=\beta_{0}-\beta_{1} e^{-\beta_{2} \alpha}$, onde $\beta_{19}=\beta_{\text {of }} \beta_{1 f}$ da funça anterior, GALLAN (1887) e SILVEIRA C1976) Ambas as funçses naturalmente apresentaram o mesmo ajuste. Mostraram năo ser muto exigentes quanto a precisăo dos valores inicials. Convergiu com relativa rapidez, quardo ajustadas aos dados do Experimento 1 .

Embora a funça Cf tenha sido cltada por PICHARDS 1959 como uma das tres mals usadas no ajuste de dados de orescimento, neste trabalho, näo demonstrou ser uma das mel hores, quando comparada as demais.

\footnotetext{
Como valores inicials foram usados

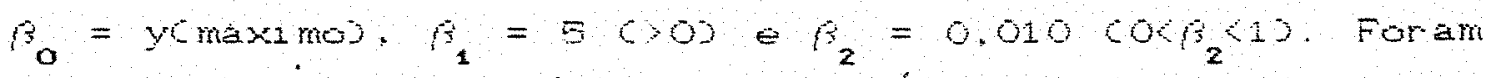
testadas varias opcoes como valores iniciais para verificar se as estimativas encontradas eram de aigum minimo local, dado que a função näo se ajustou bem a esse conjunto de dados. No entanto, todas as vezes en que a convergencla sol encontrada, ela se deu sempre no mesmo ponto. fazendo crer que este seja o ponto de minimo globail, da funçăo $\$$. os resultados do ajuste das funç̧es monomolecular e de Erody estan nos quadros $9,10, e 11$.
} 
QUADRO 9 - Ajuste da Funcro Monomolecular Cf $y=\beta_{0}\left(1-\beta_{1} e^{-\beta} x_{2}\right.$ aos Dados $d o$ Experimento 1

\begin{tabular}{lcccc}
\hline & $\beta_{0}$ & $\beta_{1}$ & $\beta_{2}$ & MR \\
\hline Valorestinicials & 148859 & 5 & 0,010 & \\
Estimativas & 362593 & 1,151 & 0,043 & $4,651 \times 10^{\circ}$ \\
\hline
\end{tabular}

QUADRO 10 - Ajuste da funcão Brody $\cos$ y $=\beta_{0}-\beta_{1} e^{-\beta_{2}} x$ aos Dados do Exper 1 mento 1

\begin{tabular}{lllll}
\hline Valores inicials & 365086 & 420500 & 0,040 \\
Estimativas & 360550 & 417354 & 0,043 & $4,651 \times 10^{6}$
\end{tabular}

QUADRO 11 - Analise de Variancia das funçóes (f) e (g) Ajustadas aos Dados do Experimento 1

\begin{tabular}{lccc}
\hline cV & GL & So & QM \\
\hline Regresså & $e$ & $5,180 \times 10^{10}$ & $0,590 \times 10^{10}$ \\
Residuo & 13 & $6,046 \times 10^{\circ}$ & $4,651 \times 10^{\circ}$ \\
Total & 15 & $5,785 \times 10^{10}$ & \\
$R^{2}=0,8954$ & & & \\
\hline
\end{tabular}


28.

\section{Funça logistica modificada}

A função $\left.c_{b}\right) y-\beta_{0}\left(1+e^{-\beta_{1}} x^{-\beta_{2}}\right.$ fol usada por CORTARFIAI (1973), para ajustar a dados de crescimento de gado bovino. No presente trabal ho, o modelo apresentou um hom ajuste na maj or ia dos experimentos.

Nâo se tinha informaço mais precisa sobre os valores iniciais dos parametros. For esta razao, opton-se por atribut a $\beta_{\alpha}$ um valor proximo do yomaximo a $\beta_{1}$ \& $\beta_{2}$ vajores bajxos, por apresentarem-se na expressä, como expontes:

Năo o uma funcaro muito exigente quanto a precisăo dos valores iniclais. Fode-se notar no Quadro 1 a que esolthe do valor injoial para $\beta_{2}$ năo fol muto adequada No ontanto a convergenoia fol atingida. Experimentando outros val ores inioials para os parsmetros, toda vez que a convergencia fol encontrada, ala se deu atingindo-se o mesmo ponto de minimo, apesar de ds vezes ser extremamente lento, o processo de convergenela.

QUADKO 12 -- Ajuste da Função logistica Modílicada

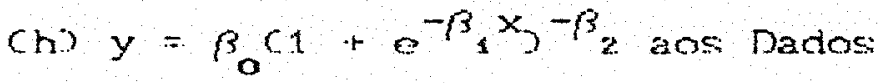
do Fixperi mento 1

\begin{tabular}{|c|c|c|c|c|c|}
\hline & $\beta_{0}$ & $\beta_{1}$ & $\beta_{2}$ & $\mathrm{OMR}$ & u \\
\hline Valores juleials & 148858 & 0,100 & 0,010 & & \\
\hline Est, mativas & 1.51970 & 0,555 & 96,498 & $6,866 \times 10^{7}$ & 67 \\
\hline
\end{tabular}


20 .

QUADRO 13 - Analise da Variancia da Funçao Logistica Modi ficada Ajustada aos Dados do Experimento 1

\begin{tabular}{lccc}
\hline CV & GL & So & OM \\
\hline Regresså & 2 & $5,704 \times 10^{10}$ & $2,852 \times 10^{10}$ \\
Residuo & 13 & $8,146 \times 10^{\circ}$ & $6,266 \times 10^{7}$ \\
Total & 15 & $5,785 \times 10^{10}$ & \\
$R^{2}=0,9860$ & & & \\
\hline
\end{tabular}

\section{Funçæo exponencial}

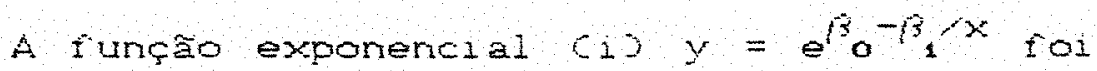
citada por ARPUDA et all (1973), a partir da qual os autores suger 1 ram a furç̋̈o log-inversa-generalizada, que tamben fol assunto deste trabaino, para ajustar a dados de crescimento.

A função exponencial não apresentou um dos melhores ajustes. Uma vantagem desta função e a convergencia multo rapida do processo a partir de valores iniclais não multo precisos. Fol uma das poucas funços que atirgiram a convergencia a partir do valor 0,1 atribuido aos parametros, em apenas 14 lteraços.

A indicaça para os valores inicials dos parametros e de In y maximo para $\beta_{0} e \beta_{1} 0$. Para o ajuste aos dados do Experimento 1 , atribuiu-se a $\beta_{0} 0$ valor 11.911 e a $\rho_{1}$ o valor 10 os resultados encontram-se nos quadros 14 e 15 
QUADRO 14 - Ajuste da Funça Exponencial (i) $y=e_{0}-\beta_{1} x$ aos Dados do Experimento 1

\begin{tabular}{lllll}
\hline & $\beta_{0}$ & $\beta_{1}$ & OMR & 4 \\
\hline Valorestriciais & 11,811 & 10 & & \\
Estimativas & 12,867 & 14,529 & $3,298 \times 10^{8}$ & 7 \\
\hline
\end{tabular}

QUADRo 15 - Anal 1 se de Variancia da Função Exponencial Ajustada aos Dados do Experimento 1

\begin{tabular}{lccc}
\hline CV & GL & SQ & oM \\
\hline Regressán & 1 & $5,323 \times 10^{10}$ & $5,323 \times 10^{10}$ \\
Residuo & 14 & $4,617 \times 10^{\circ}$ & $3,298 \times 10^{\circ}$ \\
Total & 15 & $5,785 \times 10^{10}$ & \\
$R^{2}=0,920 \mathrm{C}$ & & & \\
\hline
\end{tabular}

\section{Funça log-inversa-generalizada}

Esta funça fol usada por AREUDA et dlli C1973s para descrever curvas de oreselmento da altura de Pinus caribed e cresolmento ponderal de gado boviro os autores sugeriram o uso desta funcăo en estudos de ajuste a dados de crescimento, al em das mals conhecidas, como logistica e de Gompertz. Esta funcso tamben fol usada por VEIRA 61975 s para descrever o crescimento de ratos.

$$
\begin{aligned}
& \text { A Iunça log-inversa-generalizada cjo } \\
& y=\beta_{0}+\beta_{1} \beta_{2}^{1} \text { para } \beta_{1} 0 \text { e } \alpha \beta_{2}<\text { apresenta ponto de } \\
& \text { inflexá } x=-\ln \beta_{2} \rho e y=\beta_{0}+\beta_{1} / e^{2} \text {, tendo como } \\
& \text { assintota superior } y=\beta_{0}+\beta_{1} \text { o valor de } \beta_{0} \text { edado como }
\end{aligned}
$$


sendo 0 valor de y para $x=0$. Nostra tambem näo ser muito exigente quarto a escolna dos valores imicials embora deperidendo dos valores inicials adotados a convergencia se torna muito ienta. Os resultados estão nos quadros $16 e$ 17 .

QUADRO 16 - Ajuste da Funçăo Log-inversa-generalizada $C j$ $y=\beta_{0}+\beta_{1} \beta_{2}^{1 x}$ aos Dados do Experimento 1

\begin{tabular}{|c|c|c|c|c|c|}
\hline & $\beta_{0}$ & $\beta_{1}$ & $\beta_{2}$ & $\mathrm{OME}$ & $\mathrm{u}$ \\
\hline Valores indcials & 1500 & 10 & 0.500 & & \\
\hline Estimativas & -7314 & 378452 & $1,438 \times 10^{-6}$ & $3.454 \times 10^{9}$ & 49 \\
\hline
\end{tabular}

QUADRO 17 - Análise de Variancia da Função Log-inversa-generalizada Ajustada aos Dados do Experi mento 1

\begin{tabular}{lccc}
\hline CV & GL & SQ & QM \\
\hline Regresså & 2 & $5,330 \times 10^{10}$ & $2,608 \times 10^{10}$ \\
Residuo & 13 & $4,490 \times 10^{\circ}$ & $3.454 \times 10^{6}$ \\
Total & 15 & $5,785 \times 10^{10}$ & \\
$P^{2}=0,9224$ & & & \\
\hline
\end{tabular}

\section{Função de Richards}

A função fol estudada por RICHARDS 61959 e CAUSTON C1969, usando a expressä Cks $y=\beta_{0} C_{1} \pm \beta_{1} e_{2} y^{-1}$ r onde o sinal dentro do parenteses segue o sinal de $\mathrm{n}$ o valor de $\mathrm{n}$ de acordo com CAUSTON C1969s deve variar de $C-1 \leq n<0)<0<n$ ná serido definida para $n<-1$ e para $n=0$. Quando $n--1$ tem-se a 
funça monomolecular. Neste trabalho, n fol considerado como o quarto parametro.

0 ajuste desta função fol melhor que o de todas as demais. Como valores inicials dos parametros foram usados $\beta_{0}=150000, \beta_{1}=1000000, \beta_{2}=1, \beta_{3}=1$.

Uma caracteristica muito desejavel em funçoses náo lineares e a capacidade de atingir o ponto de convergencia, a partir de valores inicials ná multo precisos. Como se pode observar, no Quadro 18 a a estimativa de $\beta_{1}$ fol muito diferente do valor inicial. Foram experimentados varios outros valores iriclals para os parametros, entre eles, $\beta_{0}=15000, \beta_{1}=\beta_{2}=\beta_{3}=1$ atinglndo-se a convergencia semore no mesmo ponto.

Sem querer generallzar, pode-se dizer, com base neste trabalho, que na falta de informaçóes mals precisas sobre os valores inicials dos parametros, pode-se atribuir a $\beta_{0}$ o valor de ycmaximo, para $\beta_{1}$ um valor alto, $C \beta_{1}=1,0 \times 10^{\circ}$ tanto mals alto quanto maior for a iriclinaça da curva. para $\beta_{2}$ e $\beta_{3}$ pode-se atribuir 0 valor 1 a ambos os parametros.

Fara a funça ajustada desta forma, com quatro pararietros, quardo se atribul valor negativo para $\beta_{3}$ o ajuste apresenta os mesmos valores absolutos estimados para $\beta_{3}>$ apenas com os sinals trocados para as estimativas de $\beta_{1}$ e $\beta_{3}$. Isto contrarla a definifádo intervalo atribuldo aos valores de $\beta_{3} b-1 \leq \beta_{3}<0.0$ resultados se encontram ros quadros $18 \Leftrightarrow 19$. 
QUADRO 18 - Ajuste da Funçäo de Fichards $(k)$ $y=\beta_{0}\left(1+\beta_{1} e^{-\beta} z\right)^{-1 / \beta}$ a as Dados do Experimento 1

\begin{tabular}{lccccc}
\hline & $\beta_{0}$ & $\beta_{1}$ & $\beta_{2}$ & $\beta_{3}$ & QMR
\end{tabular}

QUADRO 19 - Analise de Variancia da Função de Richards Ajustada aos Dados do Experimento 1

\begin{tabular}{lccc}
\hline CV & GL & So & OM \\
\hline Regressá & 3 & $5,771 \times 10^{10}$ & $1,924 \times 10^{10}$ \\
Residuo & 12 & $1,383 \times 10^{8}$ & $1,153 \times 10^{7}$ \\
Total & 15 & $5,785 \times 10^{10}$ & \\
$R^{2}=0,9970$ & & & \\
\hline
\end{tabular}

\section{Função de keid}

A tunça de Reld, dado pela expressão Cl $y=\beta_{0}+\beta_{1} e^{-\beta} \alpha^{\beta}$, fol usada por SPAFRo C19790, para se ajustar a dados de produço de materia seca de forragem. em funça de nitrogenio. No presente trabalno o ajuste da fungăo de Reld não fol bom.

A função exige que os valores inicials dos parametros sejam proximos das estimativas, que representam 0 ponto de minimo da funca $b$ Conforme os valores inicials, a convergencia se da em diferentes pontos.

A escolna dos valores inicials dos parametros para ajustar a funcáo aos dados do Experimento 1 fol felta por 
tentativas. Optou-se pelas estimativas que apresentaram menor SOR. Naturalmente que, ate atingir as estimativas que foram usadas como valores iniclais, processaram-se muitas lteraçbes. Os resultados sáo apresentados nos quadros $20=21$.

QuAvko 2o - Ajuste da Funçäo de Reid CID

$$
\begin{aligned}
& y=\beta_{0}+\beta_{1} e^{-\beta_{2} \beta_{3}} \text { aos Dados } \\
& \text { do Experimento } 1
\end{aligned}
$$

\begin{tabular}{llll}
\hline & $\beta_{0}$ & $\beta_{1}$ & $\beta_{2} \beta_{3}$
\end{tabular}

QUADRO Z1 - Analise de Variancia da Funçăo de Reid Ajustada aos Dados do Experimento 1

\begin{tabular}{lccc}
\hline CV & GL & So & QM \\
\hline Regressao & 3 & $5,138 \times 10^{10}$ & $1,713 \times 10^{10}$ \\
Residuo & 12 & $8,468 \times 10^{\circ}$ & $5,388 \times 10^{\circ}$ \\
Tota1 & 15 & $5,785 \times 10^{10}$ & \\
$\mathrm{R}^{2}=0,8882$ & & & \\
\hline
\end{tabular}




\section{A. Ajuste das funcers aos dados do fixporimento 2}

As: estjmativas dos parametros das funches

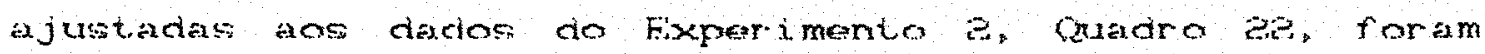
cotidas tencio como valores injojals as estimativas ohtidas con o ajuste das funcones ans dados do Fxperimento 1 .

QUADRO P - Ajugte das Fungres ans Denos do Fxperimento $?$

Fung Estamativas dos parametros OMR $\mathrm{R}^{2} \mathrm{U}$
$\hat{\beta}_{0} \quad \hat{\beta}_{1} \hat{\beta}_{2} \hat{\beta}_{s}$

\begin{tabular}{|c|c|c|c|c|c|c|}
\hline 149870 & 1604 & $0,8=2$ & & $7,334 \times 10^{7}$ & 0.9804 & 15 \\
\hline $14 S P 69$ & $160 \%$ & 0,440 & & $7,234 \times 10^{7}$ & 0,0801 & 103 \\
\hline 148040 & $-6, \Omega O$ & 0,784 & & $7,860 \times 10^{7}$ & 0,0787 & \\
\hline 11.930 & 111 & 0.564 & & $0.680 \times 10^{7}$ & 0.9737 & \\
\hline 151393 & 111 & 0.587 & & $9,6880 \times 10^{7}$ & 0,9787 & \\
\hline 363215 & 1,151 & 0.043 & & $4.275 \times 10^{8}$ & 0,8830 & \\
\hline 363215 & 418008 & 0,043 & & $4.875 \times 10^{8}$ & 0.8830 & \\
\hline $161 \leqslant 7$ & 0,557 & 97 & & $0,681 \times 10^{7}$ & 0,9737 & \\
\hline $1 \mathrm{R}, 8 \mathrm{ES}$ & 14,948 & & & $3,360 \times 10^{8}$ & 0,0084 & \\
\hline-7385 & 378866 & $1.43 \times 10^{-6}$ & & $3,20 \times 10^{8}$ & 0,0100 & \\
\hline $14 E S A 2 S$ & $4,419 \times 10^{11}$ & $P .465$ & 5,884 & $5,5 i 44 \times 10^{7}$ & 0,0981 & 653 \\
\hline-61 Eस? & $1,580 \times 1.0^{-13}$ & $-39,671$ & 0,018 & $4,545 \times 10^{8}$ & 0,8768 & \\
\hline
\end{tabular}


36.

4.3. Ajuste das funcós aos dados dos Experimentos 3, $4,5,6,7,8,9$

Para ajustar as funçós aos dados dos demais experimentos $3,4, \ldots, 9$, cujos resultados estăo nos Quadros 23 a 34 , for am usadas, como valores inielais dos parametros, as estimat, vas obtidas com o ajuste aos dados do Experimento 1. O Quadro 23 mostra os resultados dos ajustes da logistica, na forma $(a)$, aos experimenntos.

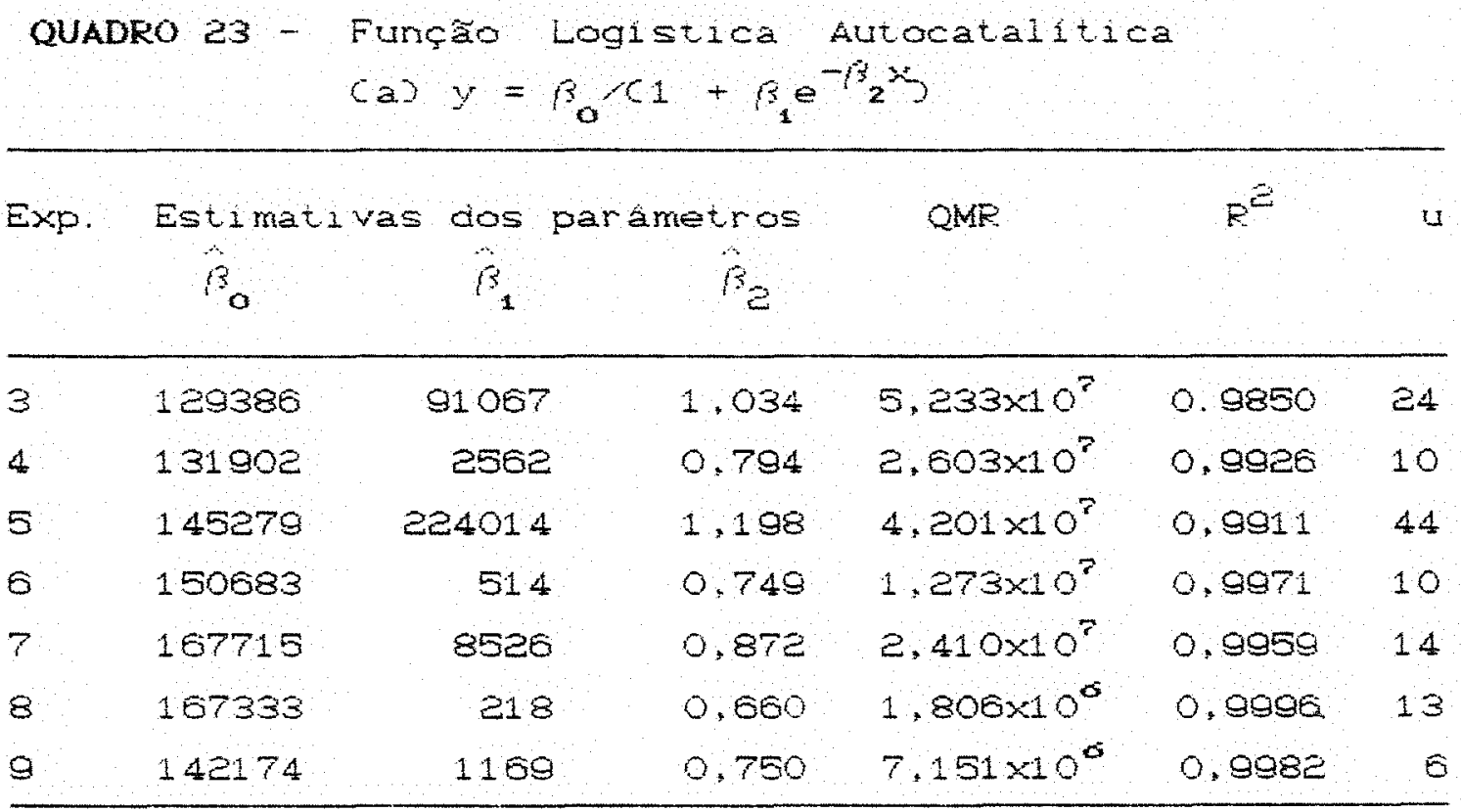

Pode-se observar que os valores de $\hat{\beta}_{0} s a_{0}$ ilgeiramente superiores as produçbes maximas observadas nos respectivos experimentos $\hat{\beta}_{1}$ e a projeça da produça no tempo zero. Observa-se que nos Experimentos $3,5 e 7$, cujo erescimento e mals acelerado, $\hat{\beta}_{1}$ ná corresponde ao ponto de intersefáo da curva ao elxo das ordenadas. For sua vez $\beta_{z}$ determina a inclinação da curva, conforme observou RICHARDS $619593 \hat{\beta}_{2}$ e maior, onde 0 crescimento mais acelerado, vaja os valores de $\hat{\beta}_{2}$ nos Experimento 3. 
5, e 7 , comparados a $4,6, e 8$, respect ivamente.

os Experimentos pares, ros quais o crescimento fol mais lerto, apresentaram melhor ajuste, princlpalmente onde a produça fol malor CExperimento 8), que apresentou menor OMR e maior $\mathrm{R}^{2}$.

A assintota superior e dada por $\hat{\beta}_{0}$ muito proxima das produços maximas em cada experimento.

o ponto de inflexá é dado pela coordenadas $x=\ln \beta_{1} \beta_{2}$ $y=\hat{\beta}_{o}, 2$.

No Quadro 24 encontram-se os resultados dos ajustes da funcão logistica, na forma cb), aos dados dos experimentos.

QUADRO 24 - Funcão Logistlca cbj $y=\beta_{0} C_{1}+\beta_{1} \beta_{2}{ }^{x}$

\begin{tabular}{|c|c|c|c|c|c|c|}
\hline \multirow{3}{*}{$\begin{array}{l}\text { Exp. } \\
3\end{array}$} & \multicolumn{2}{|c|}{ Estimativas dos } & \multirow{2}{*}{$\begin{array}{c}\text { parametros } \\
\qquad \hat{\beta}_{2}\end{array}$} & \multirow{2}{*}{$S Q M P$} & \multirow[t]{2}{*}{$R^{2}$} & \multirow[t]{2}{*}{$u$} \\
\hline & $\beta_{0}$ & $\hat{\beta}_{1}$ & & & & \\
\hline & 129382 & 91449 & 0,355 & $5,232 \times 10^{7}$ & 0,9850 & 612 \\
\hline 4 & 131903 & 2560 & 0,453 & $2,603 \times 10^{7}$ & 0,9920 & 403 \\
\hline 5 & 145277 & 224707 & 0,302 & $4,201 \times 10^{7}$ & 0,9911 & 1408 \\
\hline 6 & 150682 & 514 & 0.473 & $1.273 \times 10^{7}$ & 0,9971 & 103 \\
\hline 7 & 167716 & $852 e$ & 0,418 & $2,410 \times 10^{7}$ & 0,9959 & 519 \\
\hline 8 & 167333 & 218 & 0,517 & $1,806 \times 10^{\circ}$ & 0.9986 & 101 \\
\hline 9 & 142174 & 1169 & 0,472 & $7,151 \times 10^{6}$ & 0,9982 & 192 \\
\hline
\end{tabular}

A única diferença com relação a função autocatalitica esta em $\hat{\beta}_{2}$, que e menor onde o crescimento e mals acelerado. A assintota super or e dada por $\hat{\beta}_{0}$ tambem 1 lgeiramente superior as produçoses maximas de cada experimento. 0 ponto de inflexao e dado pelas coordenadas $x=-i n \beta_{1} I_{2}, \quad y=\beta_{0} \beta_{2}$ As figuras $2,3,4$ e mostram o ajuste das funços logisticas ras formas $l a s \in C b j$. 


\section{EXPERIMENTO 1}

Fasteritis

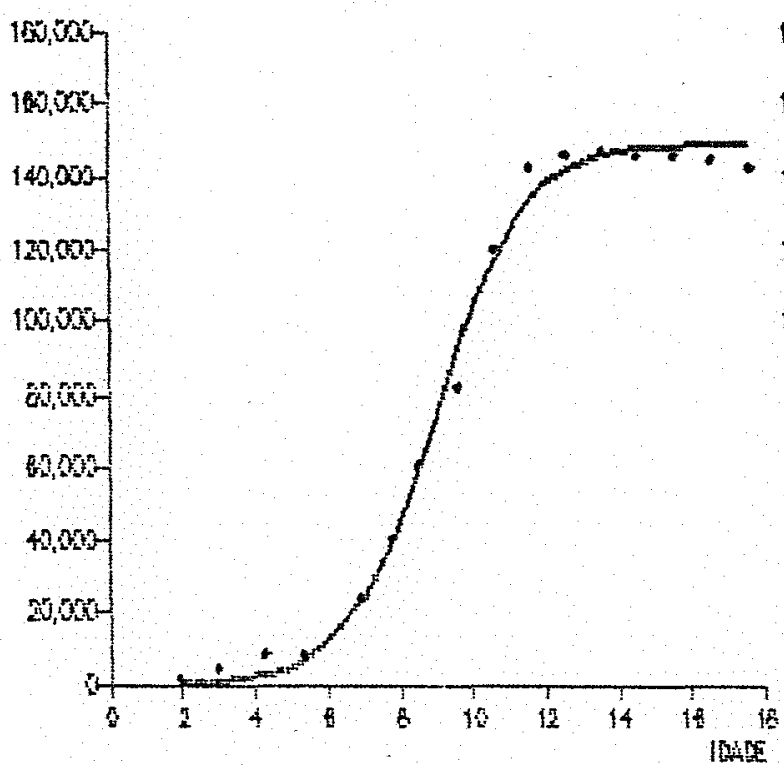

EXPERIMENTO 3

Ponsulatin

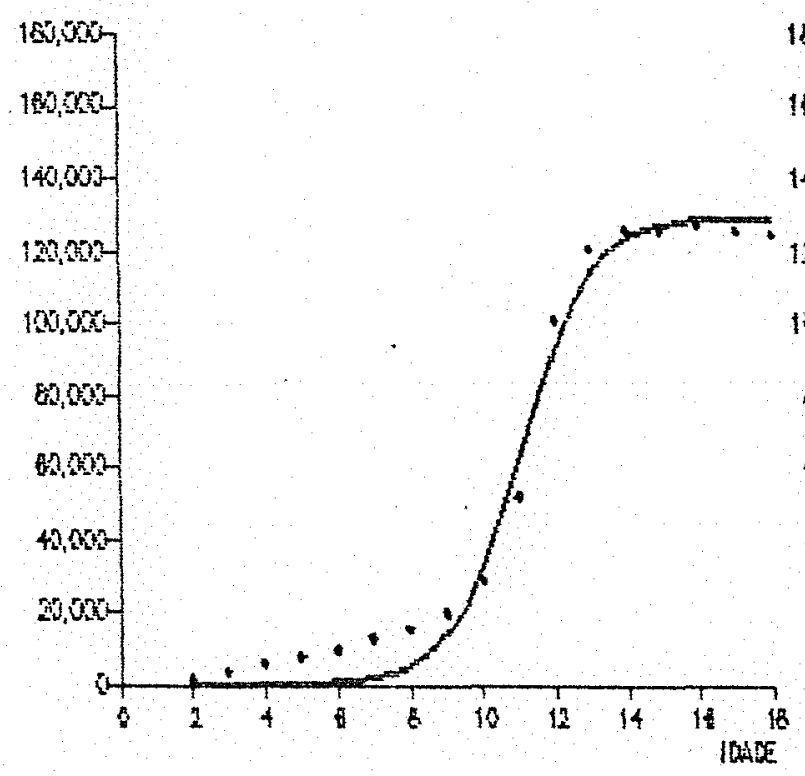

EXPERIMENTO 9

maxing

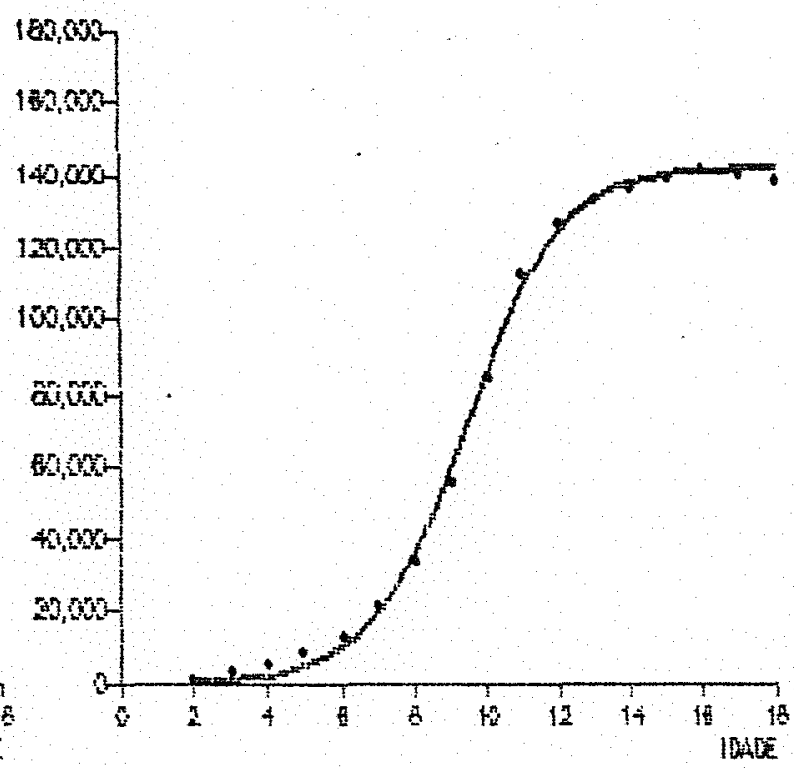

Finin

EXPERIMENTO 4

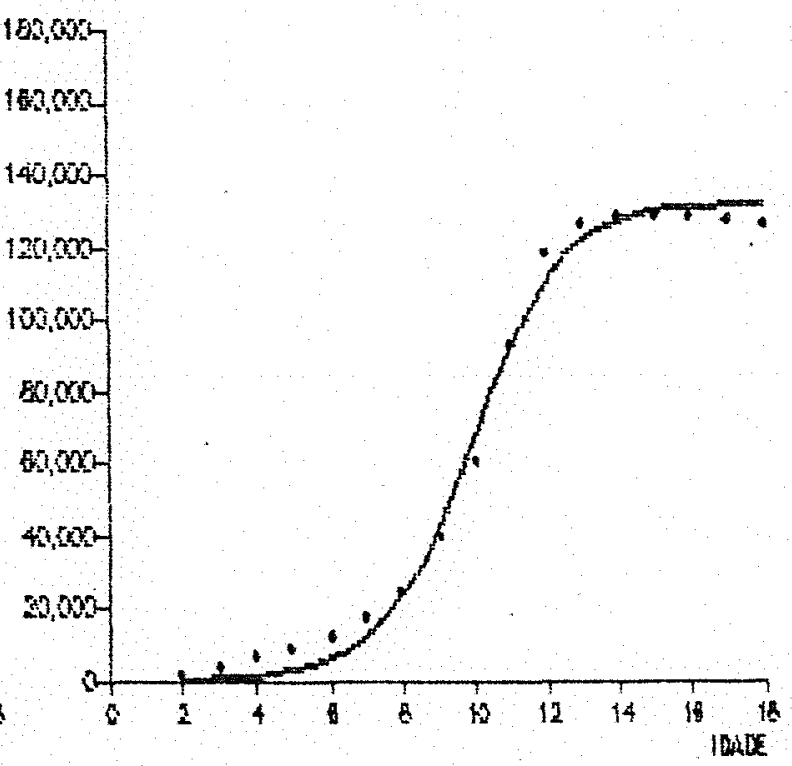

Figura a - Graficos das curvas ostimadas atraves da rurifto logs sitica (a) robropostos aos pontos otserervados ries Exper i mentess $1,9,3,4$. 
EXPERIMENTO 5

proxuta

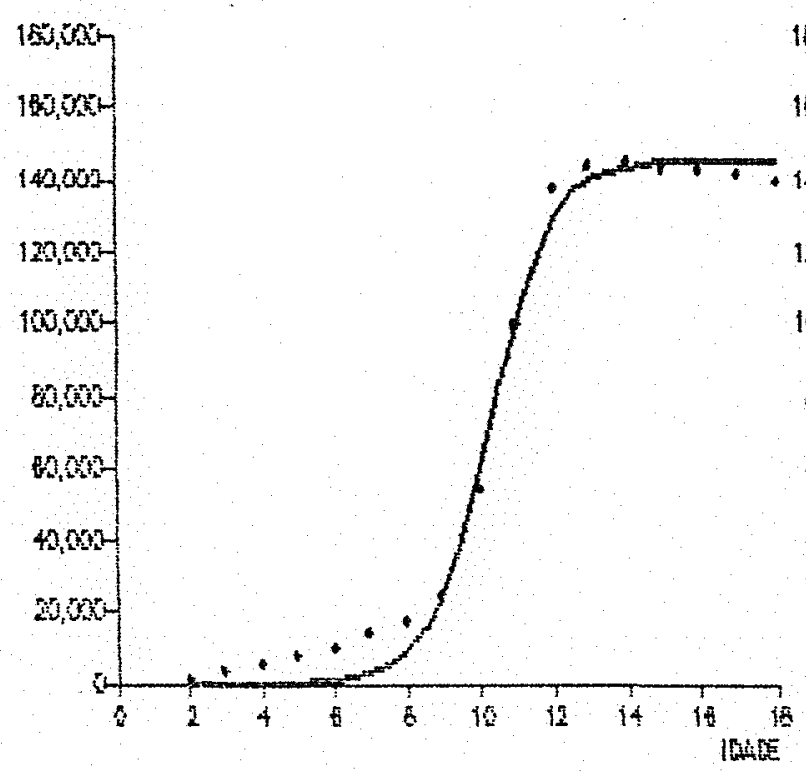

EXPERIMENTO 7

propution

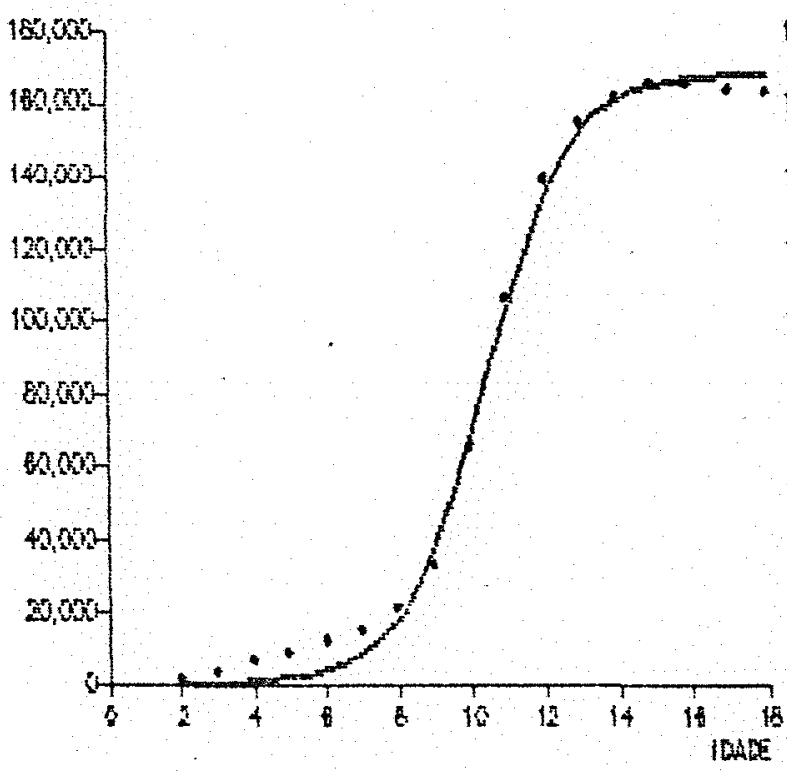

EXPERIMENTO 6

Proxitin

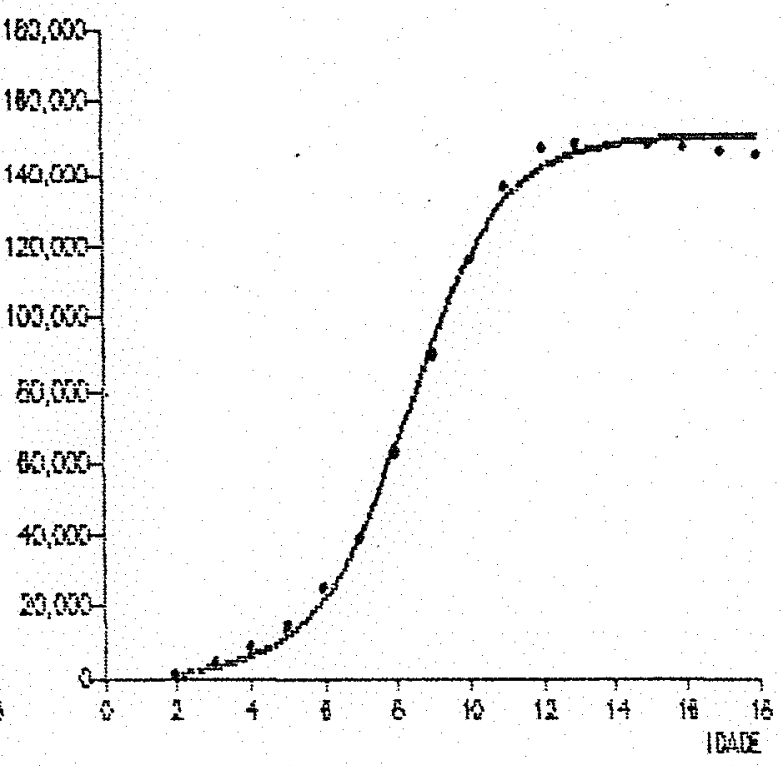

EXPERINENTO 8

Fonotin

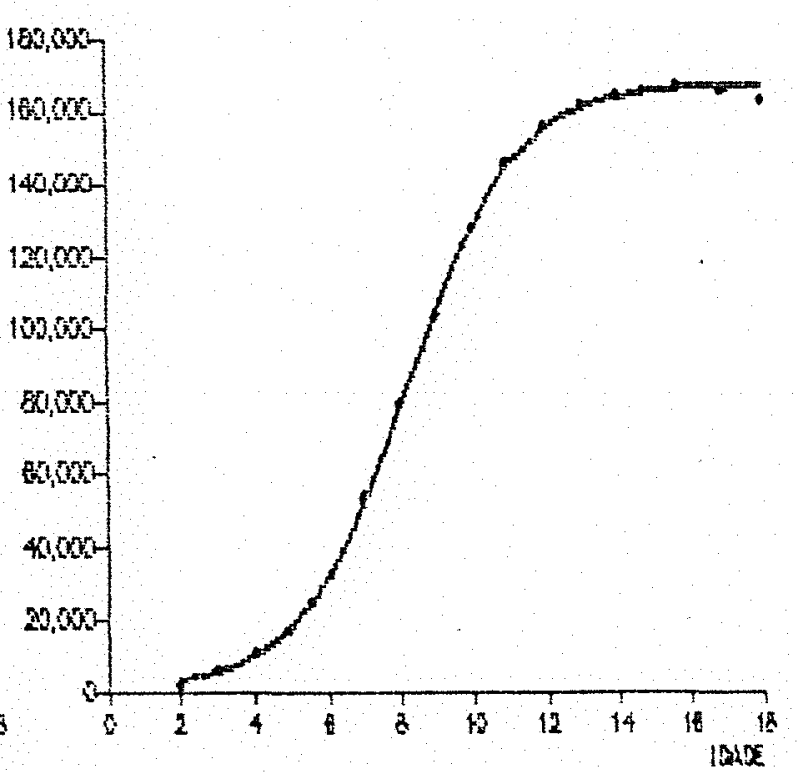

Fjogura 3 - Graficos das curvas estimaclas atraves da funsăo logrstica (a) scobrepostos aos pontos observados nos Experimentos $5,6,7,8$. 
Pronicio

EXPERIMENTO 1

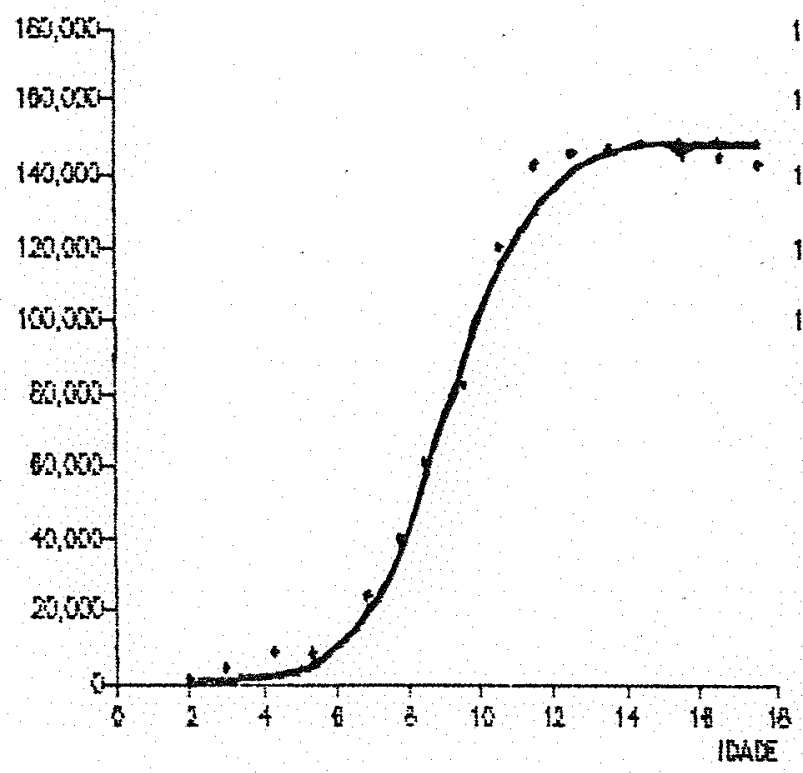

Provin

EXPERIMENTO 9

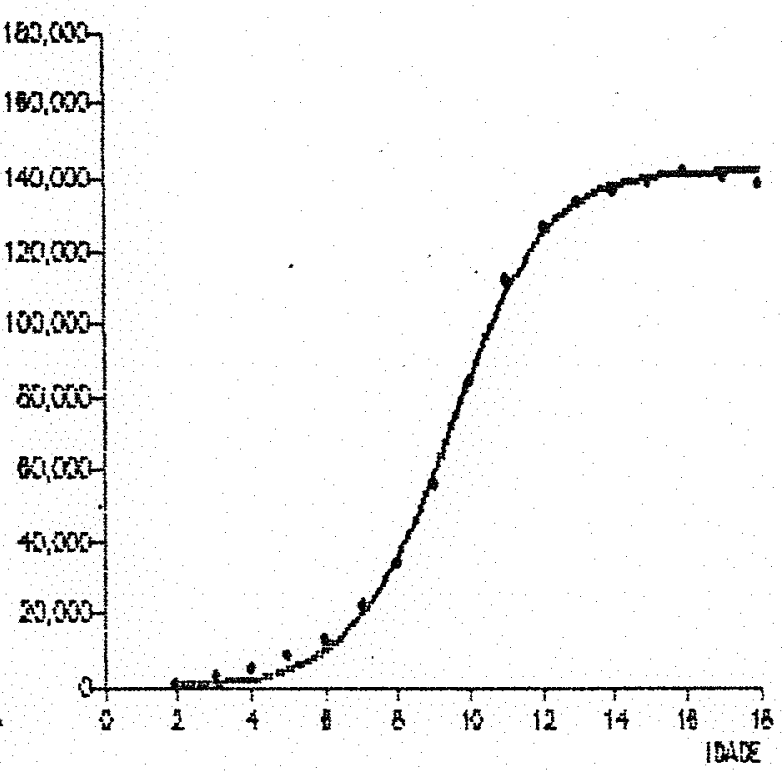

EXPERIMENTO 4

Phowin

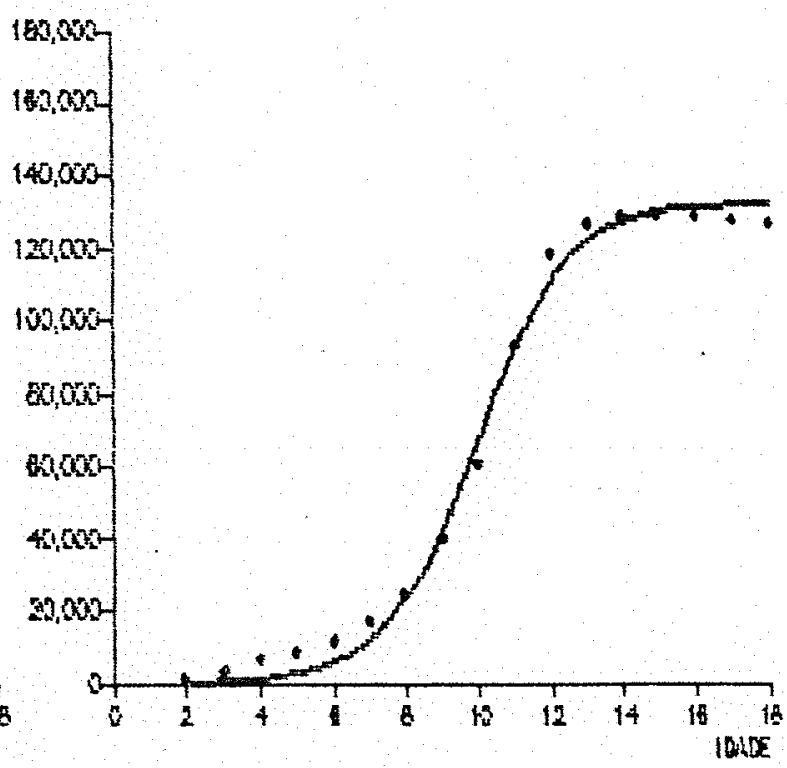

Figura 4 - Graficos das curvas estimadas atraves da funcáo logs stica (b) sobrepostos aos pontos cibservados nos Fxperimentos $1,9,3,4$. 


\section{EXPERIMENTO 5}

Foxulato

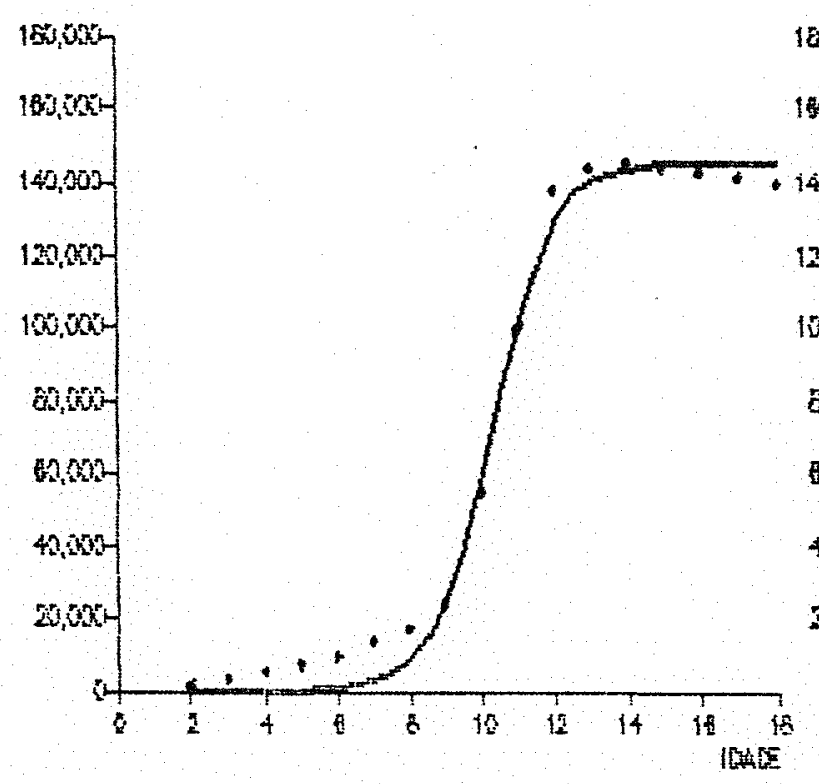

EXPERIMENTO 7

Pondentil

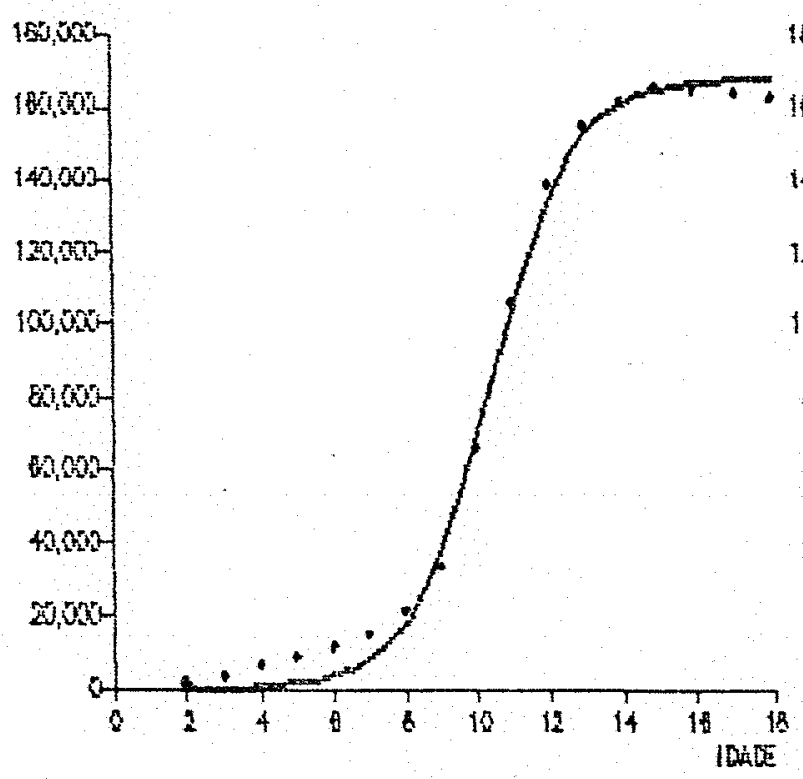

EXPERIMENTO 6

frourin

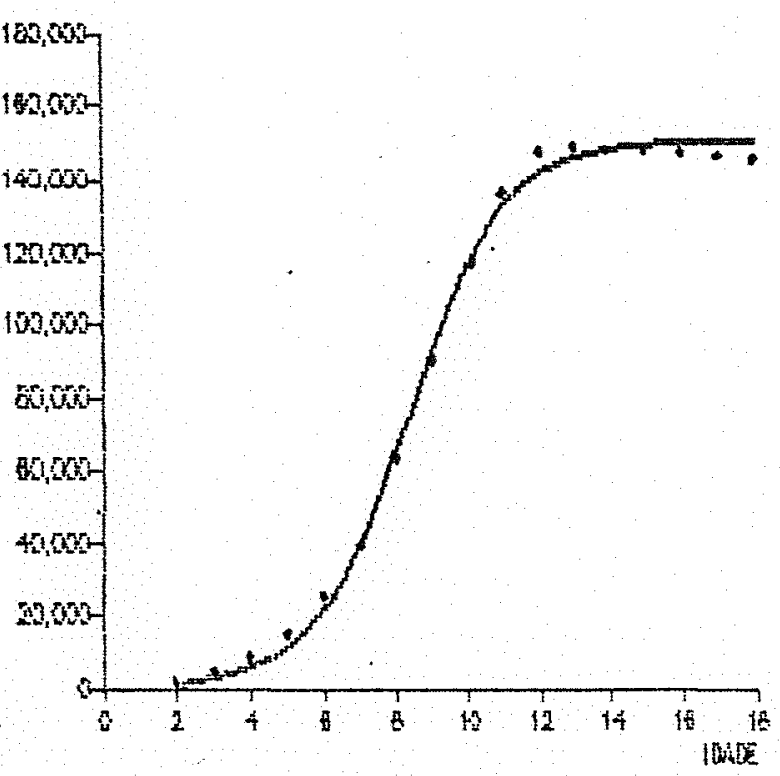

EXPERIMENTO 8

pranding

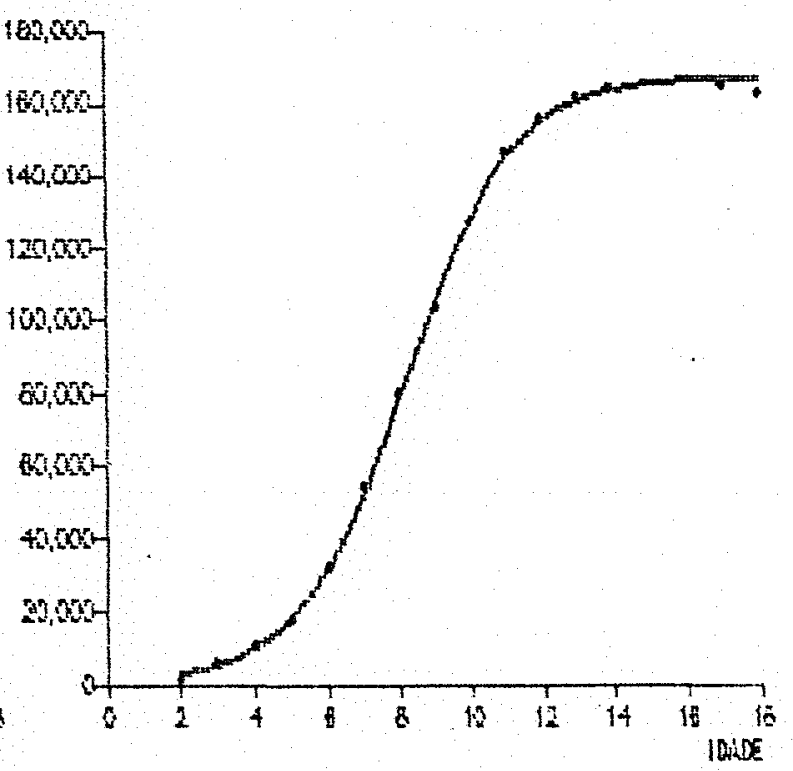

Figura g - oraficos das curvas estimadas atraves da runsăo logistica (b) sobrepostos aos pontos: observados nos fixperi meritos $5,6,7,8$. 
No Quadro 25 , estão os resultados da função logistica, na forma $\mathrm{Cl}$.

QUADRo 25 - Funçăo Logistica co) y $=\beta_{0} / C_{1}+e^{-C_{1}+\beta_{2} \times 3}$

\begin{tabular}{|c|c|c|c|c|c|c|}
\hline Exp. & $\begin{array}{c}\text { Estimati } \\
\hat{\beta}_{a}\end{array}$ & $\begin{array}{c}\text { vas dos } \\
\hat{\beta}_{1}\end{array}$ & $\begin{array}{r}\text { parametros } \\
\hat{\beta}_{2}\end{array}$ & QMR & $R^{2}$ & $\mathrm{u}$ \\
\hline 3 & 135165 & -7.036 & 0,636 & $8,806 \times 10^{7}$ & 0,9747 & 3 \\
\hline 4 & 131835 & $-7,662$ & 0.800 & $1,455 \times 10^{\circ}$ & 0,8584 & 38 \\
\hline 5 & 150117 & $-7,329$ & 0.716 & $1,121 \times 10^{\circ}$ & 0,9763 & 4 \\
\hline 6 & 150933 & $-7,603$ & 0,845 & $1,142 \times 10^{\circ}$ & 0,9738 & 16 \\
\hline 7 & 170730 & $-6,860$ & 0.673 & $4,701 \times 10^{7}$ & 0.9920 & 3 \\
\hline 8 & 163844 & $-8,206$ & 0,900 & $3,145 \times 10^{\circ}$ & 0.9385 & 14 \\
\hline 9 & 143729 & $-7,927$ & 0.792 & $9,533 \times 10^{7}$ & 0,9768 & 25 \\
\hline
\end{tabular}

Como foram utilizadas as mesmas estimativas dos parametros ajustados aos dados do Experimento 1 , como valores inlcials para 0 ajuste aos dados de todos os outros experimentos, isto ocasionou um bom ajuste da funça $\mathrm{Co}$ em al gurs exper imentos, enquanto que em outros. não fol täo bom. Deu-se a 1 dela de que a funçăo năo se ajusta multo bem as diversas formas de erescl merto. o que náo e verdade. 0 ajuste inefielente da fungao les a determinados experimentos, deve-se a imprecisáo dos valores inicials. Portanto, deve-se tormar cuidado na escolna dos valores inicials ao ajustar a funça los a dados de crescimento.

Sabemos que, se os valores inicials do parametro $\beta_{1}$ fossem escolnidos adequadamente em cada experimento $C_{1 c}=-1 n C \hat{\beta}_{1 a}$, as estimativas seriam multo proximas as das fumços anteriores Como os valores inicias adotados para ajustar a furça a todos os 
experimentos foram as estimativas encontradas no ajuste aos dados do Experimento 1 , as estimativas encontradas aqui, seguramente não são as do ponto de minimo absoluto. Da1, a grande desvantagem desta funçăo, que exıge do usuario, um certo conhecimento dos valores iniciais, a serem atribuidos aos parametros, para se conseguirem boas estimativas.

As Figuras 6 e 7 mostram os graficos dos ajustes da função na forma cel. Pode-se observar que o grafico do Experimento 9 , mostra uma curva estimada pela funçao, passando abaixo de 12 dos 17 pontos observados, certamente não e o mel hor ajuste da funça a esse corjunto de dados. A mesma situaça pode ser observada na figura 7 no graf 100 do Experimento 6 . Ja no Experimento \&, todos os portos observados eståo acima da curva estimada pela função. Isso evidencia que a escolna dos valores inicials para estimar os parametros desta funcão e de suma importancia. 


\section{Prowerial}

EXPERIMENTO 1

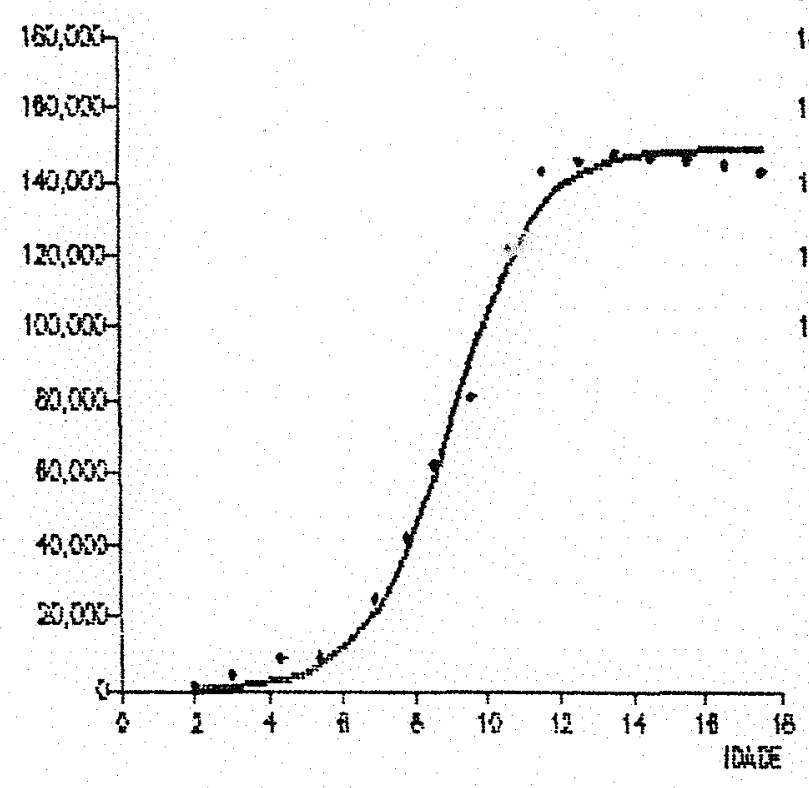

EXPERIMENTO 3

Foutir:

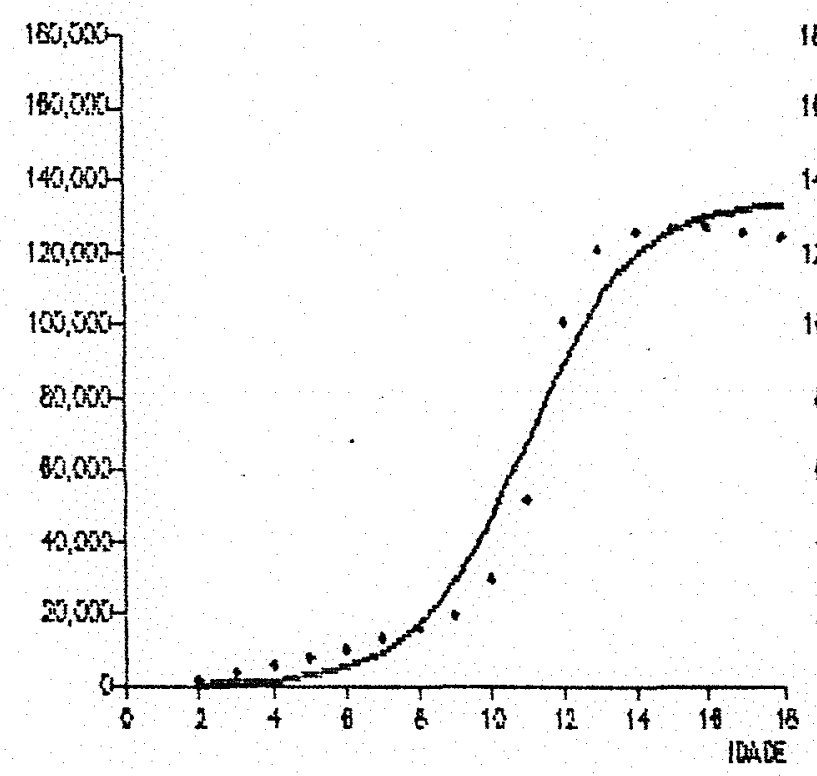

\section{Prontion}

EXPERIMENTO 9

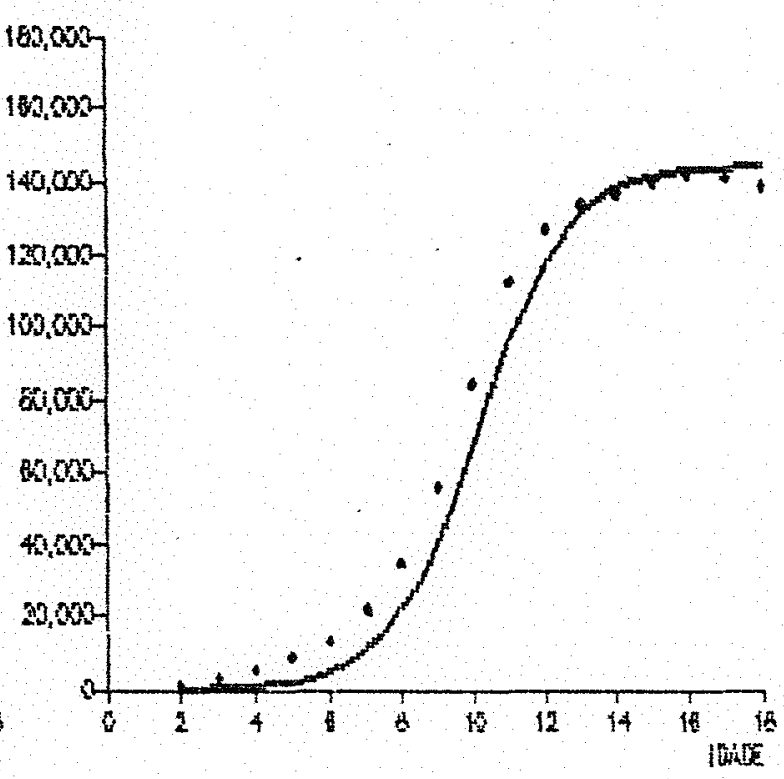

Pouting

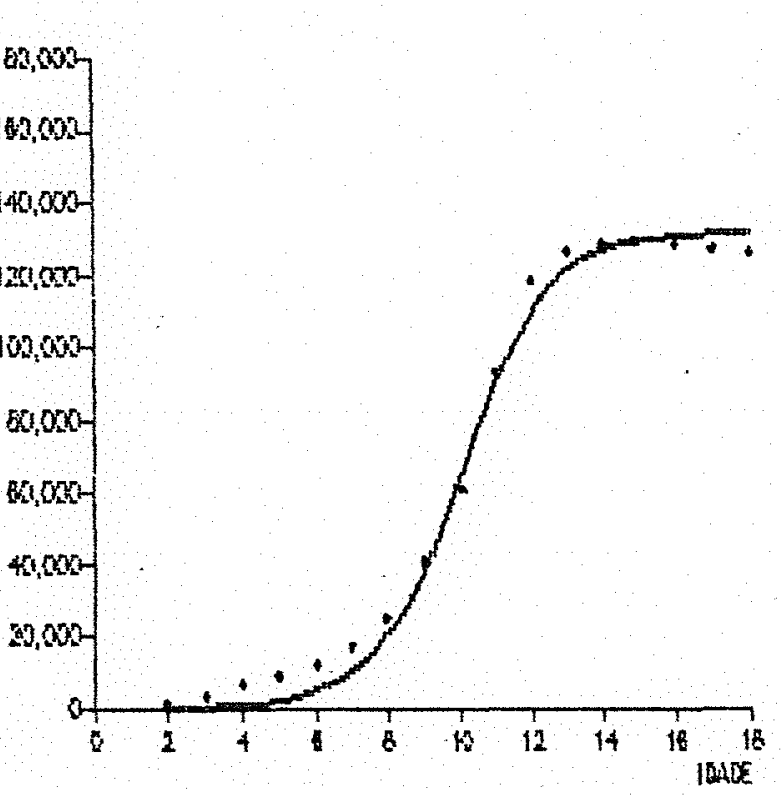

Figura 6 - Grásicos das curvas estimadas atraves da funcäl logistica (c) sobrepostos aos pontos observados nos Exporimentos $1,8,3,4$. 


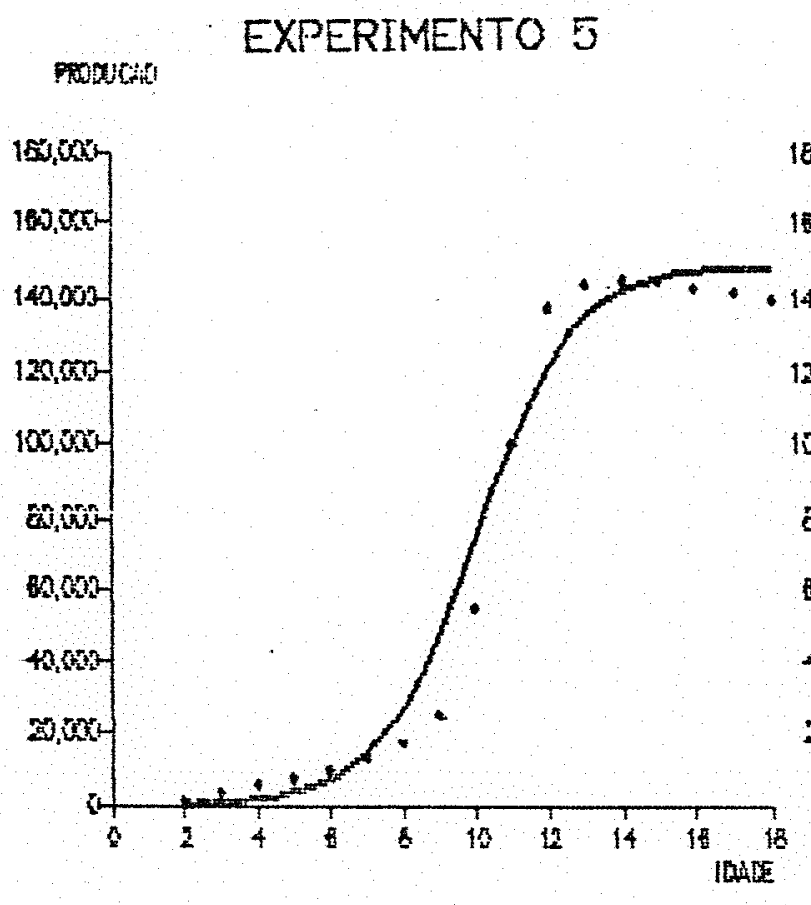

\section{EXPERIMENTO 7}

Patsitir

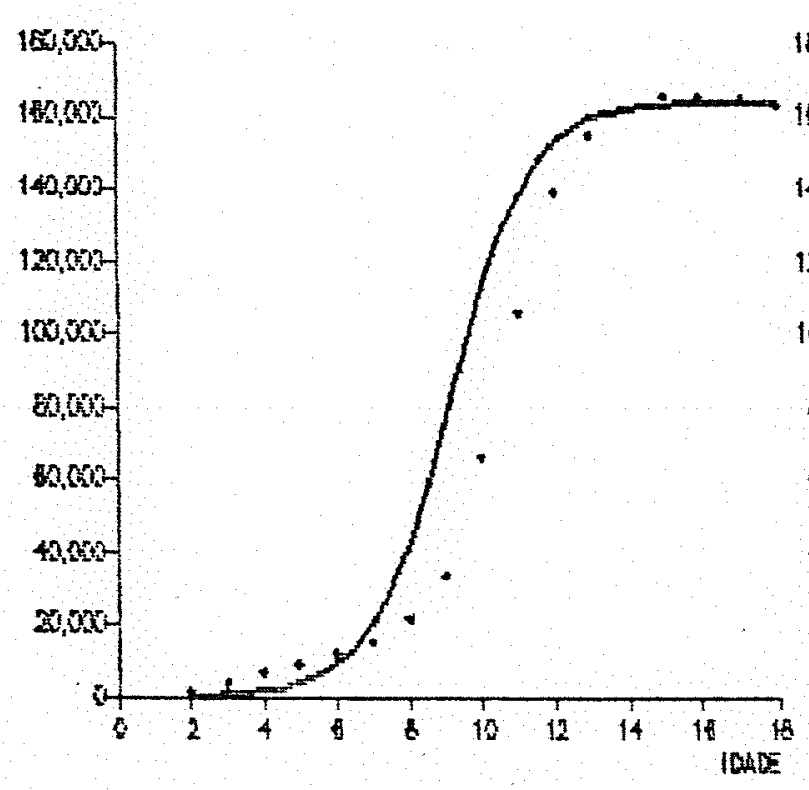

Fanding

EXPERIMENTO 6

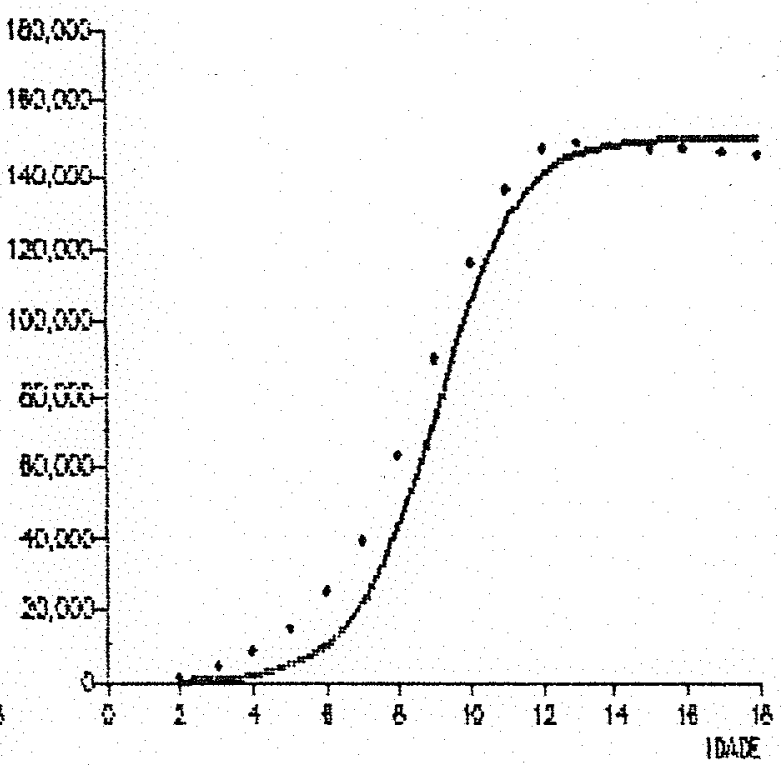

EXPERIMENTO 8

Proxtin

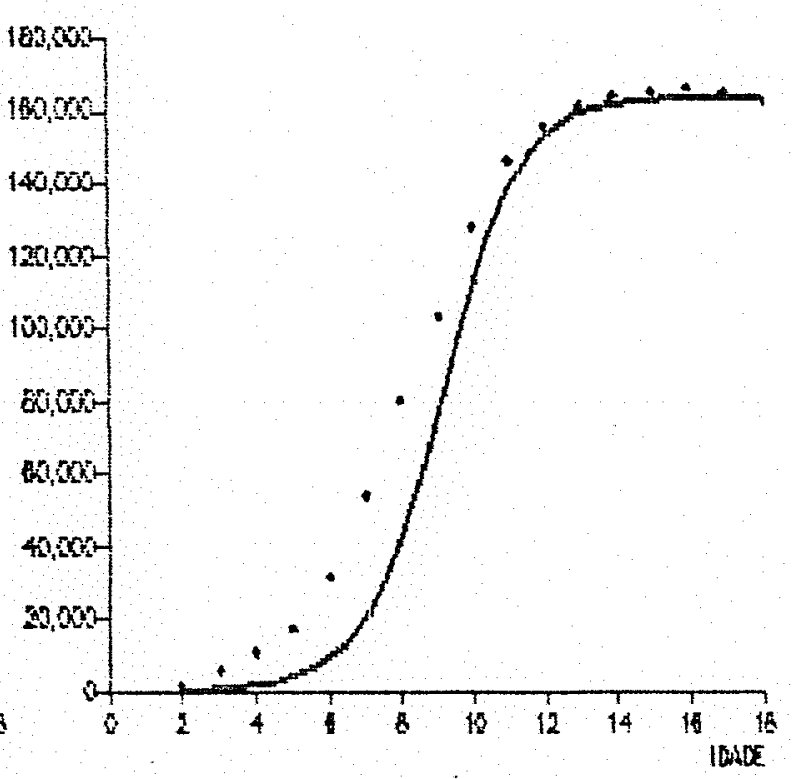

Figura 7 - Graficos das curvas estimadas atraves da runço logistica CCS sibrepostos aos poritos observados nos Fxperimentios 5,6,7,8. 
46.

Os resultados dos ajustes da função de Gompertz na forma $(d)$, aos dados dos experimentos estão no Quadro 26 .

QUADRO 26 - Função de Gompertz $C d$ y $=e^{C \beta_{0}}-\beta_{1} \beta_{2} S$

\begin{tabular}{lccccccc}
\hline Exp. & Estimativas dos parametros & QMR & $R^{2}$ & u \\
& $\beta_{0}$ & $\beta_{1}$ & $\beta_{2}$ & & & \\
\hline 3 & 11,780 & 2795 & 0,469 & $8,617 \times 10^{7}$ & 0,9753 & 33 \\
4 & 11,810 & 133 & 0,585 & $6,027 \times 10^{7}$ & 0,9828 & 15 \\
5 & 11,890 & 6615 & 0,407 & $7,466 \times 10^{7}$ & 0,9842 & 46 \\
6 & 11,940 & 42 & 0,606 & $4,594 \times 10^{7}$ & 0,9895 & 10 \\
7 & 12,050 & 356 & 0,545 & $0,181 \times 10^{7}$ & 0,9895 & 13 \\
8 & 12,050 & 23 & 0,649 & $2,031 \times 10^{7}$ & 0,9960 & 15 \\
9 & 11,890 & 70 & 0,610 & $3,162 \times 10^{7}$ & 0,9922 & 16 \\
\hline
\end{tabular}

Embora a função de Gompertz seja mals apropriada para descrever curvas de erescimento animal, ela ajustou-se bem em todos os experimentos sendo ligeiramente melhor nos experimentos cujo erescimento foi mais lento.

A assintota superior e dada por $e^{\hat{\beta}}$, cujos valores superam as produçóes maximas observadas em cada experimento. Por exemplo, no Experimento 3 , a produção maxima observada fol $128000 \mathrm{~kg}$, enquanto que a assintota superior fol $130600 \mathrm{~kg}$. O ponto de inflexäo, e dado pelas coordenadas: $x=-\ln \hat{\beta}_{1} / n \hat{\beta}_{2}, y=e^{\left(\beta_{0}-1\right)}$.

A curva dessa fumção e assimetrica em relação ao ponto de inflexão. Aqui, o ponto de inflexão acontece antes quando comparado aos das funços 
47.

simétricas. As Figuras 8 e 9 mostram os gráficos dos ajustes da função compertz na forma ldo aos dados dos experimentos. 


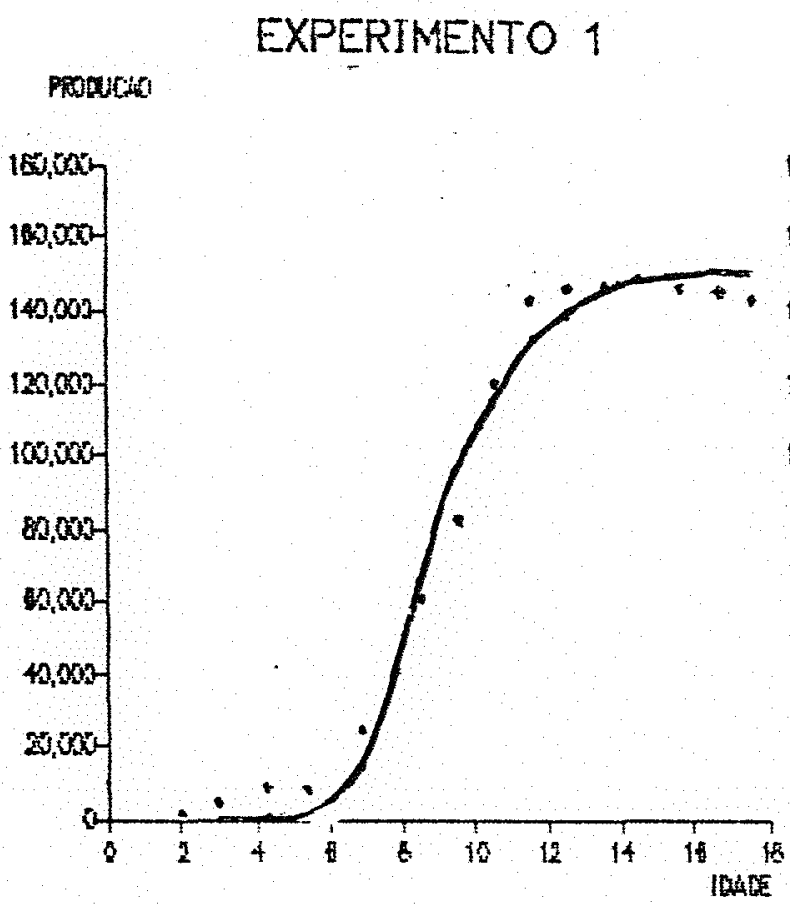

PRoracia

EXPERIMENTO 9

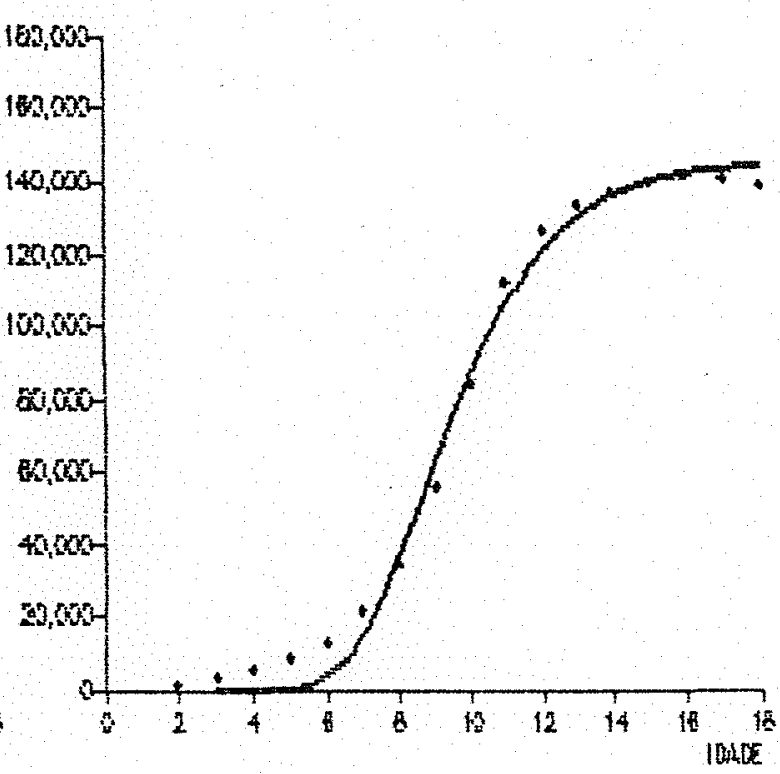

EXPERIMENTO 3

Fofterin

ponotin
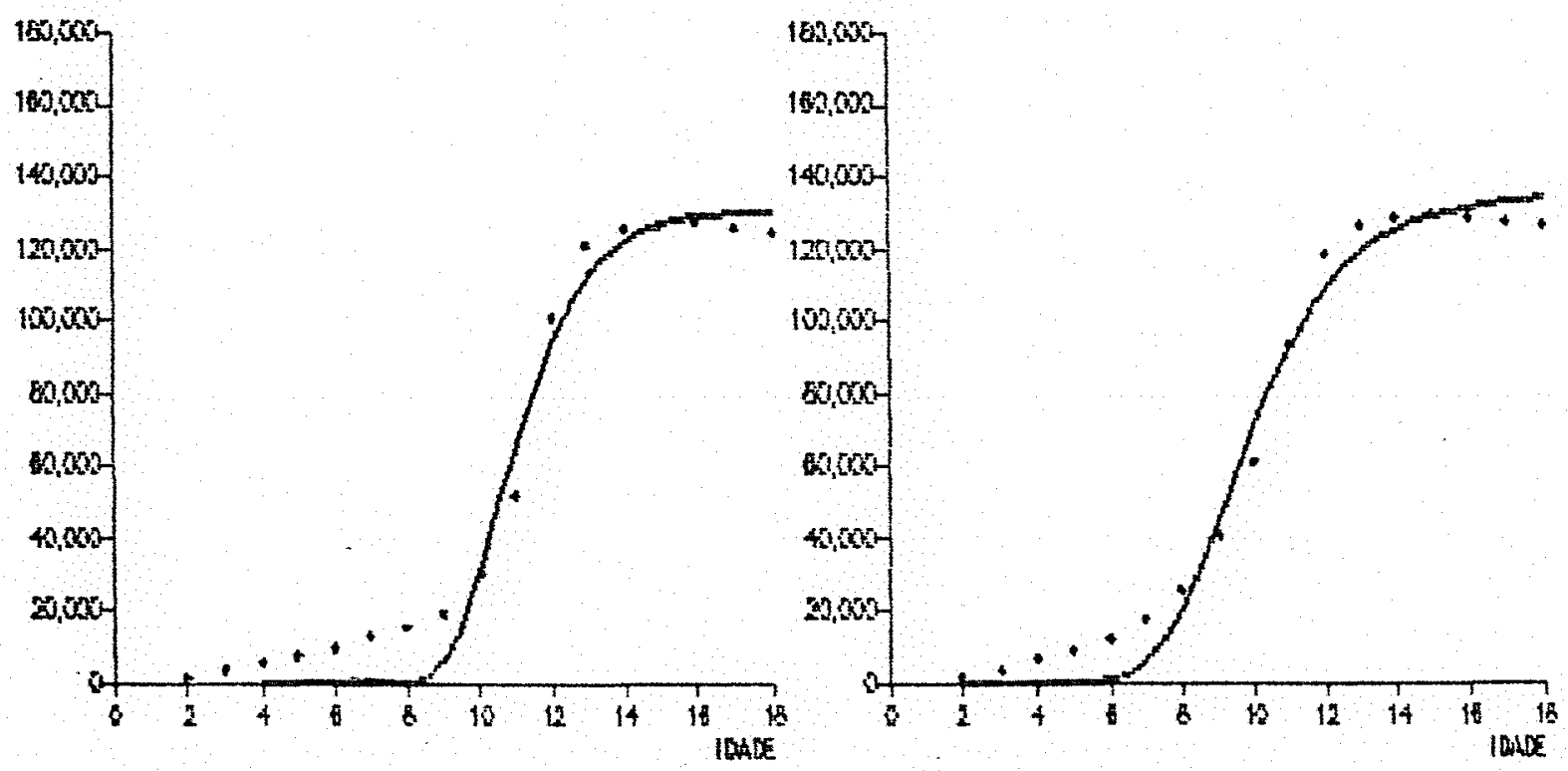

Fj.gura 8 -- Grafiros das curvas estimadas atraves da funçio Gompertz (d) sobrepostos fos pontos observados nos Fxperi mentess $1,9,3,4$. 
EXPERIMENTO 5

Proxtix

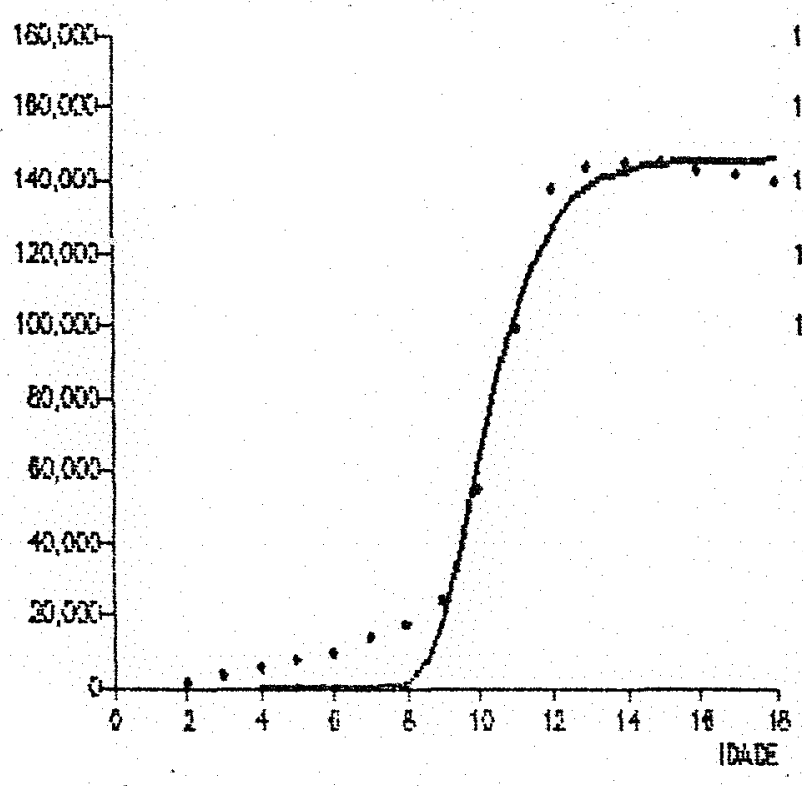

EXPERIMENTO 7

Panterit

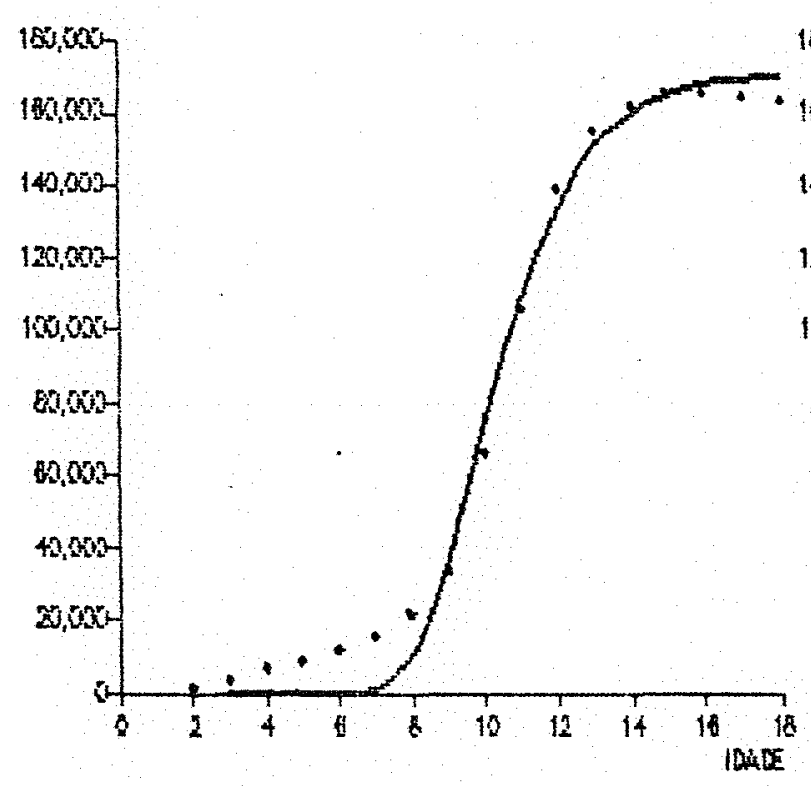

EXPERIMENTO 6

Fmoutin

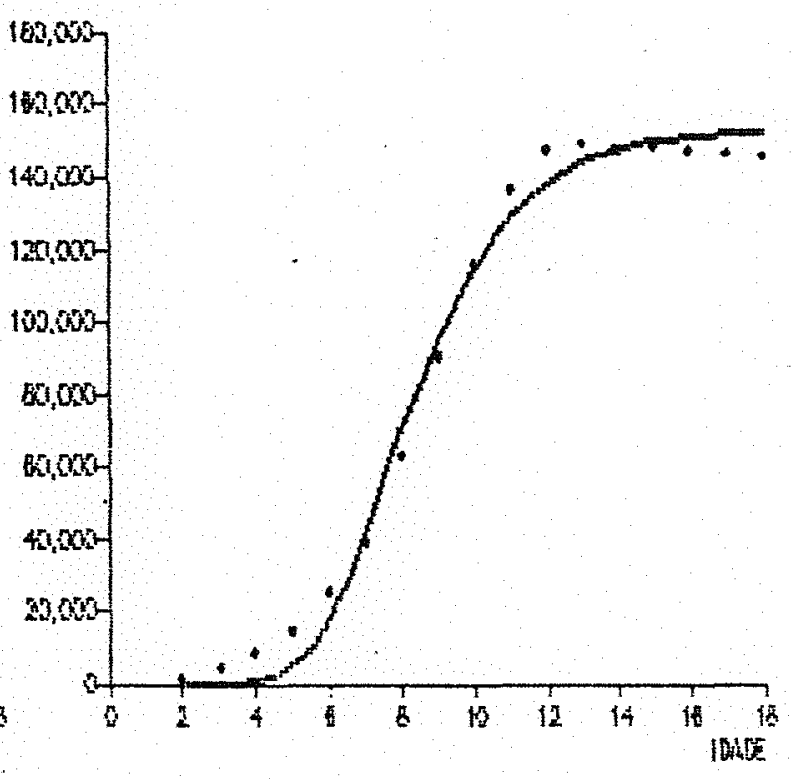

EXPERIMENTO 8

Panden

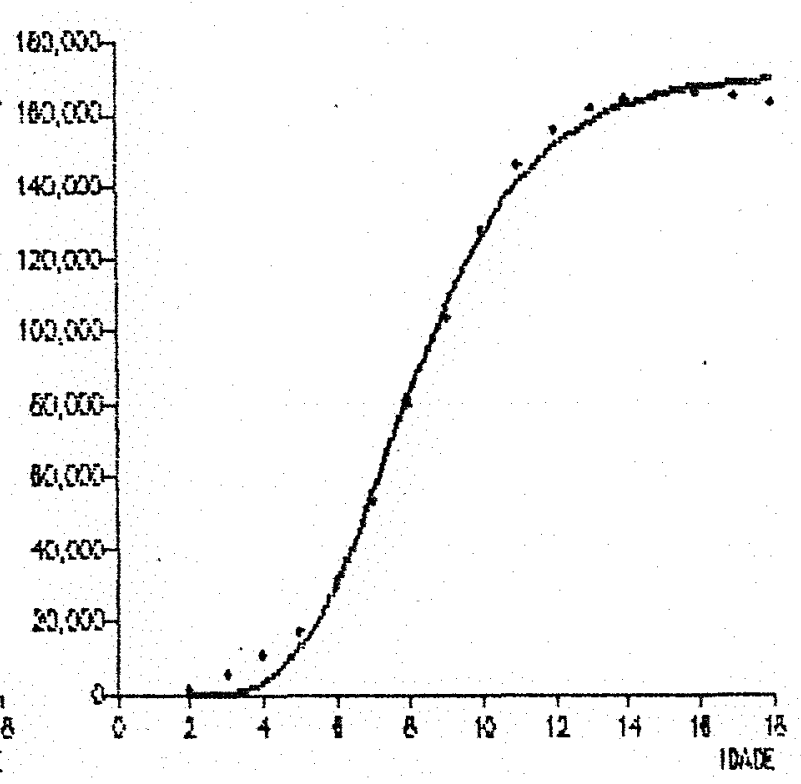

Figura $\theta$ - Gásicos das curvas estimadas atraves da funçäo Gompertz (d) sobrepostos aos pontos obsier vados nos Fixperimentos $5,6,7,8$. 
50.

0 Quadro 27 mostra os resultados dos ajustes da funçăo Gompertz na forma $l e$ aos dados dos experimentos.

QUADRO 27 - Função de Gompertz $\operatorname{ces} y=\beta_{0} e^{-\beta_{1} e^{-\beta} z^{x}}$

\begin{tabular}{|c|c|c|c|c|c|c|}
\hline Exp & $\begin{array}{l}\text { Estimativas } \\
\hat{\beta}_{o}\end{array}$ & $\begin{array}{c}\mathrm{dos} \\
\beta_{1}\end{array}$ & $\begin{array}{c}\text { parametros } \\
\beta_{2}\end{array}$ & QMR & $\mathrm{R}^{2}$ & $\mathrm{U}$ \\
\hline 3 & 130790 & 2794 & 0,755 & $8,617 \times 10^{7}$ & 0,9753 & 32 \\
\hline 4 & 134627 & 133 & 0,535 & $6.027 \times 10^{7}$ & 0,9828 & 14 \\
\hline 5 & 146076 & 6615 & 0,897 & $7,466 \times 10^{7}$ & 0.9842 & 43 \\
\hline 6 & 153482 & 42 & 0,500 & $4,594 \times 10^{7}$ & 0,9895 & 10 \\
\hline 7 & $17075 e$ & 356 & 0,605 & $6,181 \times 10^{7}$ & 0,9895 & 20 \\
\hline 8 & 171745 & 23 & 0,431 & $2,031 \times 10^{7}$ & 0.9960 & 14 \\
\hline 9 & 145791 & 70 & 0,494 & $3,162 \times 10^{7}$ & 0,9922 & 10 \\
\hline
\end{tabular}

Apresenta as mesmas caracteristicas da funça na forma cds, com relaçáo ao ajuste. Praticamente os mesmos valores para $\beta_{1}$.

A assintota superior e dada por $\hat{\beta}_{0} e$ ponto de inflexão por $x=\ln \hat{\beta}_{1} \hat{\beta}_{2}, y=\hat{\beta}_{0}$, cujos valores aproximam aos da função na forma (d). Os graficos do ajuste da funça Gompertz na forma les estão nas figuras 10 e 11. 


\section{EXPERIMENTO 1}

PMontio

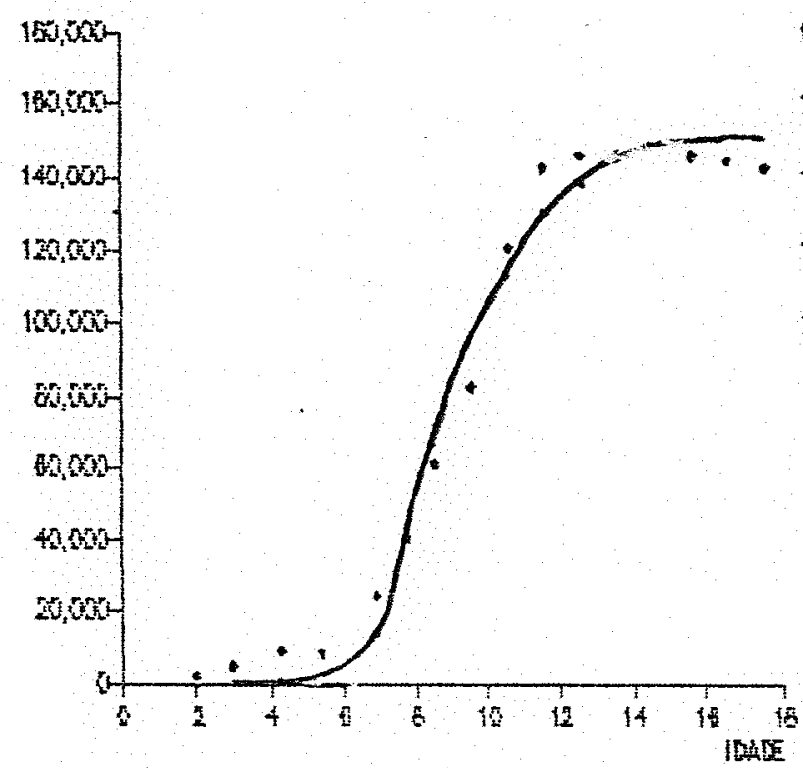

\section{EXPERIMENTO 3}

movetid

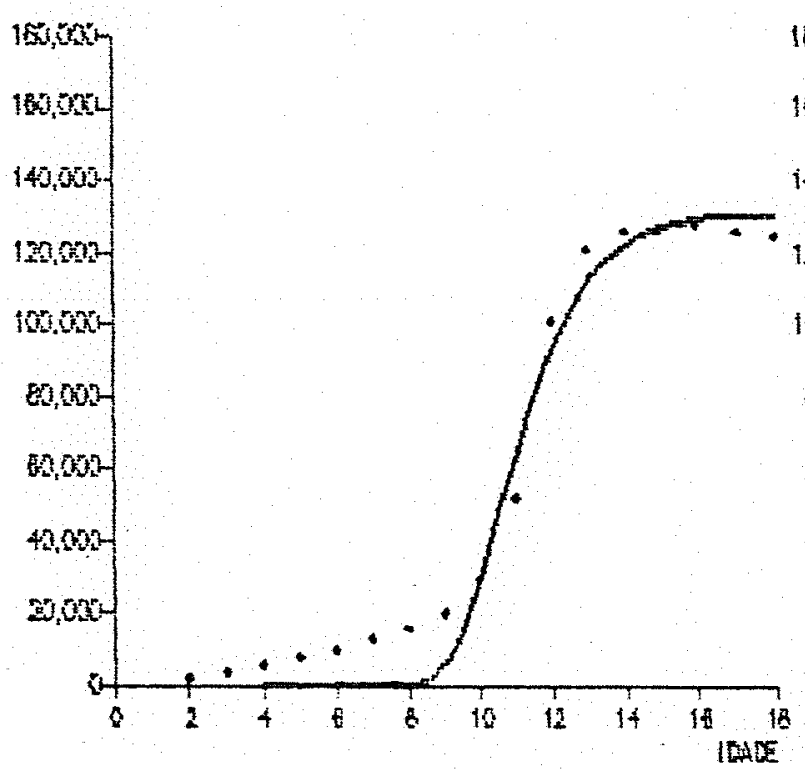

\section{EXPERIMENTO 9}

$\operatorname{Panx}$

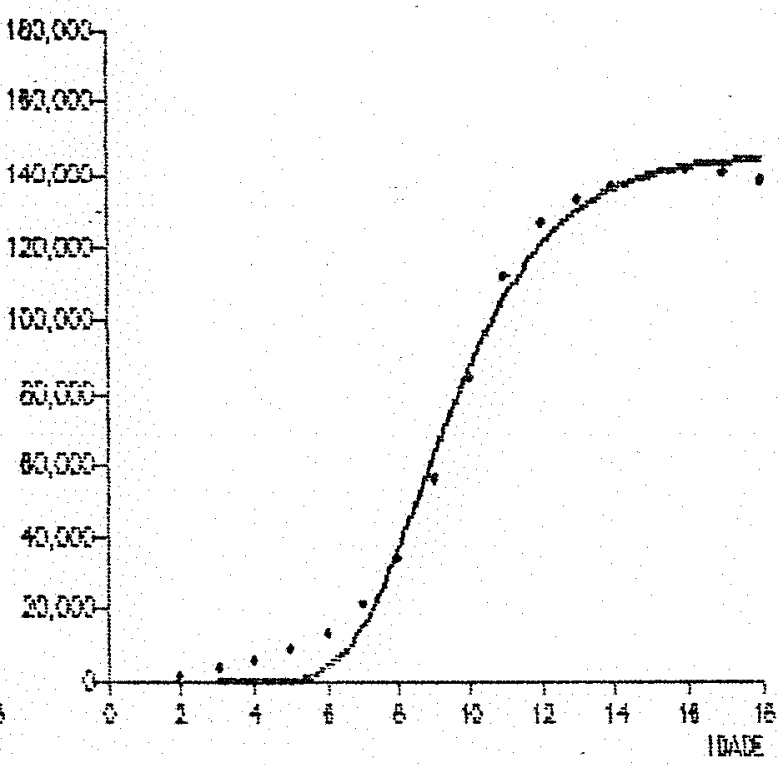

EXPERIMENTO 4

Proturing

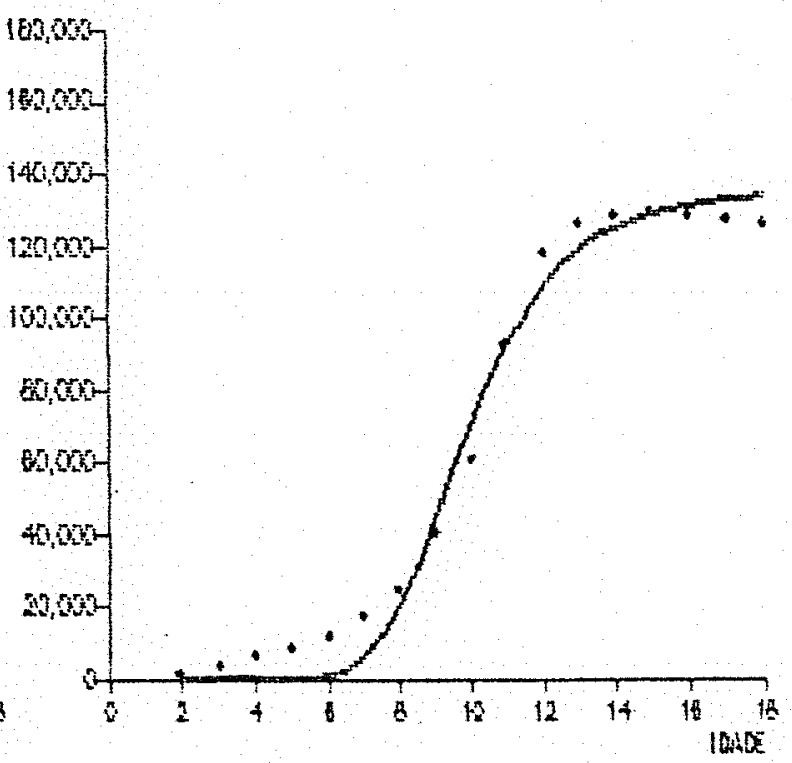

Figura 10 - oraricos das curvas estimadas atraves da runçác Eomperlo $(e)$ sobrepostos fos pontios observados nos Experimentos $1,9,3,1$. 


\section{EXPERIMENTO 5}

monutir

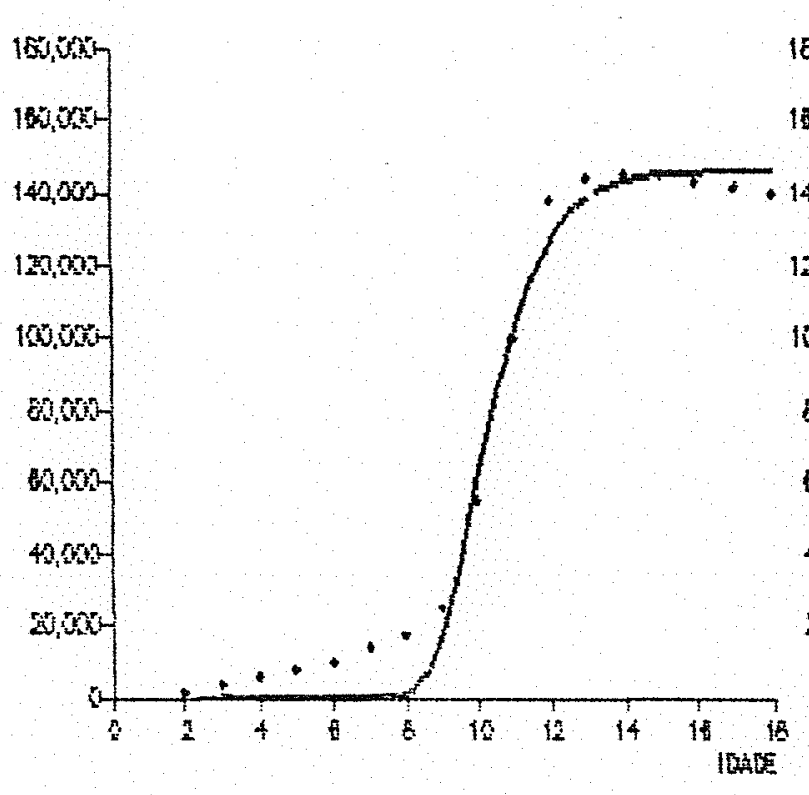

EXPERIMENTO 7

proted

\section{Fandin \\ EXPERIMENTO 6}

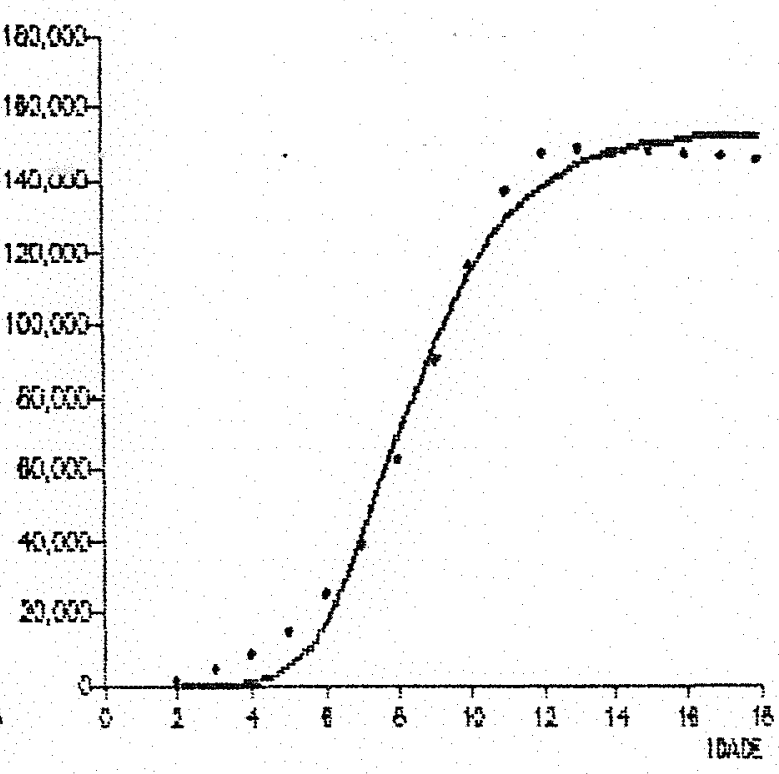

EXPERIMENTO 8

mantom
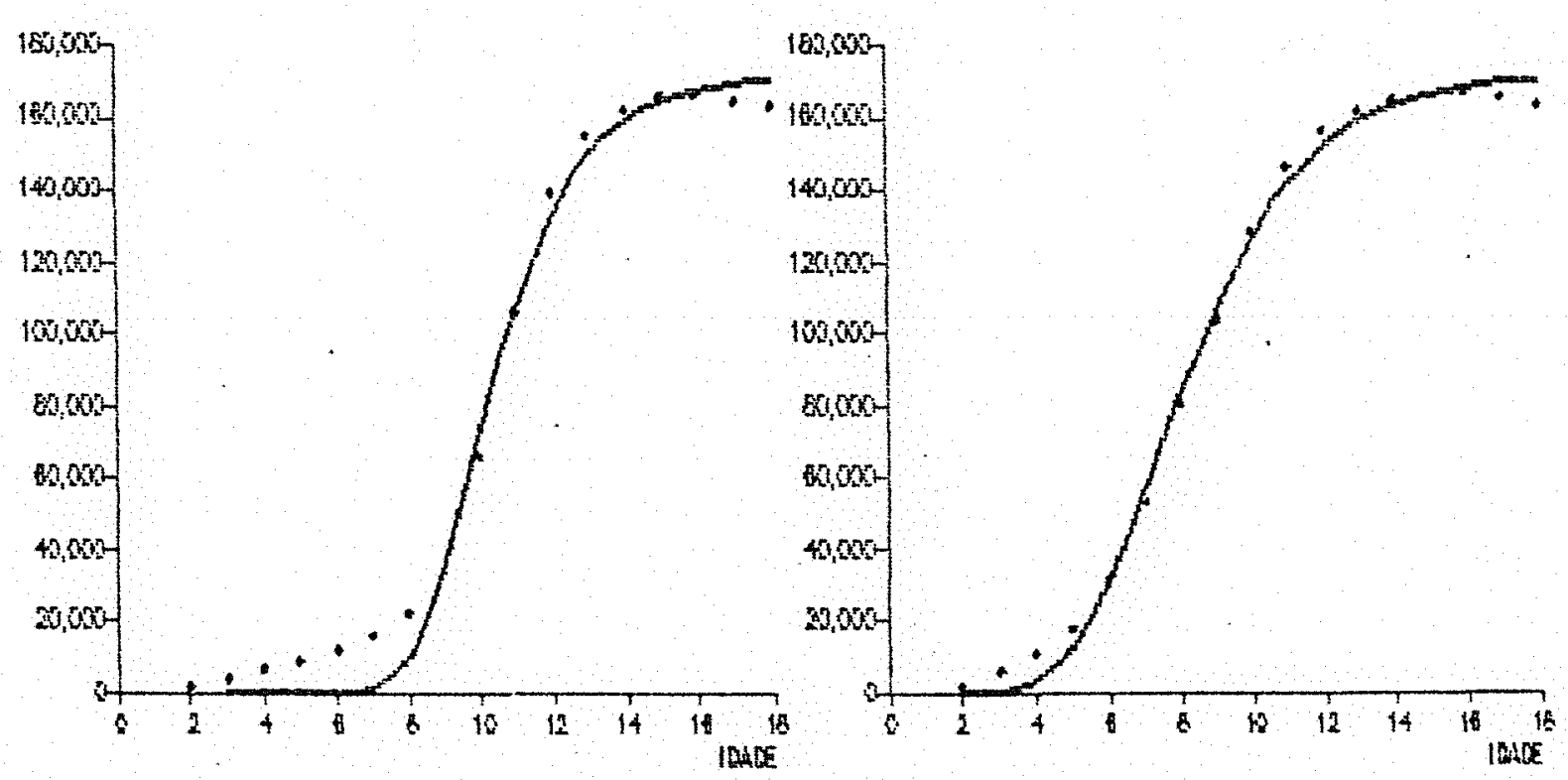

Fi.gura 11 - Graficos das curvas estimadas atraves da funça compertz $(e)$ sobrepositos aos pontos observados nos: Fixper i mentos $5,6,7,8$. 
53.

No Quadro 28 estão os resultados dos ajustes da funç̃o monomolecular aos dados dos experimentos.

QUADRo 28 - Função Monomolecular $(f) y=\beta_{0}\left(1-\beta_{1} e^{-\beta_{2}}\right)_{2}$

Exp. Estimativas dos parametros $\beta_{0} \quad$ Qink $\beta_{2} R^{2} \quad u$

\begin{tabular}{|c|c|c|c|c|c|}
\hline $7,450 \times 10^{\circ}$ & 1,000 & $1,367 \times 10^{-5}$ & $4.619 \times 10^{\circ}$ & 0.8674 & 1500 \\
\hline 1230531 & 1,032 & 0.009 & $3,503 \times 10^{\circ}$ & 0,8090 & 155 \\
\hline 55956536 & 1,001 & $2,100 \times 10^{-4}$ & $6,531 \times 10^{\circ}$ & 0.8617 & 14 \\
\hline 241300 & 1,277 & 0,079 & $3.598 \times 10^{\circ}$ & 0,9175 & \\
\hline $2,509 \times 10^{\circ}$ & 1,000 & $5,360 \times 10^{-5}$ & $5,997 \times 10^{6}$ & 0,8978 & 130 \\
\hline 203203 & 1.275 & 0,081 & $2.989 \times 10^{\circ}$ & 0,9415 & \\
\hline 524819 & 1.091 & 0,025 & $3,347 \times 10^{8}$ & 0,9174 & \\
\hline
\end{tabular}

A função monomolecular não apresenta ponto de inflexão e não se ajustou bem em quase todos os experimentos. O ajuste fol melhor nos experimentos cujo crescimento $f 01$ mais lento. CExperimentos 8 e 8 . Foram usadas tambem as estimativas obtidas no Experimento 8. como valores inlclals para ajustar aos dados dos demais experimentos, na tentativa de melhorar o ajuste, no entanto as estimativas encontradas aqui foram as mesmas do quadro 28 , ou seja, não houve melhora. A assintota superior dada pela estimativa de $\beta_{0} C_{0} \hat{\beta}_{0}$ e muito superior aos valores das producóes maximas observadas em cada experimerto. Nas flguras $12 \in 13$ estao os graficos referentes aos ajustes da funçäo monomolecular aos dados dos experimentos. 


\section{EXPERIMENTO 1}

ponouidi:

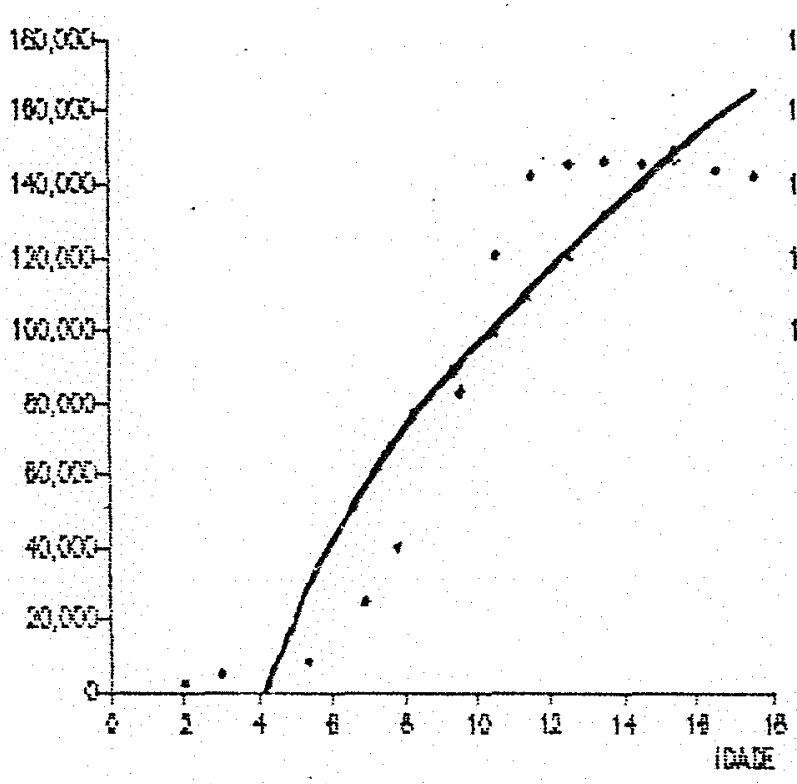

EXPERIMENTO 3

Pentir:

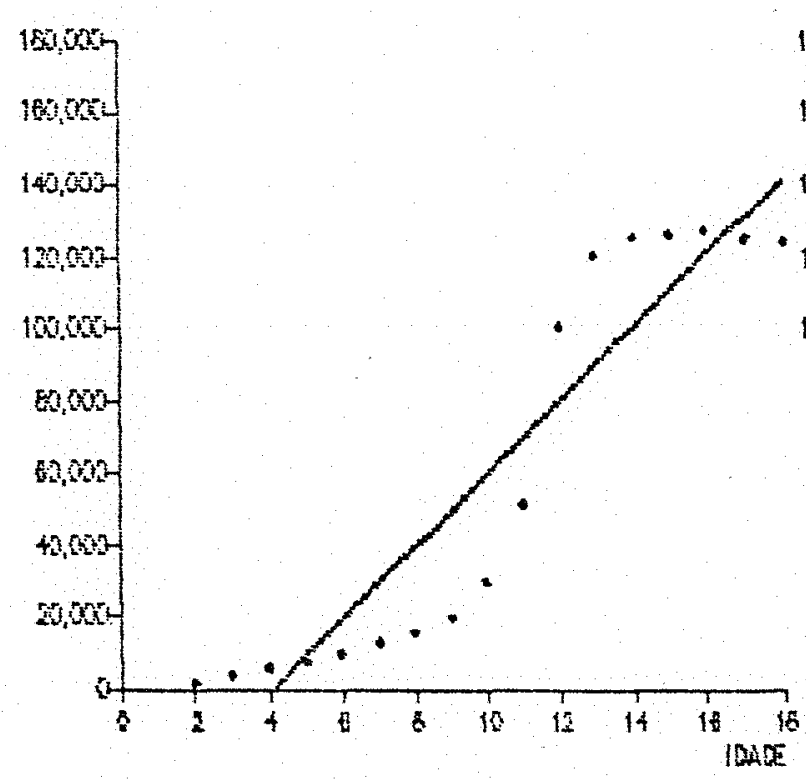

EXPERIMENTO 9

maxtin

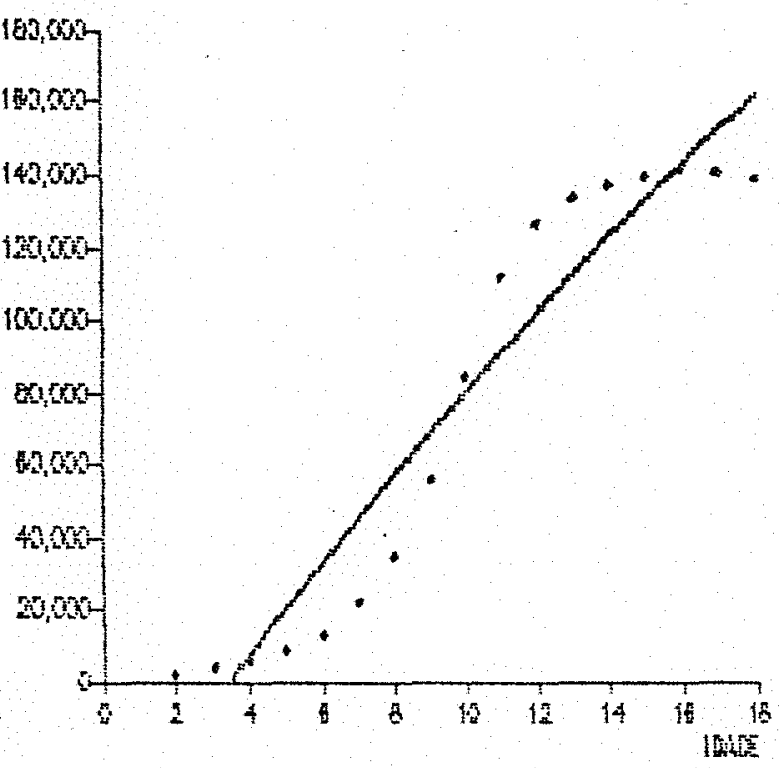

EXPERIMENTO 4

Fratain

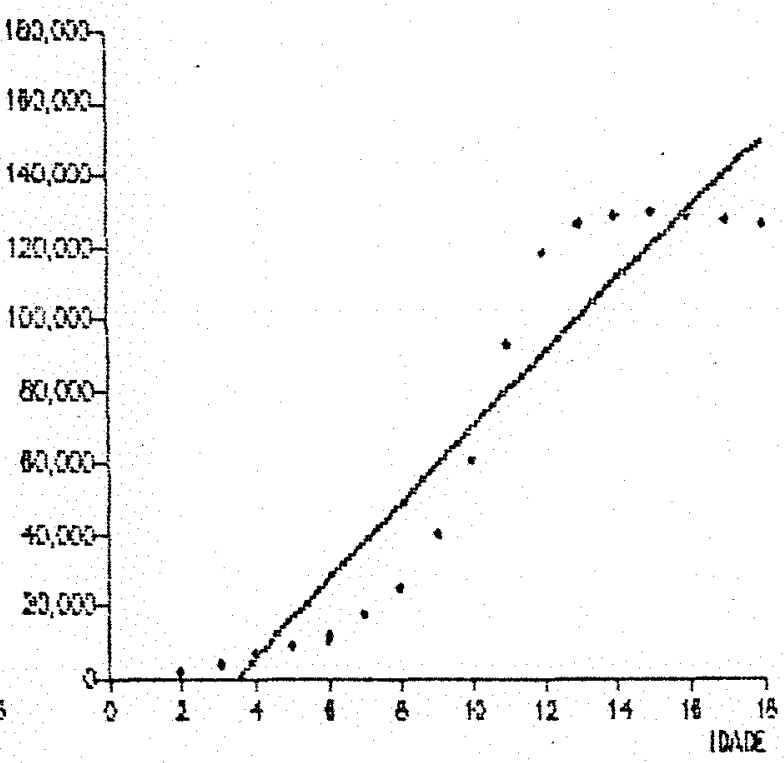

Figura 12 - Graficos das curvas estimadas atraves da funçáco monomol ecul ar sobrepostos aos pontos observados mosi Exporimentos 1, 9,3,4. 


\section{EXPERIMENTO 5}

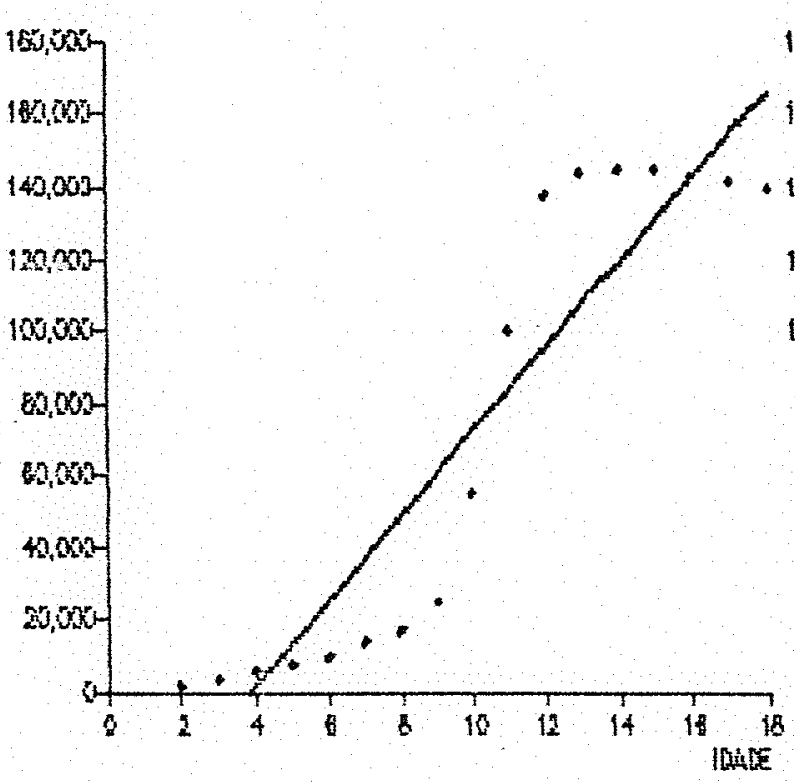

Prouch

EXPERIMENTO 6

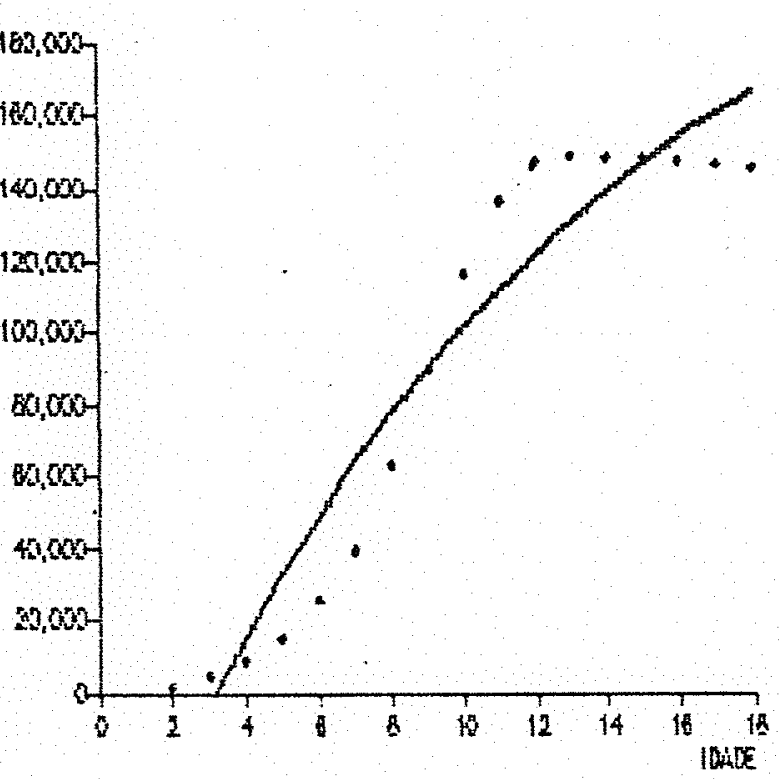

EXPERIMENTO 8

mantin

Prouch

EXPERIMENTO 7
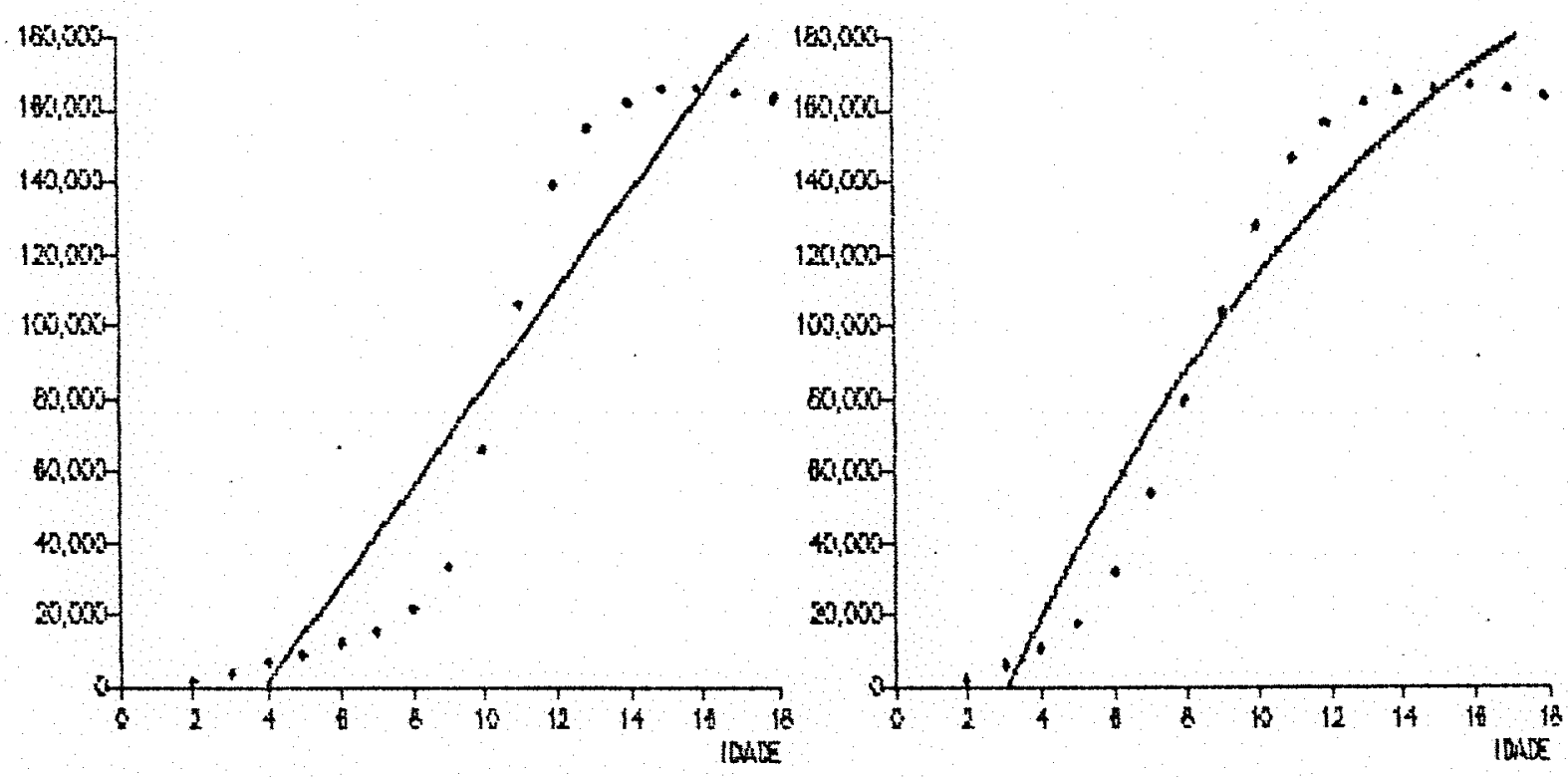

Fj.gura 13 - Graficos das curvas estimadas atraves da runşa monomolocular sobropostos aos portos observados nos Experimentos $5,6,7,8$. 
Os resultados do ajuste da função Brody estão no Quadro 29 .

\begin{tabular}{|c|c|c|c|c|c|c|}
\hline Exp. & $\begin{array}{l}\text { Estimati } \\
\text { sa }_{0}\end{array}$ & $\begin{array}{c}\text { vas dos } \\
\beta\end{array}$ & $\begin{array}{r}\text { parametros } \\
\hat{\beta}_{2}\end{array}$ & QMR & $R^{2}$ & $\mathrm{u}$ \\
\hline 3 & 4408979 & 4451885 & 0,002 & $4,658 \times 10^{\mathrm{B}}$ & 0,8663 & 603 \\
\hline 4 & 1230323 & 1269571 & 0.000 & $3,503 \times 10^{\circ}$ & 0,8999 & 161 \\
\hline 5 & 4408979 & 4455481 & 0,003 & $6.540 \times 10^{8}$ & 0,8615 & 603 \\
\hline 6 & 241297 & 308255 & 0,079 & $3,598 \times 10^{8}$ & 0,9175 & 25 \\
\hline 7 & 4409010 & 4462882 & 0,003 & $0.033 \times 10^{\circ}$ & 0.8972 & 852 \\
\hline 8 & 263200 & 335614 & 0,081 & $2.989 \times 10^{\mathrm{a}}$ & 0,9415 & 21 \\
\hline 9 & 524776 & 572343 & 0,025 & $3,347 \times 10^{8}$ & 0,9174 & 155 \\
\hline
\end{tabular}

As estimativas de $\beta_{0}$ na funcáo Brody, aproximam-se daquelas encontradas na funço monomolecular, nos experimentos cujo crescimento e mais iento e as estimativas são menores. Usario as estimativas do Experimento 8 como valores iniclals para 0 ajuste dos demais experimentos, obtem-se as nesmas estimativas do Quadro 20 os graficos da funcá Brody ajustada aos dados dos experimentos estao has figuras 14 e 15. 
EXPERIMENTO 1

Paxpucio

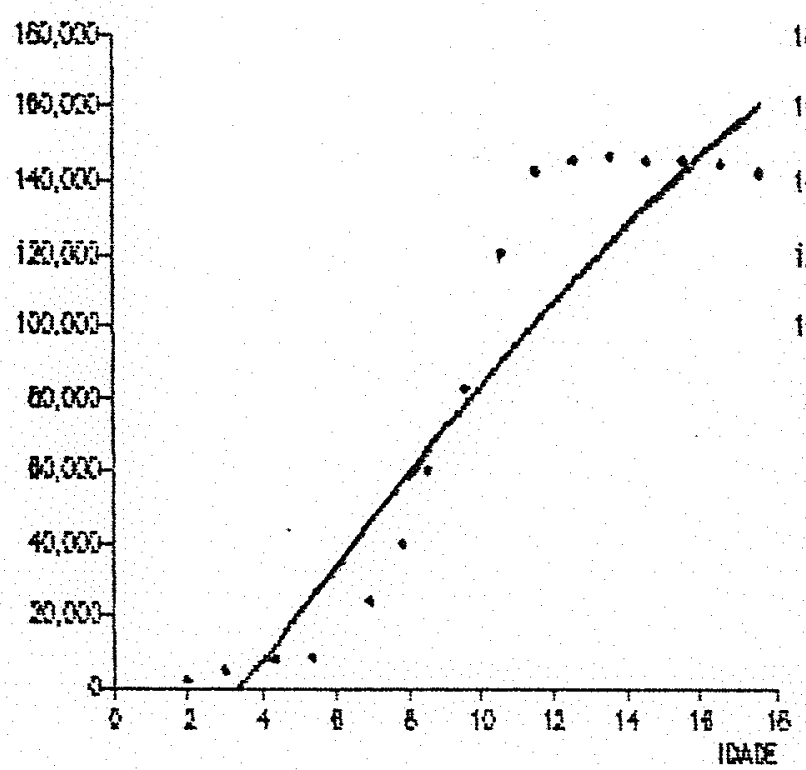

EXPERIMENTO 3

Phouch

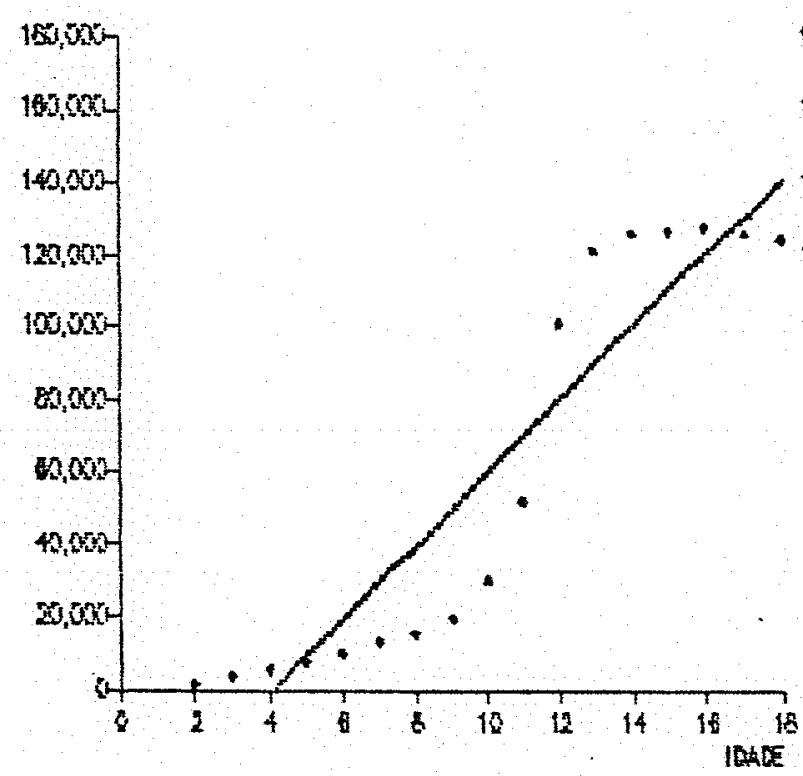

EXPERIMENTO 9

Frovent

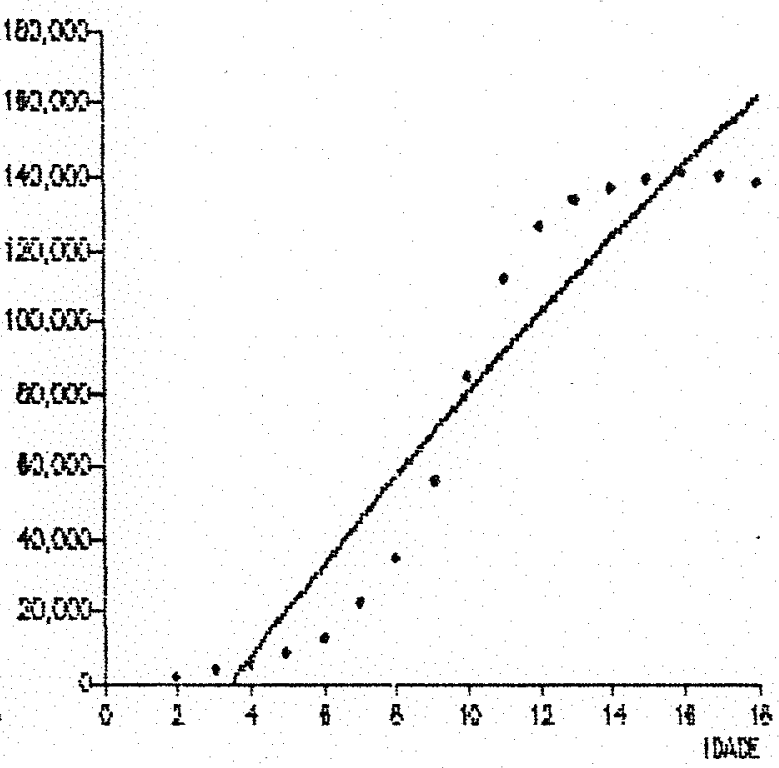

EXPERIMENTO 4

monerin

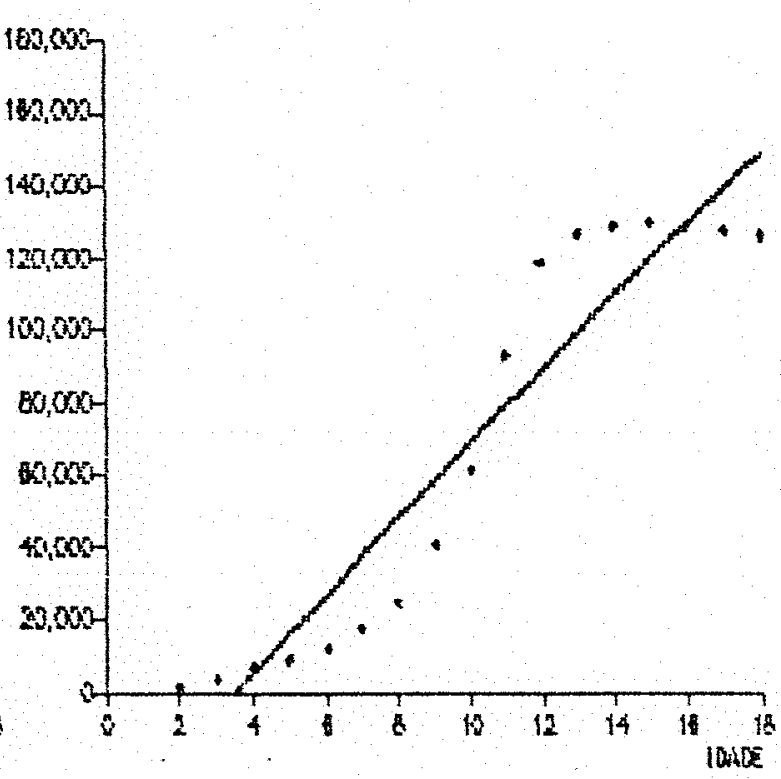

Figura 14 - Graficos das curvas estimadas atravess da funço Brody sobrepostos aos pontos observados nos fexperi mentoss $1,9,3,4$. 
EXPERIMENTO 5

Pandert
EXPERIMENTO 6

Fravian

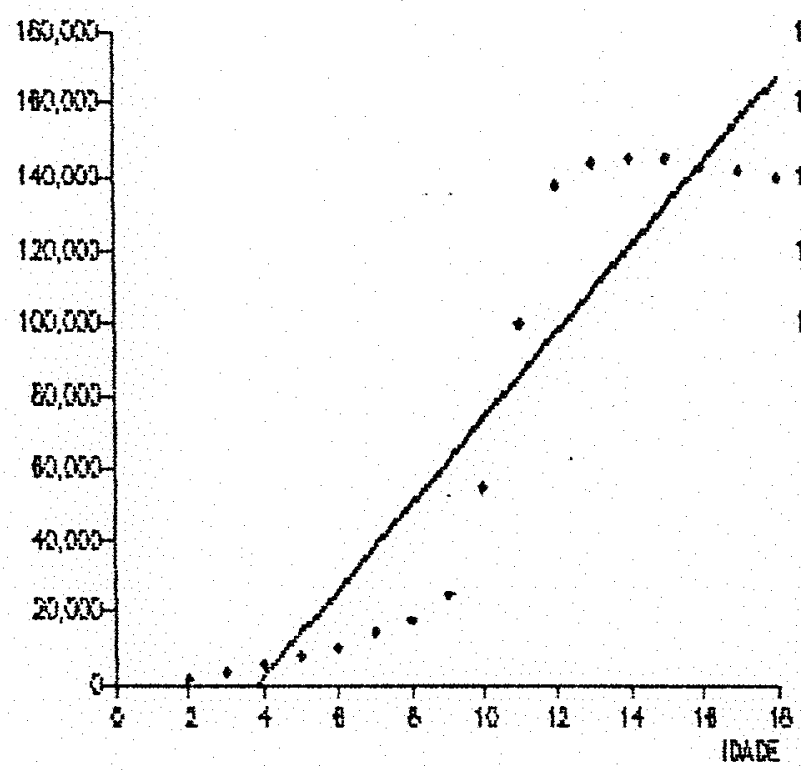

EXPERIMENTO 7

Parturit!

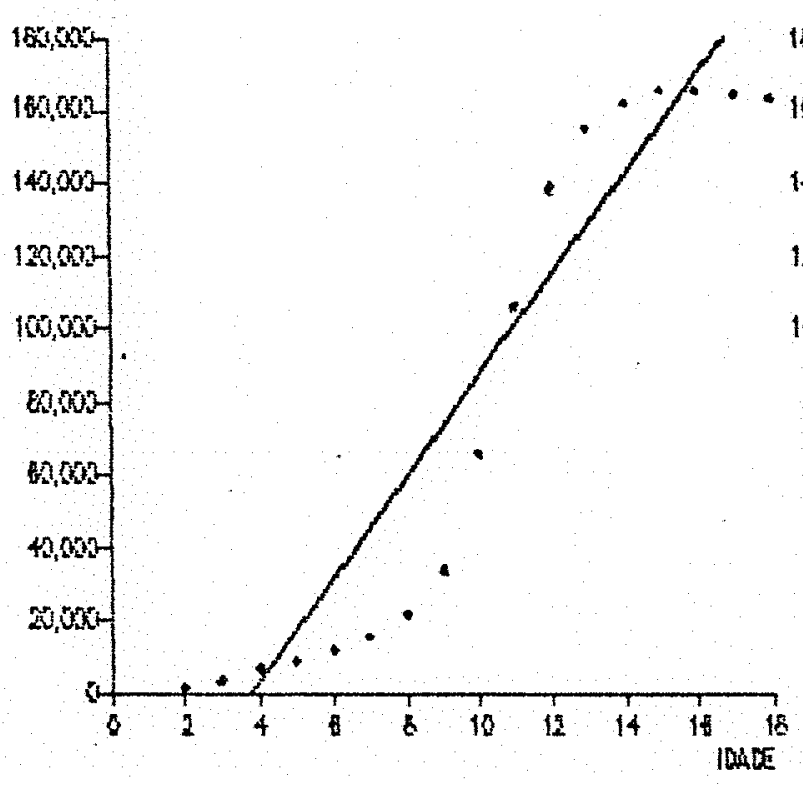

FAntin

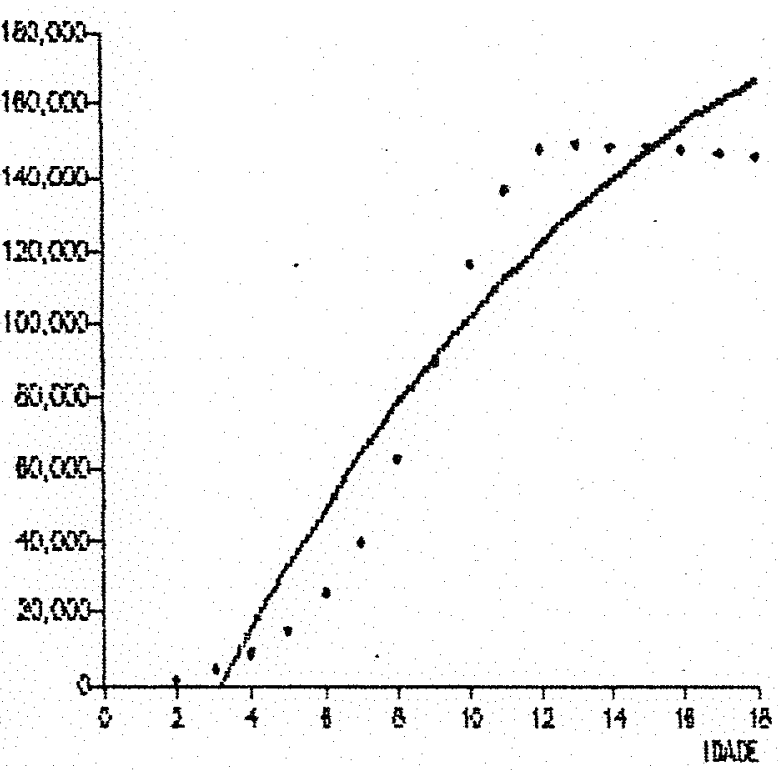

EXPERIMENTO 8

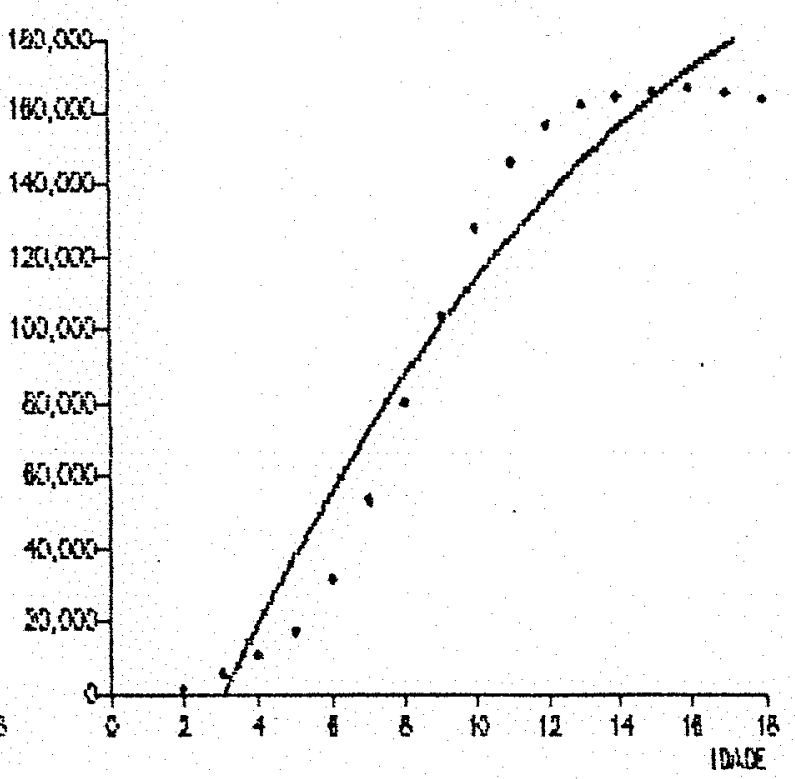

Figura 15 - Graficos das curvas estimadas atravos da funçăo Frody sobrepostos aos pontos observados nos fxper i mentos $5,6,7,8$. 
50.

0 Guadro 30 apresenta os resultados dos ajustes: da funçä logistica modificada.

QUADRO 30 - Fumgấ loggi stica Modificada

$$
\text { chi } y=\beta_{0}\left(1+e^{-\beta_{1} x_{5}-\beta_{z}}\right.
$$

\begin{tabular}{|c|c|c|c|c|c|c|}
\hline Fxp. & $\begin{array}{c}\text { Fst i mati } \\
\hat{\beta}_{0}\end{array}$ & vas dos: & $\begin{array}{c}\text { paremelros } \\
\qquad \beta_{2}\end{array}$ & gMk & $R^{2}$ & $\mathrm{u}$ \\
\hline 3 & $151 \mathrm{A6S}$ & 0,329 & 30,650 & $1.598 \times 10^{8}$ & 0,9543 & 48 \\
\hline 4 & 24357 & 0,370 & $30,8 A O$ & $8.061 \times 10^{7}$ & 0,0770 & 40 \\
\hline 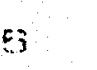 & $1 E_{55321}$ & $0,4 \& 3$ & 97,756 & $1,4053 \times 10^{8}$ & 0.9703 & $P 4$ \\
\hline 6 & $15515 \%$ & 0,150 & 30,840 & $4.734 \times 10^{7}$ & 0,080 & $10 \%$ \\
\hline$\gamma$ & 188119 & 0,354 & 30,830 & $1,3-5 \times 10^{8}$ & 0,0774 & 30 \\
\hline 8 & $1716 S A O$ & 0,437 & 24,043 & $1.872 \times 10^{7}$ & 0,0963 & 11.24 \\
\hline 9 & 151112 & 0,309 & 30,850 & $4,20 \approx \times 10^{7}$ & $0,989 \%$ & 38 \\
\hline
\end{tabular}

A funça logistica modificada a justou-se bem en todos os experimentos. o ajuste foi ligeiramente molnor ros experimentos de crescimento majs lonto, Experimentos $C 4 ; 6$; 83 . As estimativas des $\beta_{0}$ sî́c wuperiores aos valores observados de produca maxima em cacte experimento os graticos de fungho logitios

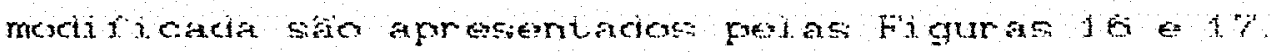




\section{EXPERIMENTO 1}

Prowed

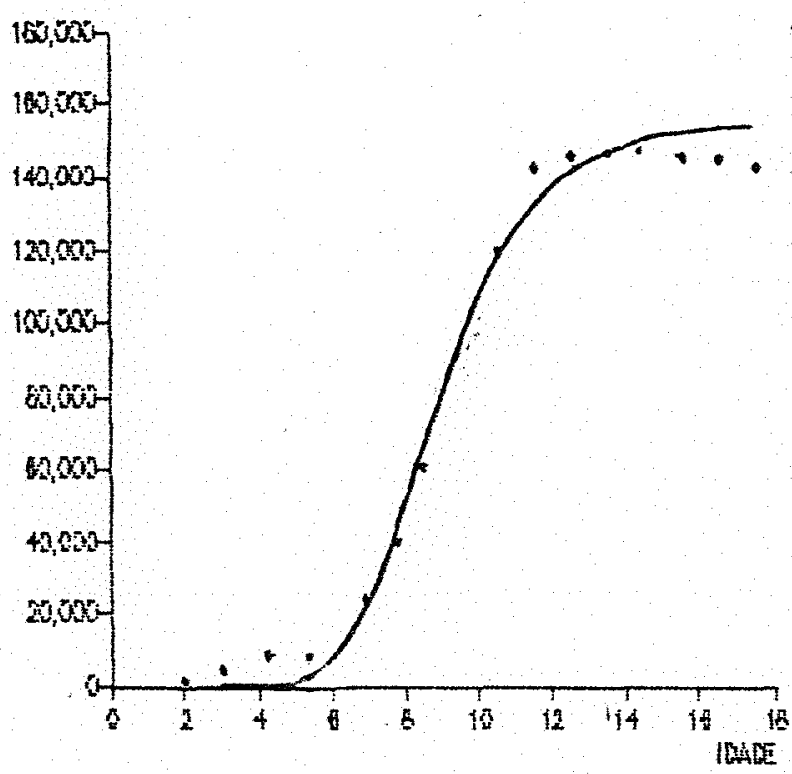

\section{EXPERIMENTO 3}

Prancan

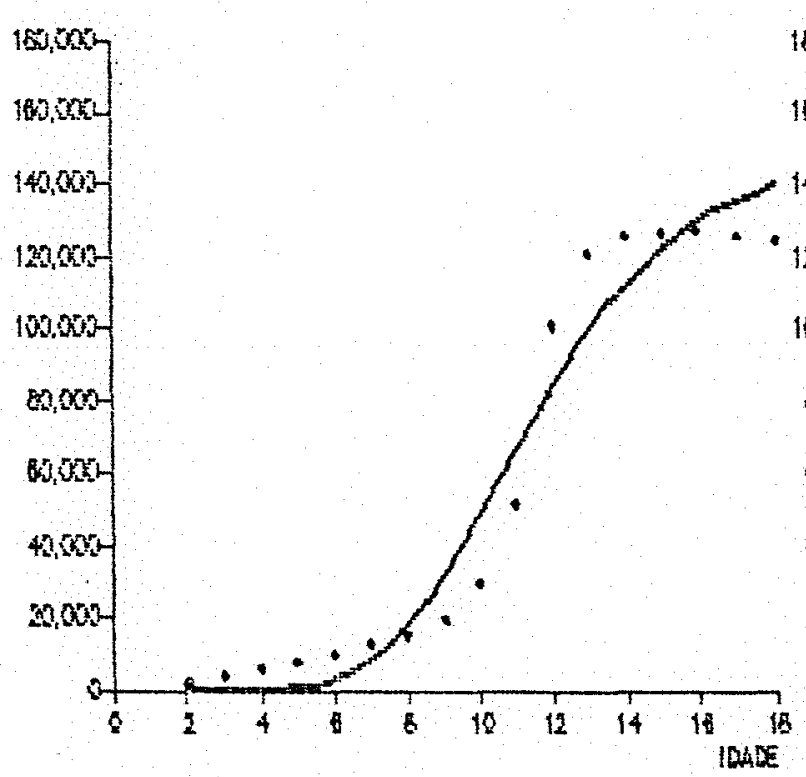

\section{EXPERIMENTO 9}

moystan

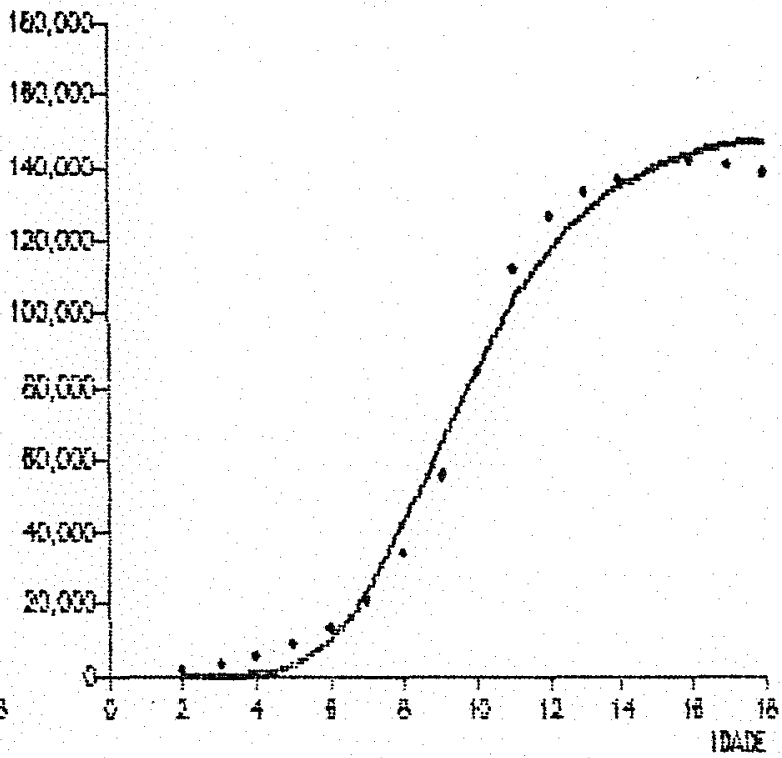

Finnent?

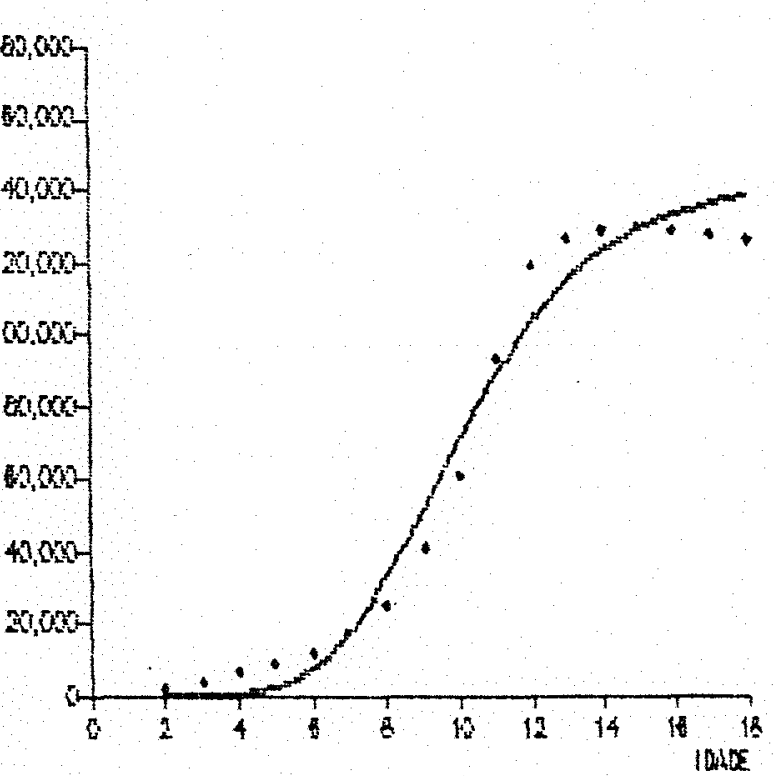

Figura 16 - Graficos das curvas ostimacias atraves da funcão Jogistica modificada sobrepostos aos poritos observados nos Experi meritos $1,0,3,4$. 


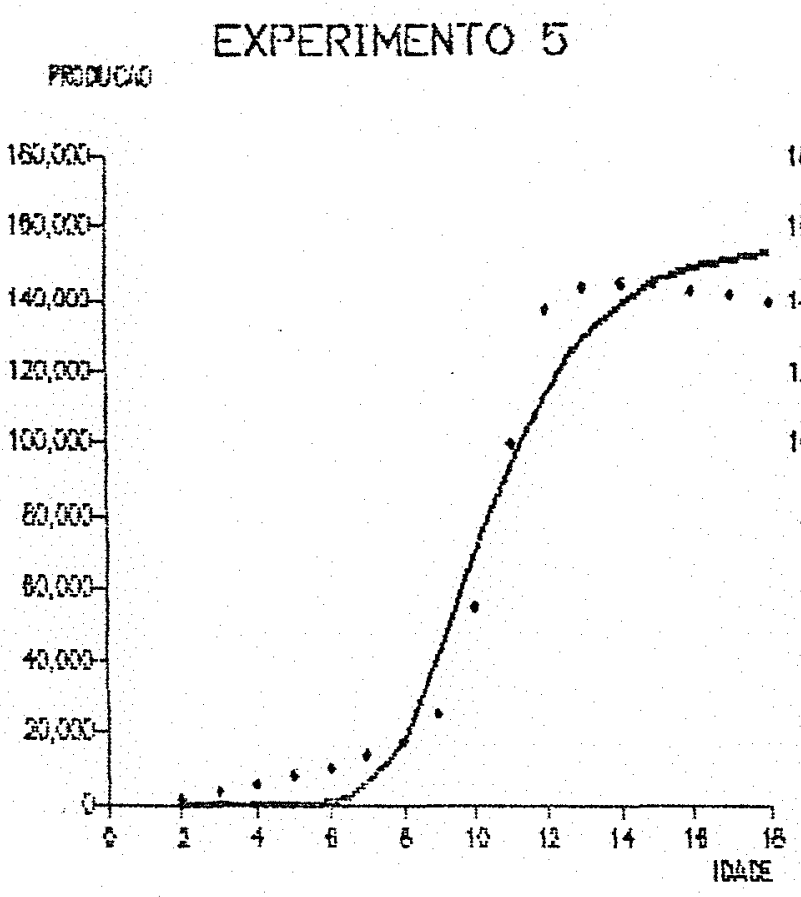

EXPERIMENTO 7

Panitio

\section{PROULA}

\section{EXPERIMENTO 6}

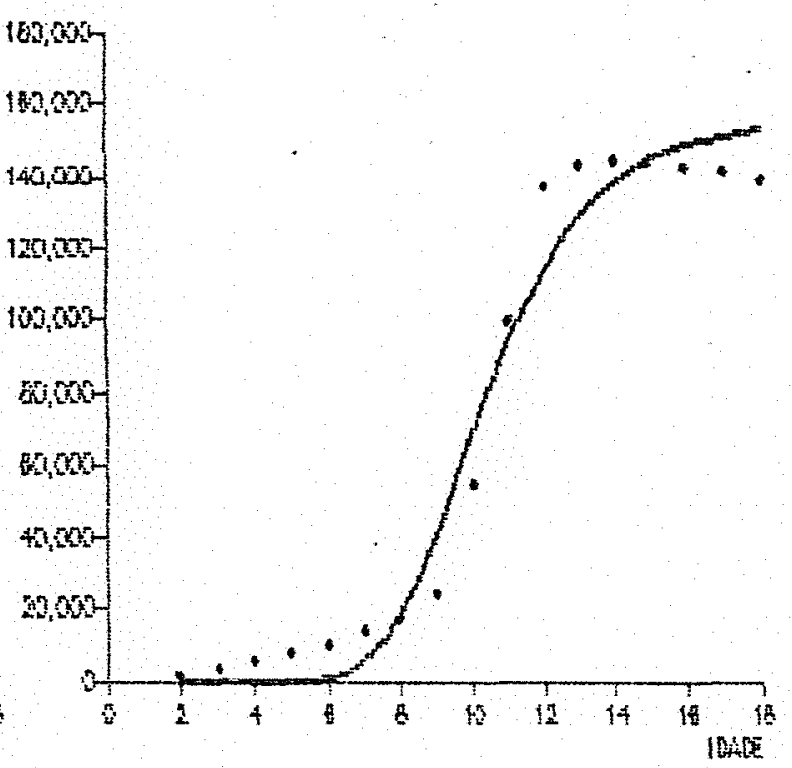

EXPERIMENTO 8

Frats
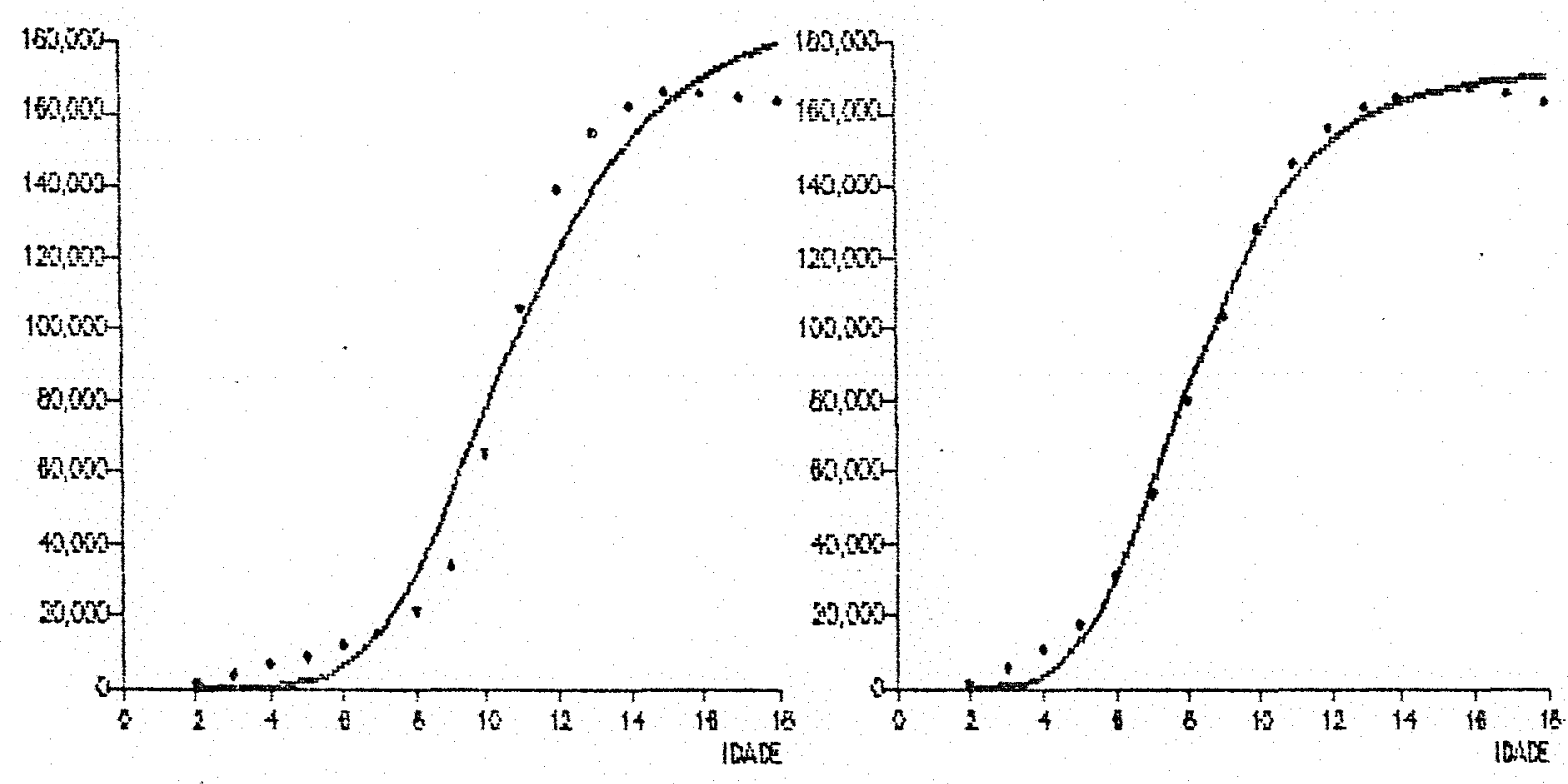

Figura 17 - Gráficos das curvas cstijmadas atraves da funcro logistica modificada sobrepostor aos fontos observados nos Experimeritos $5,6,7,8$. 
62.

No Quadro 31, estão os resultados dos ajustes da função exponencial.

\begin{tabular}{|c|c|c|c|c|c|}
\hline Exp. & $\begin{array}{l}\text { Estimati vas } \\
\hat{\beta}_{0}\end{array}$ & $\begin{array}{c}\text { dos parametro } \\
\hat{\beta}_{1}\end{array}$ & os QMR & $R^{2}$ & $\mathrm{u}$ \\
\hline 3 & 13,224 & 23,605 & $2,841 \times 10^{8}$ & 0.9126 & 7 \\
\hline 4 & 12,902 & 17.713 & $2,302 \times 10^{\mathrm{a}}$ & 0,9295 & 6 \\
\hline 5 & 13,142 & 19,843 & $4,574 \times 10^{\circ}$ & 0,8963 & 6 \\
\hline 6 & 12,718 & 12,093 & $2,751 \times 10^{\circ}$ & 0,9324 & 7 \\
\hline 7 & 13,316 & 20,589 & $3,717 \times 10^{\circ}$ & 0.9321 & 6 \\
\hline 8 & 12,793 & 11,674 & $2,170 \times 10^{8}$ & 0,9545 & 7 \\
\hline 9 & 13,142 & 19,843 & $4,574 \times 10^{8}$ & 0,8790 & 6 \\
\hline
\end{tabular}

A funçăo exponencial atingiu a convergencia em poucas i teraçóes. Embora esta seja uma função sigmóide, ela náo se apresentou eficiente no ajuste aos dados dos experimentos. A mudariça dos valores iniciais não altera as estimativas dos parametros. For exemplo, as estimativas do Experimento 8, que apresentou um melhor ajuste, săo as mesmas do Quadro 31 quando se atribuem outros valores iniciais aos parametros. Pelas figuras 18 e 10 pode-se observar a falta de ajuste da função exponencial aos dados. 


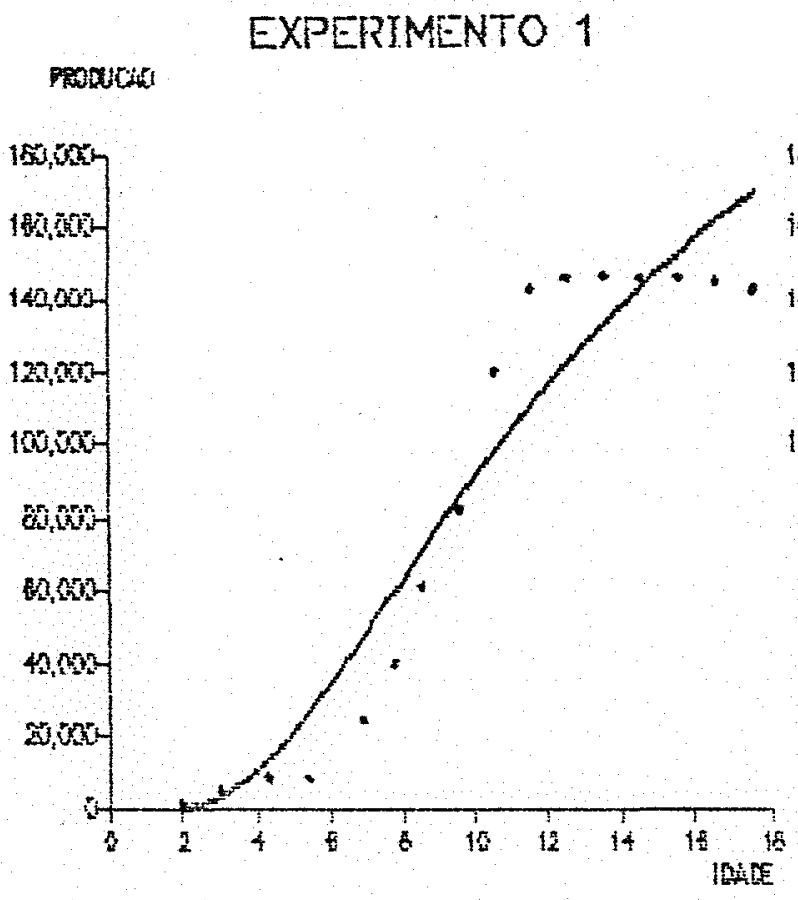

EXPERIMENTO 3

Pavoutit

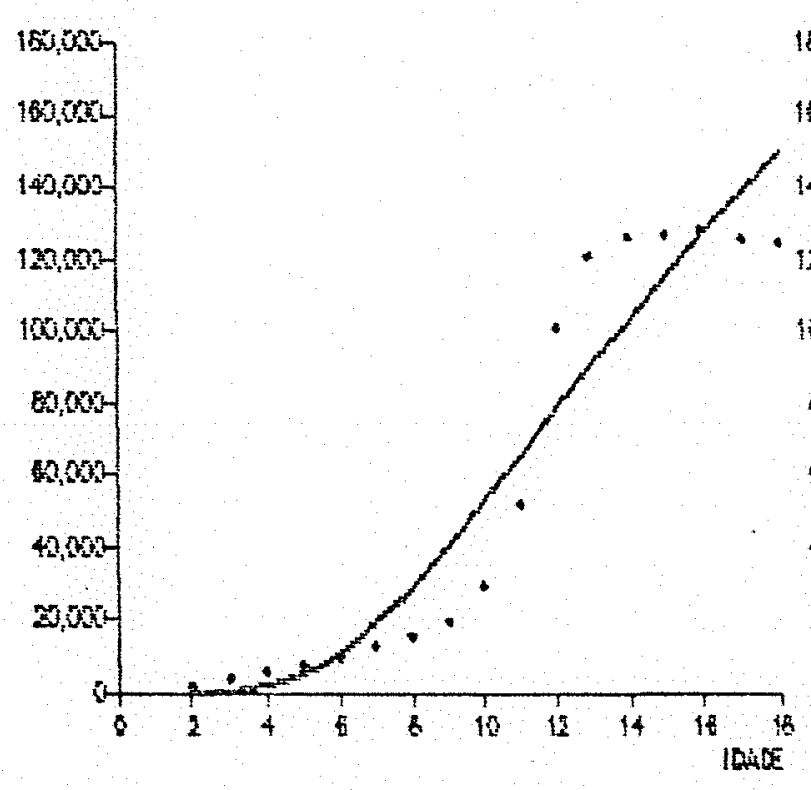

\section{EXPERIMENTO 9}

Fortan

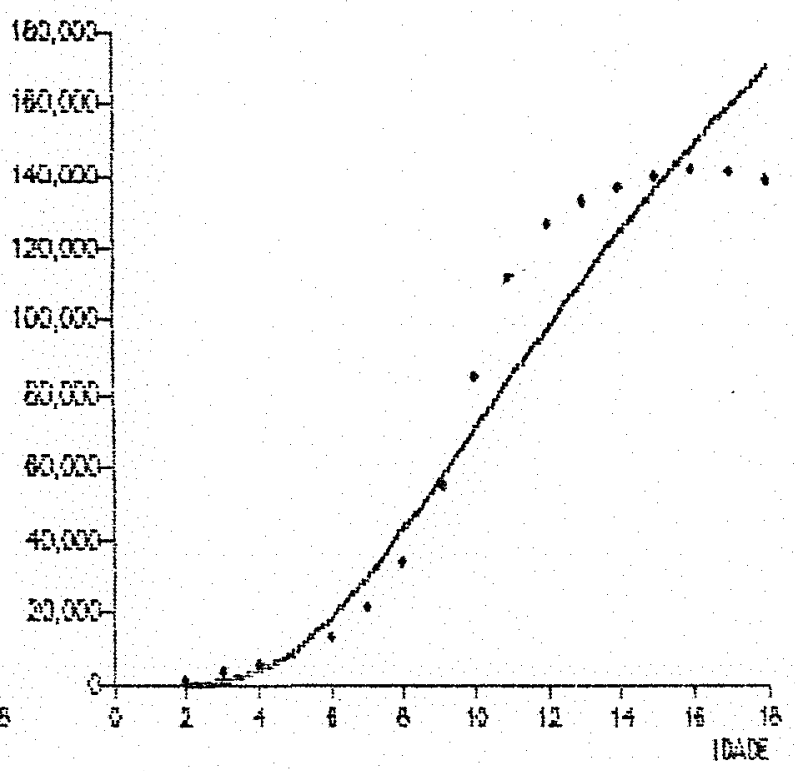

Panterin

\section{EXPERIMENTO 4}

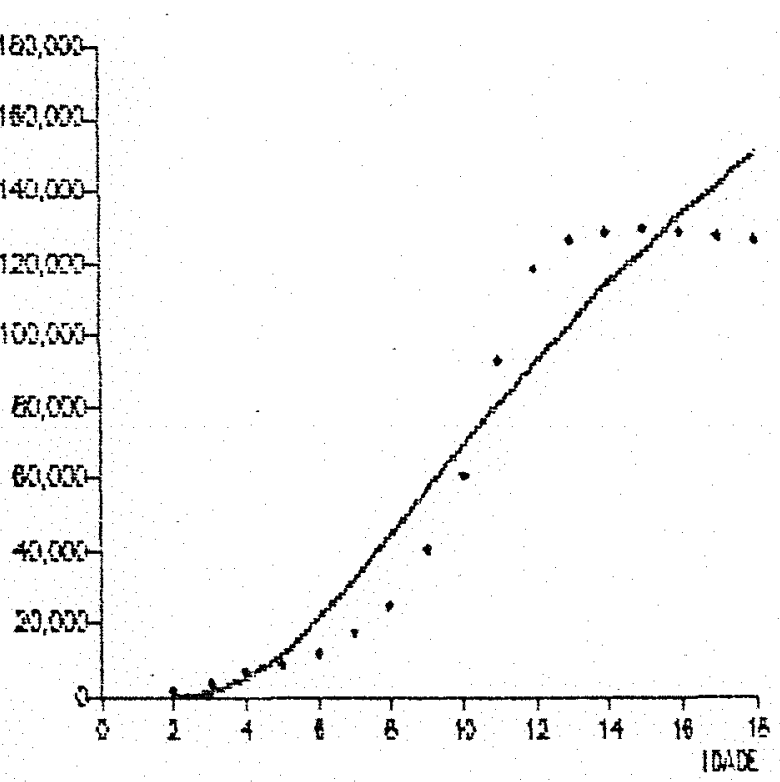

Hojura $1 \varepsilon$ - Graficos das curvas estimadas atraves da funçio exponencial sobrepostos aos pontos observacios nos Fixperimentos $1,9,3,4$. 
EXPERIMENTO 5

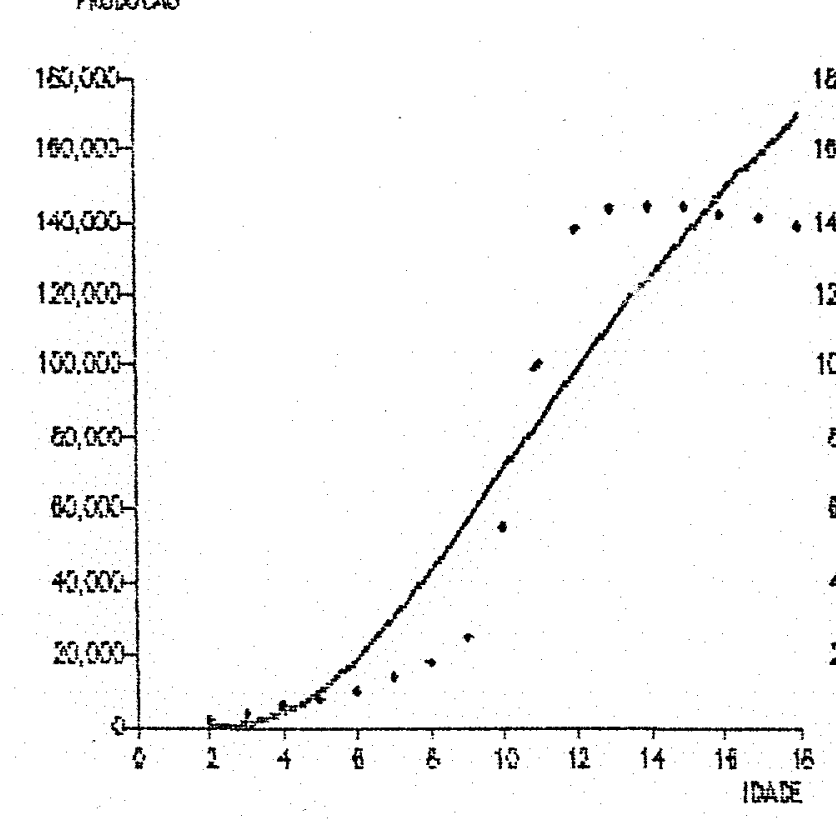

$\max$

EXPERIMENTO 6

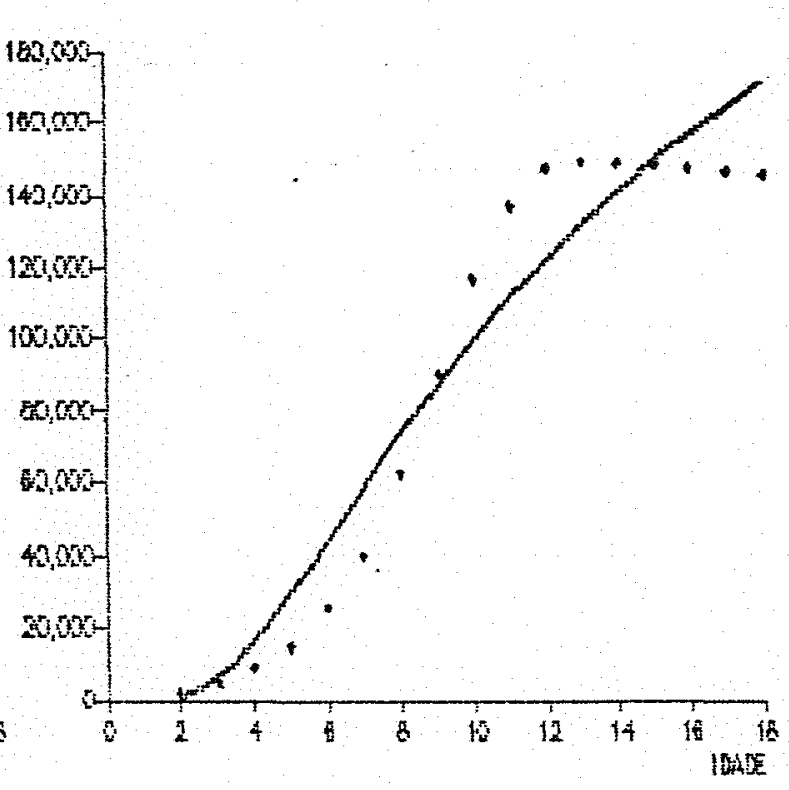

\section{EXPERTMENTO 7}

Poureverit

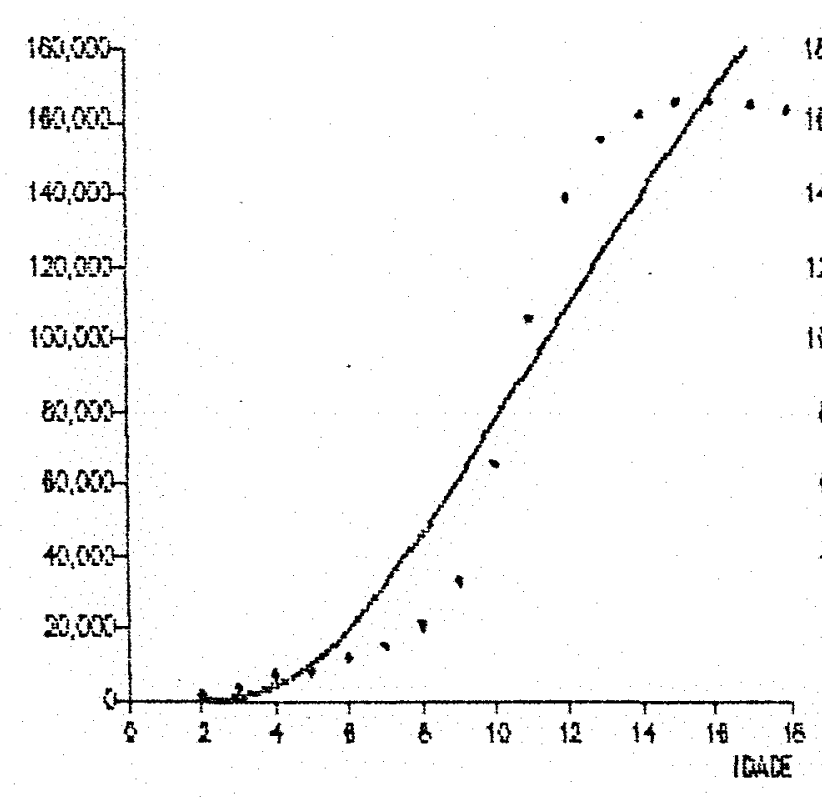

pingerting

\section{EXPERIMENTO 8}

Praten 
65.

O. resultados dos ajustes da funça log-inversa-gener aj izada saio apresentados no Quadro 32 .

QUADRO 3E - Funça Log-inversa-generai izada

(j) $y=\beta_{0}+\beta_{1} \beta_{2}^{1}$

\begin{tabular}{|c|c|c|c|c|c|c|}
\hline Exp. & $\begin{array}{c}\text { Est i mat } \\
\hat{\beta}_{a}\end{array}$ & $\begin{array}{c}\text { divas dos } \\
\beta_{1}\end{array}$ & $\begin{array}{c}\text { parametros } \\
\beta_{z}\end{array}$ & QMR & $E^{2}$ & $\mathrm{u}$ \\
\hline 3 & $-158 \%$ & 543546 & $\Omega_{,} 240 \times 10^{-12}$ & $3,036 \times 10^{8}$ & 0,9129 & 28 \\
\hline 4 & -3004 & 390856 & $4.870 \times 10^{-8}$ & $2,431 \times 10^{8}$ & 0,9305 & 12 \\
\hline 5 & -5514 & 401390 & $7.890 \times 10^{-9}$ & $4.802 \times 10^{8}$ & 0.8979 & 30 \\
\hline 8 & -10303 & 328104 & $1,970 \times 10^{-5}$ & $2,797 \times 10^{8}$ & 0.9359 & 13 \\
\hline 7 & -4971 & 5836701 & $3,150 \times 10^{-0}$ & $3,916 \times 10^{8}$ & 0.9332 & 37 \\
\hline 8 & -10043 & 354411 & $=760 \times 10^{-5}$ & $2.169 \times 10^{8}$ & 0,9576 & 8 \\
\hline 0 & -6060 & 386418 & $P, 940 \times 10^{-7}$ & $2.217 \times 10^{8}$ & 0.9453 & 11 \\
\hline
\end{tabular}

Com caracterjsticas semelhantes a funçăo exponencial, a fungto loo-inversa-generalizada náa se ajustion bem aos dados dos experimentos. A mudanga dos valores incials na melhora o djuste A assintota superior dada por $y=\hat{\beta}_{\alpha}+\hat{\beta}_{1}$, resulta num valor mulo alto em todos os experimentos o ponto de lnîjexăo e dado pelas coordenadas $x=-1 \mathrm{n} \beta_{2} / \theta, y=\hat{\beta}_{0}+\hat{\beta}_{1} \rho$ Nos exper mentos com orescimento mal s lento C4; $6 ; 8$; 8 , o ponto de unflexto ncontece para valores balxos de $x$ $C \times=6,4$ ro Exp Ej Os graficos da funcão log-inversa-generalizada estao nas figuras 20 e 
EXPERIMENTO 1

Pondod

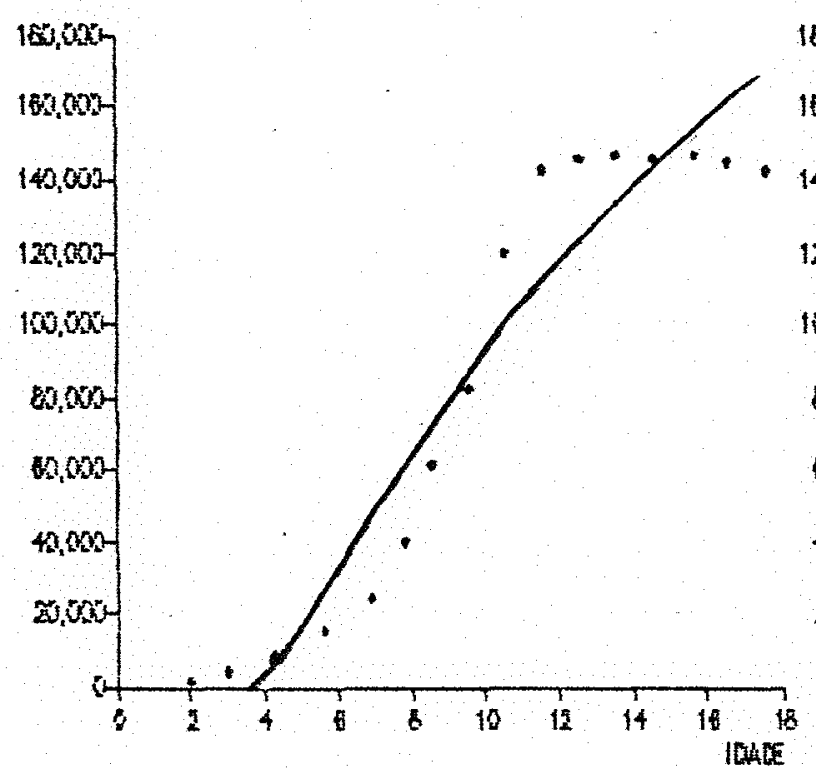

EXPERIMENTO 3

Paxturin

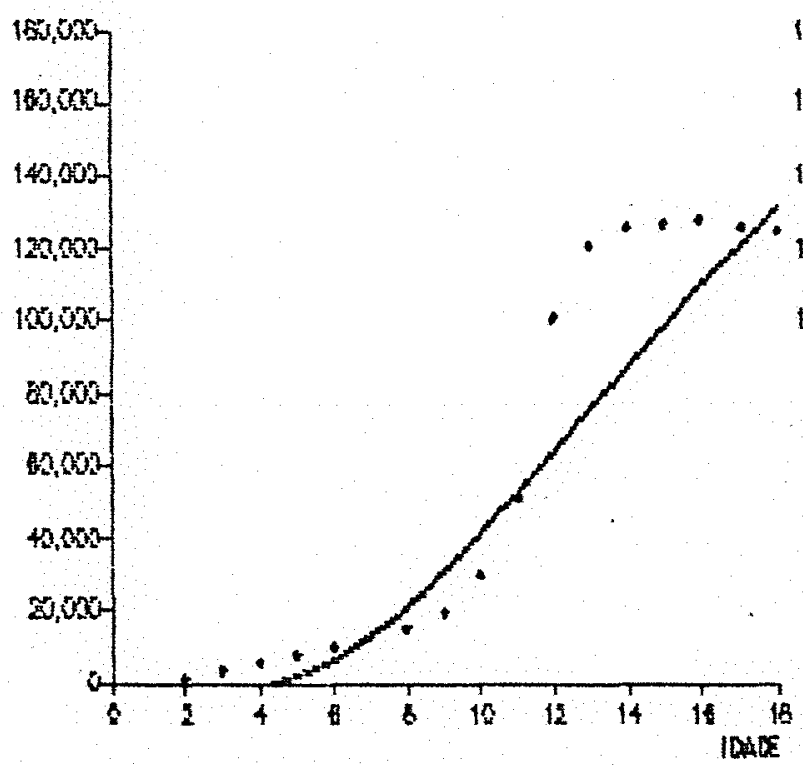

EXPERIMENTO 9

Fanding

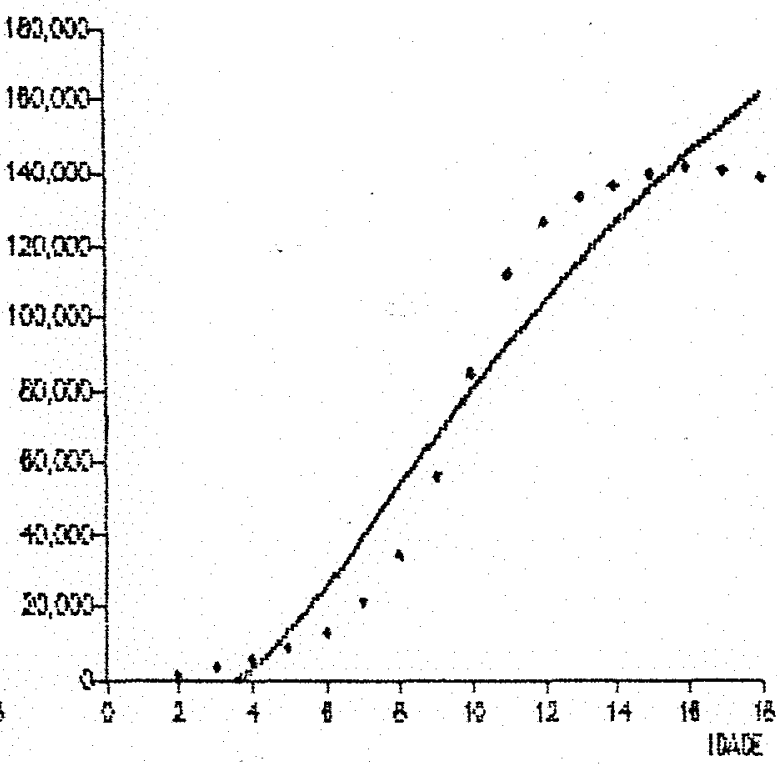

EXPERIMENTO 4

Pantin

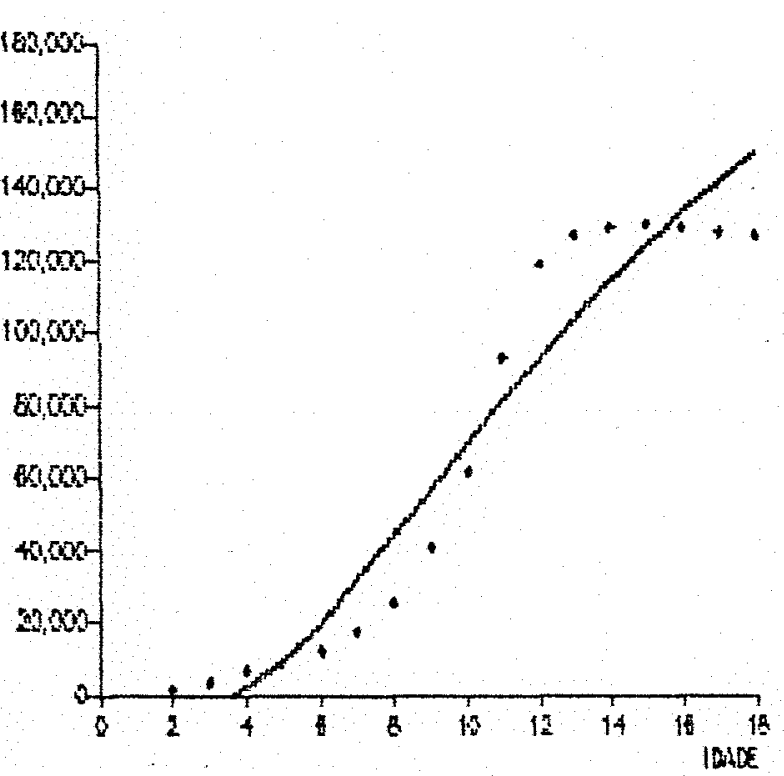

Figura 20 - Graficos das curvas ostimadas atraves da funç̄o log-j nversa-general i zada sobrepostos aos pontos: observados nos Experimentos: 1. $0,3,4$. 


\section{EXPERIMENTO 5}

Pancito

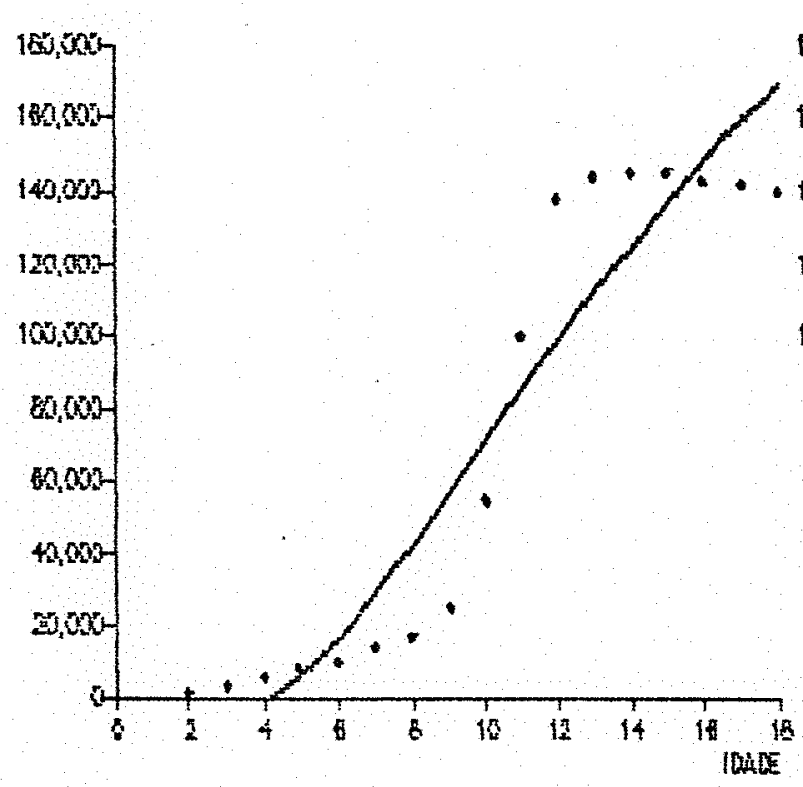

EXPERIMENTO 7

Fonowar prowin

EXPERIMENTO 6

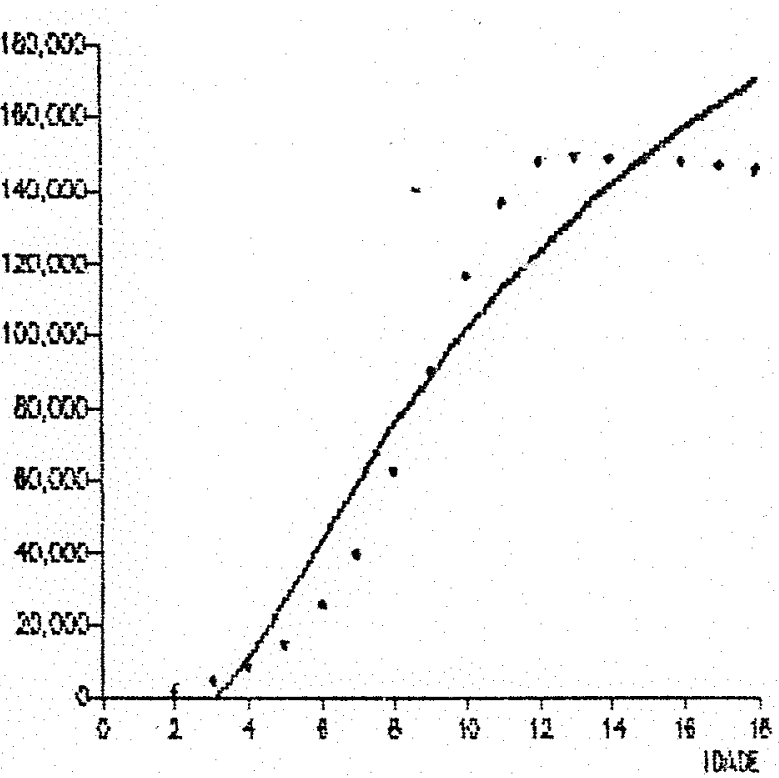

\section{EXPERIMENTO 8}

Fnounin
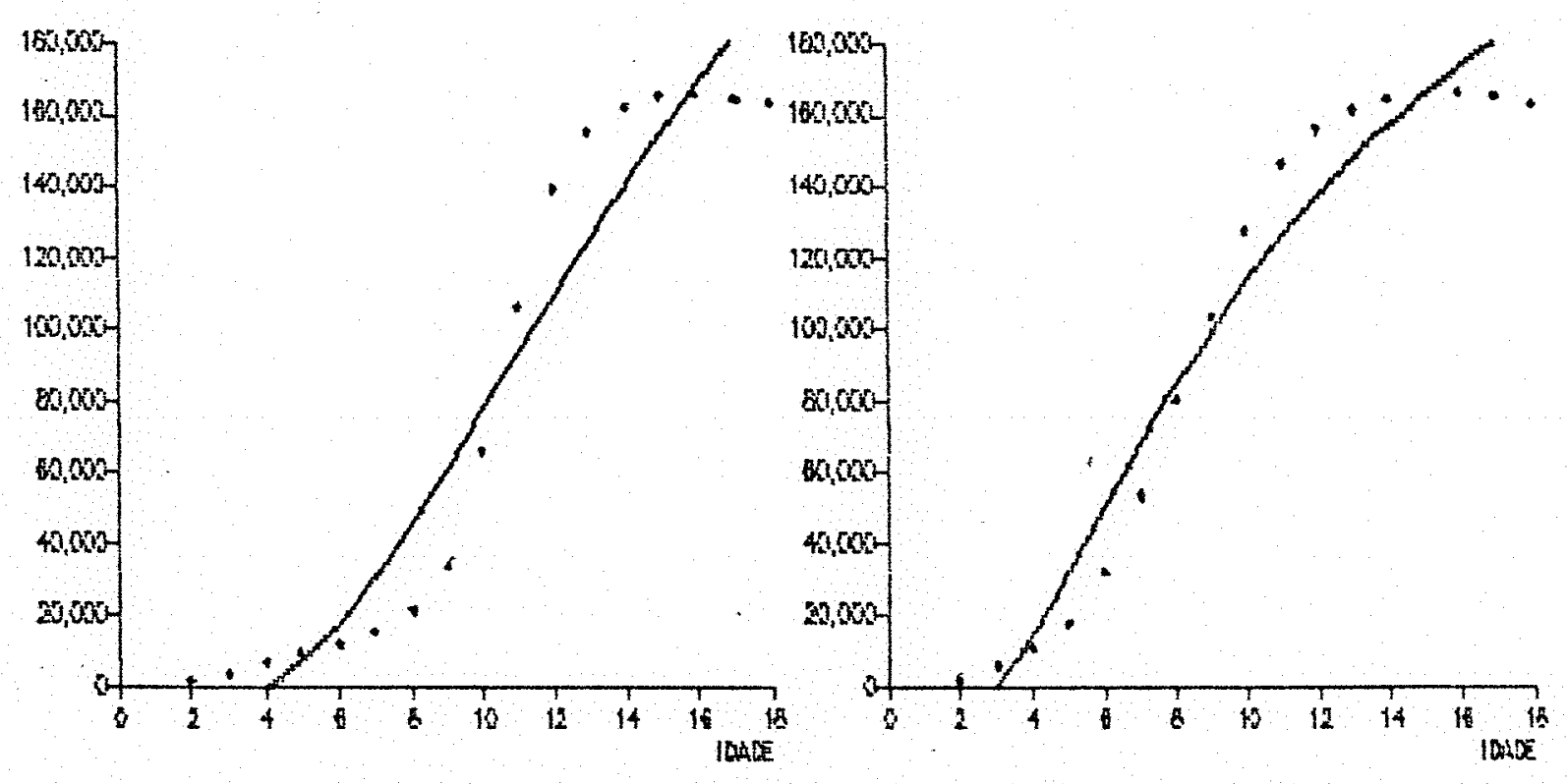

Figura oj - oraricos das curvas estimadas atraves da runs:̃o log-inversa-general i zada sobrepostos hos pontos observados nos Experimentos $5,6,7,8$ 
68.

No Quadro 33 , estáo os resultados dos ajuster da funcio de Richards.

QuAnko 33 - Funça de kichards $(k)$ y $=\beta_{0}\left(1+\beta_{1} e^{-\beta_{2}} x^{-1} / \beta_{3}\right.$

\begin{tabular}{|c|c|c|c|c|c|c|c|}
\hline \multirow[t]{2}{*}{ Exp } & \multicolumn{2}{|c|}{ Fst, imati vas dos } & \multicolumn{2}{|c|}{ partametros } & \multirow[t]{2}{*}{ QMR } & \multirow[t]{2}{*}{$\mathrm{R}^{2}$} & \multirow[t]{2}{*}{$u$} \\
\hline & $\hat{\beta}_{0}$ & $\hat{\beta}_{1}$ & $\beta_{2}$ & $\beta_{3}$ & & & \\
\hline 3 & 125834 & $4,077 \times 10^{25}$ & 4,711 & 9,237 & $1.820 \times 10^{7}$ & 0,9951 & 785 \\
\hline 4 & 128500 & $P .479 \times 10^{1 \Xi}$ & 2,431 & 5,819 & $1,372 \times 10^{6}$ & 0.9996 & 376 \\
\hline 5 & 143003 & $3,513 \times 10^{20}$ & 4,060 & 7,145 & $1.177 \times 10^{7}$ & 0,9977 & 800 \\
\hline 6 & 148763 & 187432 & 1,801 & 2.554 & $3,091 \times 10^{6}$ & 0,9993 & 98 \\
\hline 7 & 1634697 & 61473080 & 1,531 & 2,947 & $8,806 \times 10^{6}$ & 0,9986 & 208 \\
\hline 8 & 166095 & 320 & 0,089 & 1.110 & $1.800 \times 10^{6}$ & 0,9984 & 200 \\
\hline 9 & 140027 & 126030 & 1,104 & 2,180 & $1.509 \times 10^{6}$ & 0.9997 & 109 \\
\hline
\end{tabular}

A funçáo de Richards apresentou um excel ente ajuste em todos os experimentos, sendo ainda melhor nacfueles, cuja curva de crescimento e mais suavizada As estimativas de $B_{0}$ são ligeiramente infertores as produstres maximas observadas em cada experimento. As estj mativas de $P_{1}$ varjam sensivel mente de acordo com a inclinacia da curva, sendo malores nos experimentos que possuem a caracteristica de um crescimento mas a acentuado $(3,5,7)$ e menores naqueles de crescimentio mais suavizado $C 4,6,8,8$. Nas Fjguras ad a as observa-se atraves dos oraflcos da funço de kuchards. a eficienola desta fumcalo em ajustar-se aos dados. 


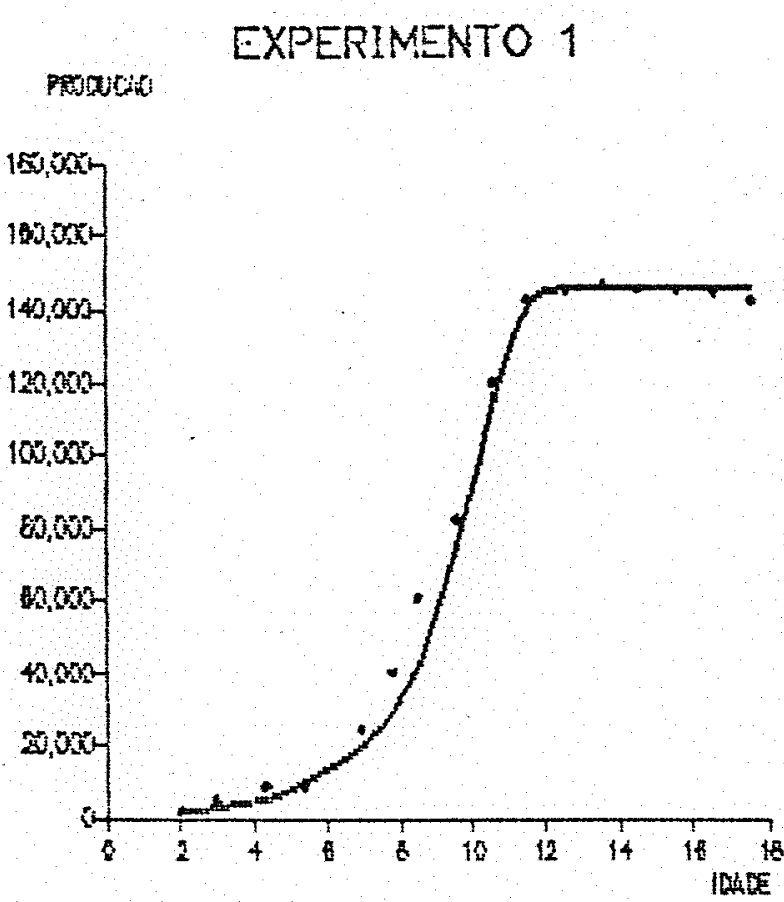

EXPERIMENTO 3

Fupuctio

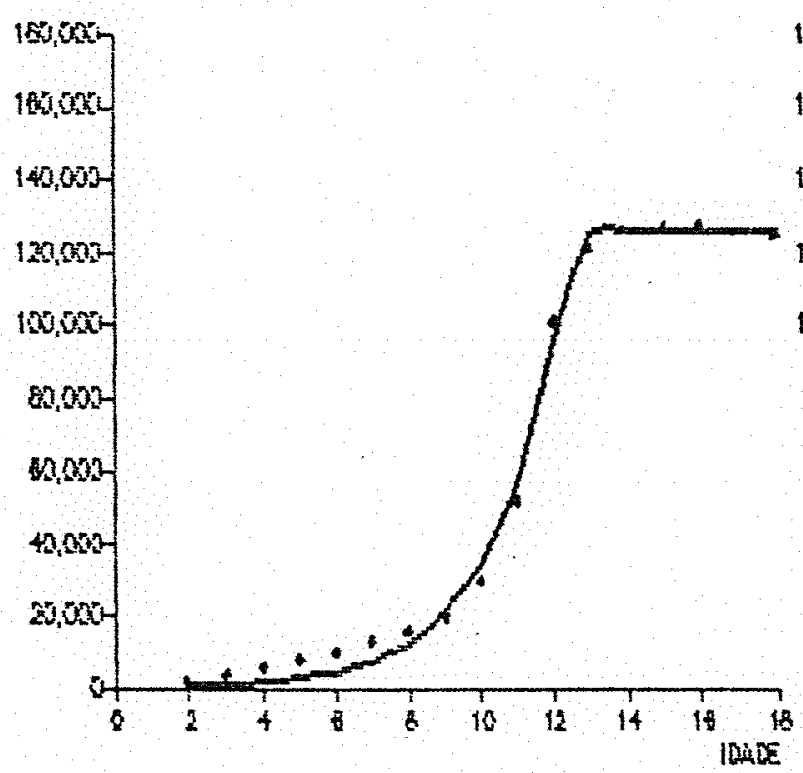

EXPERIMENTO 9

\section{pirowen}

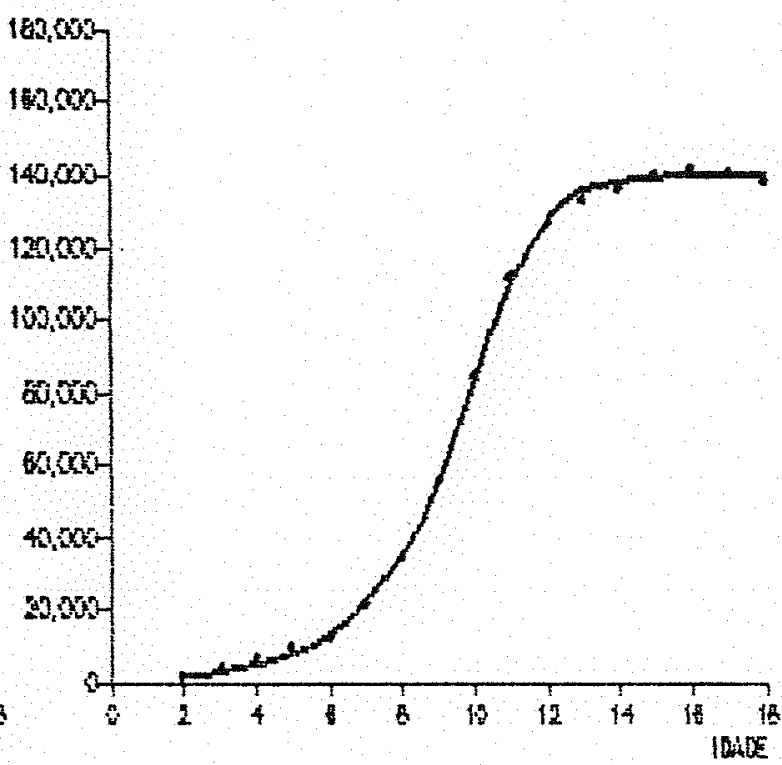

EXPERIMENTO A

FRONH

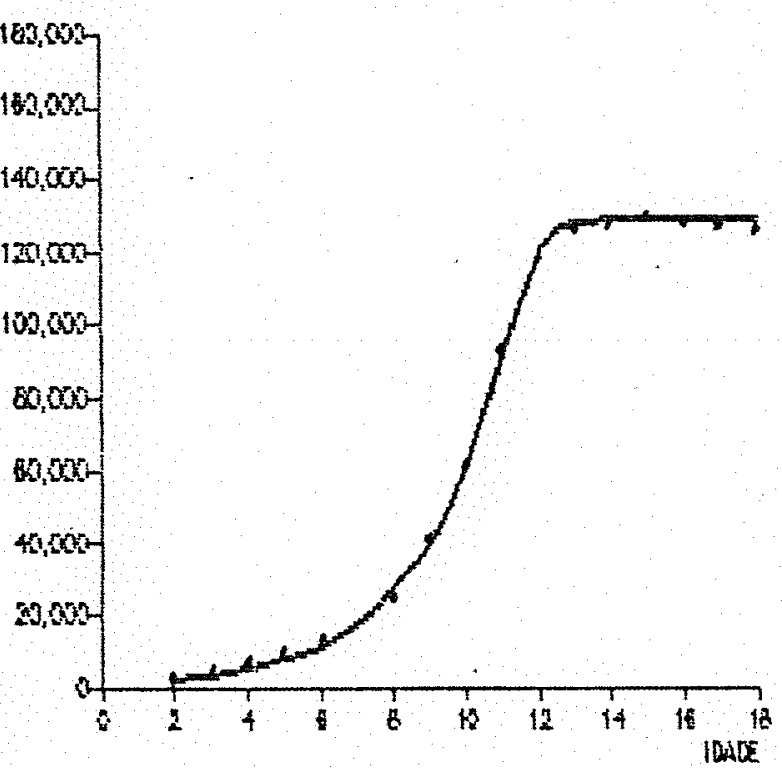

Figura es orarjeos das curvas estimadas atraves da runçio Richards sobrepostos aos pontos. observados nos Experi mentos $1,9,3,4$. 


\section{EXPERIMENTO 5}

PNowCito

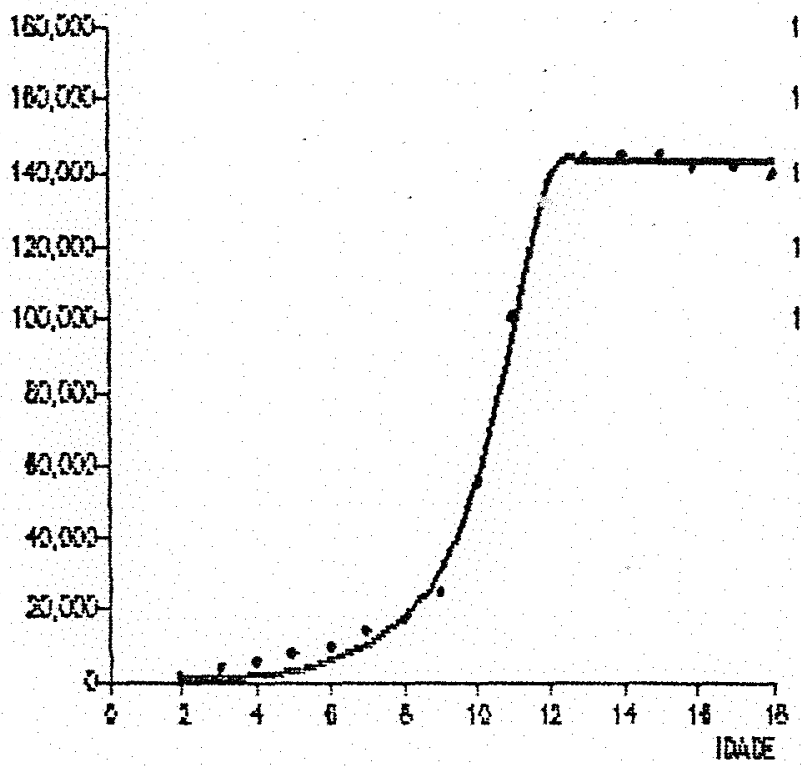

EXPERIMENTO 7

Fontidid

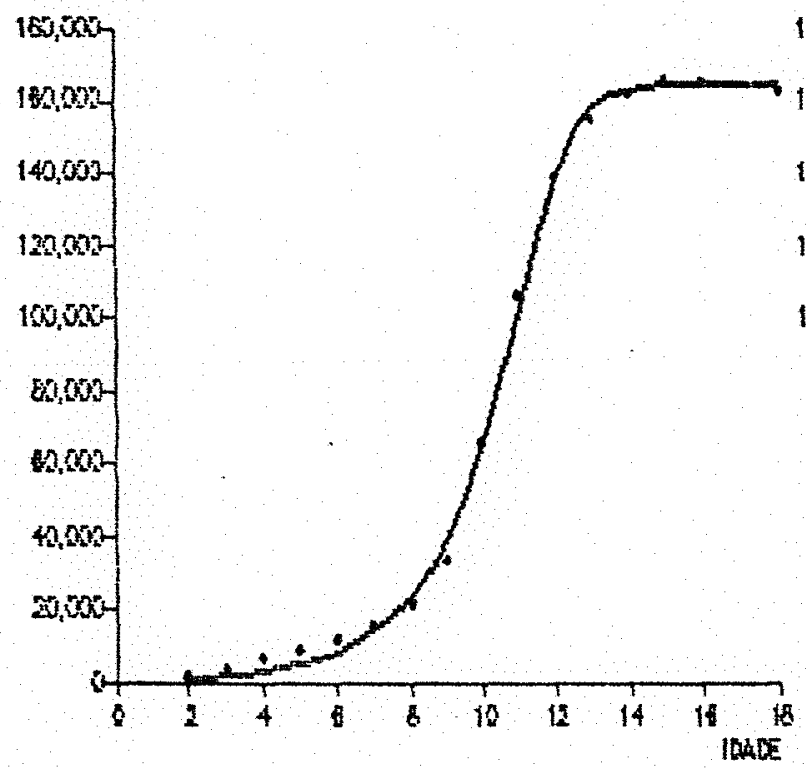

EXPERIMENTO 6

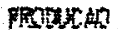

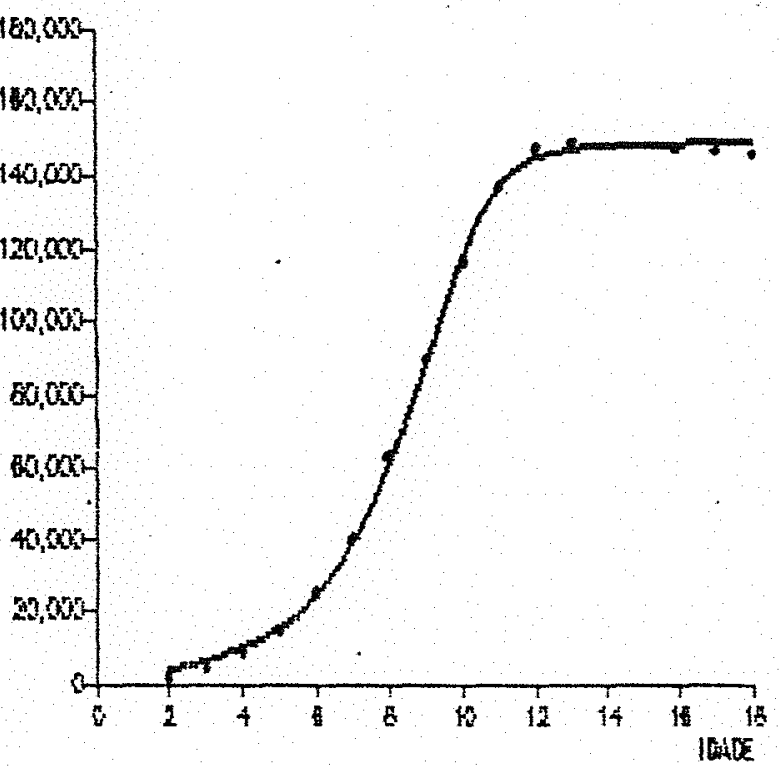

EXPERIMENTO 8

prouting

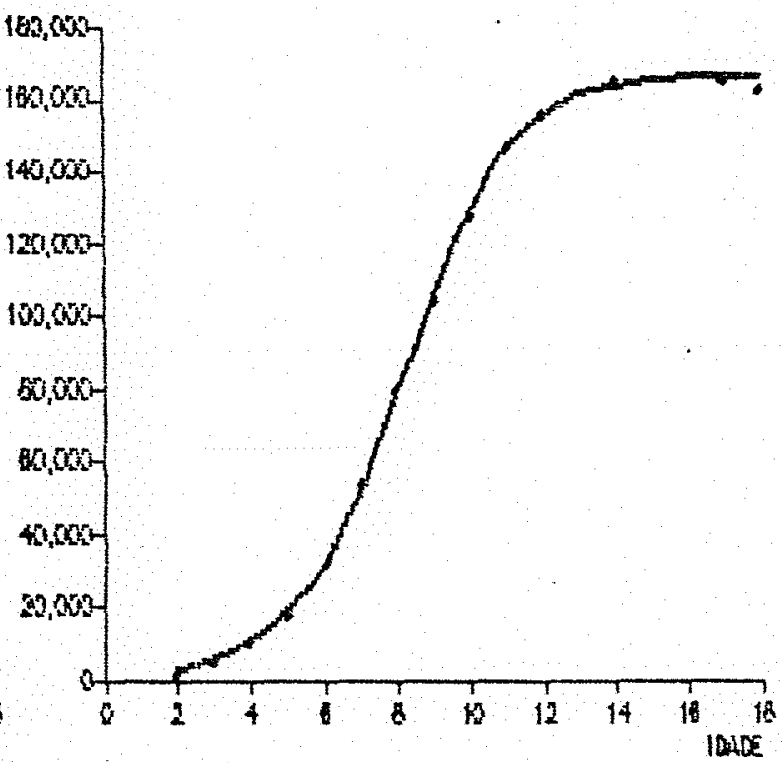

Higura 23 - Grarjeos das curvas estimadas atraves da função Richards sobrepostos aos poritos observados nos Experi mentos $5,6,7,8$. 
No Quadro 34 , estão os resultados dos ajustese da funçăo de kejd.

QUADRO 34 Funçă de Reid CIS y $=\beta_{0}+\beta_{1} e^{-\beta_{2} x^{\beta}}$

\begin{tabular}{|c|c|c|c|c|c|c|c|}
\hline \multirow[t]{2}{*}{ Exp. } & \multicolumn{4}{|c|}{ Estimativas dos parametros } & \multirow[t]{2}{*}{ QMP } & \multirow[t]{2}{*}{$R^{2}$} & \multirow[t]{2}{*}{$\mathrm{u}$} \\
\hline & $\hat{\beta}_{0}$ & $\hat{\beta}_{t}$ & $\beta_{e}$ & $\hat{\beta}_{3}$ & & & \\
\hline 3 & -16783 & $4,070 \times 10^{-14}$ & -30 & 0,035 & $4,349 \times 10^{8}$ & 0,8841 & 33 \\
\hline 4 & -33300 & $8,945 \times 10^{-14}$ & -39 & 0.025 & $3,801 \times 10^{8}$ & 0,8991 & 31 \\
\hline 5 & $-3 r 58 \%$ & $7,650 \times 10^{-14}$ & -39 & 0,028 & $0.929 \times 10^{8}$ & 0.8638 & 31 \\
\hline$\theta$ & $-115 r 01$ & $2,608 \times 10^{-13}$ & -40 & 0,012 & $4,547 \times 10^{8}$ & 0,9032 & 51 \\
\hline 7 & $-3,000$ & $6,883 \times 10^{-14}$ & -39 & 0.030 & $0,156 \times 10^{8}$ & 0,0026 & 37 \\
\hline$E$ & -132914 & $2,100 \times 10^{-13}$ & -40 & 0,012 & $3,957 \times 10^{8}$ & 0,0281 & 57 \\
\hline 9 & -40392 & $1,180 \times 10^{-13}$ & -30 & 0,020 & $3,704 \times 10^{8}$ & 0,9137 & $\overrightarrow{C_{7}}$ \\
\hline
\end{tabular}

A funç̃o de Reid năo apresentou um bom ajuste em todos os experimentos, sendo ainda pior raqueies cujo crescimento a mais rapido cexperimentos $3,5,7)$. A mudanga dos val ores iniciajs náo mej hora o ajuste.

As Figuras a e as mostram os graficos das curvas estimadas pel a functio de Fei d sobrepostas aos dados observados dos experimertos.

No Quadro 35 está os quadrados medios residuals de todas as funços ajustadas aos dados de todos os experi mentios.

No Quadro 36 se encontram os valores dos coeficientes de determactio corrojdos para os graus de 1. berdade de todas as funcres ajustadas aos dados de todos os exper i mertos. 


\section{EXPERIMENTO 1}

moucin

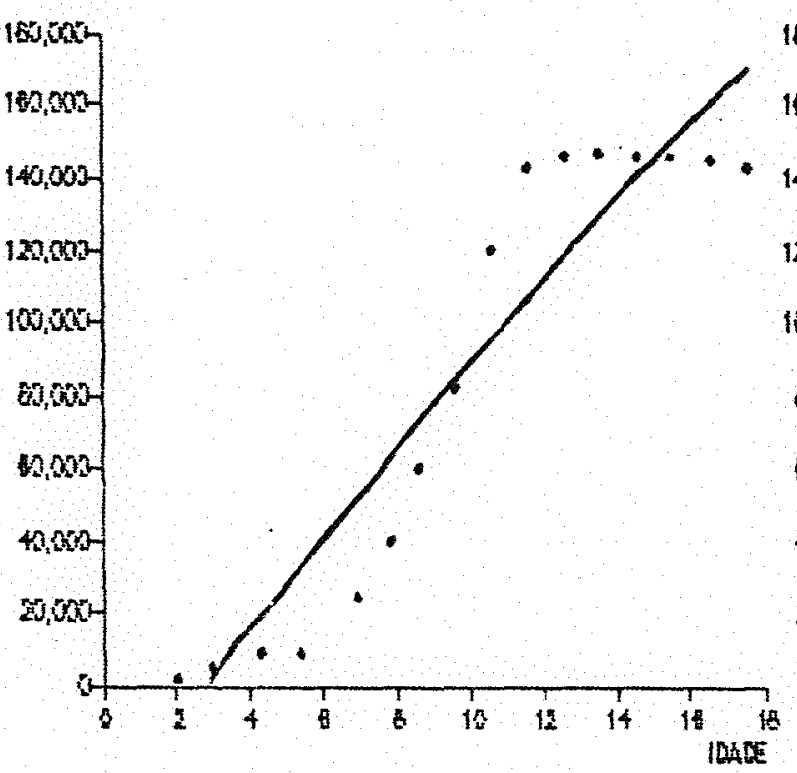

EXPERIMENTO 3

FRourif

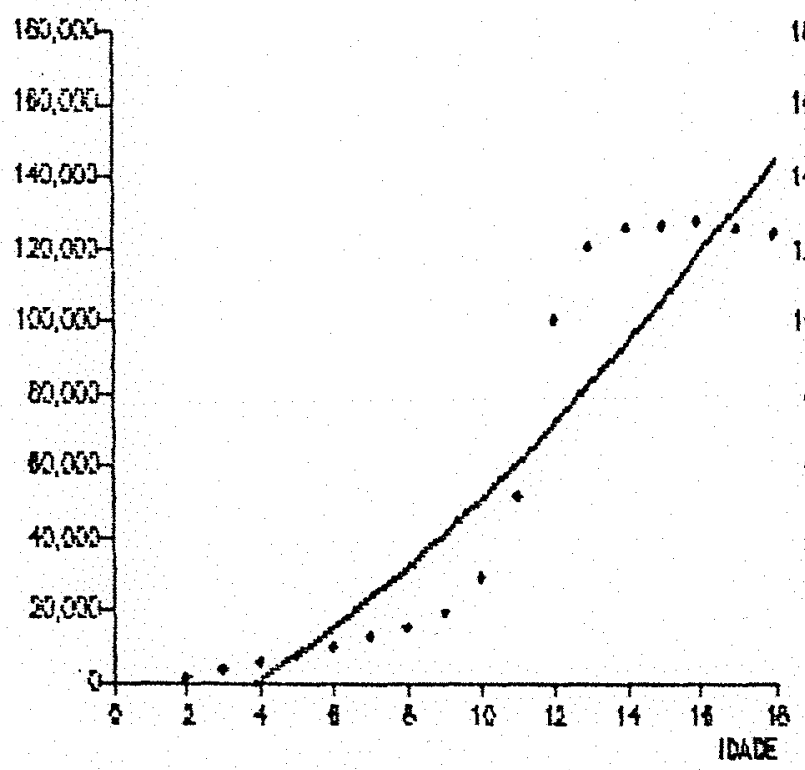

EXPERIMENTO 9

moxin

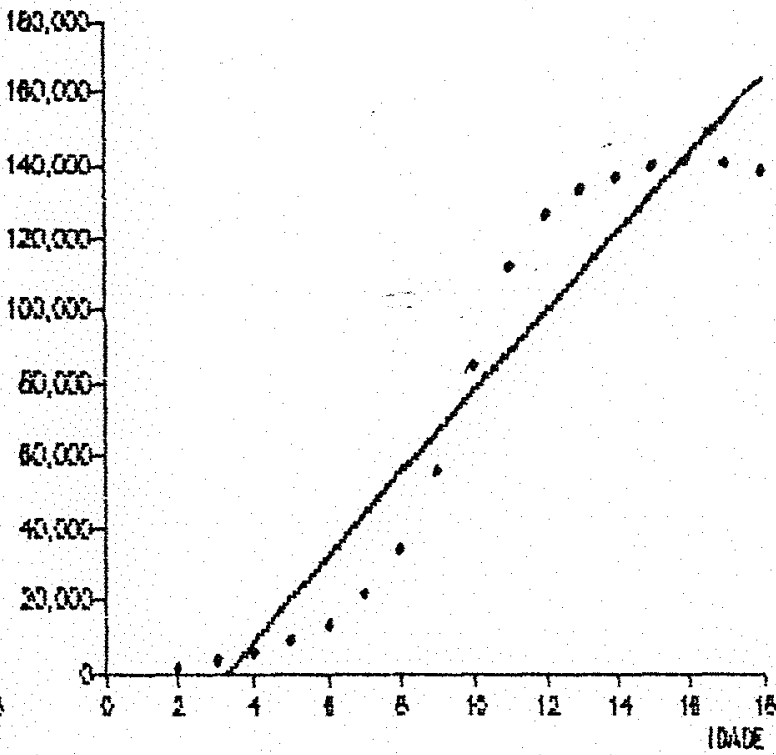

EXPERIMENTO 4

Funten

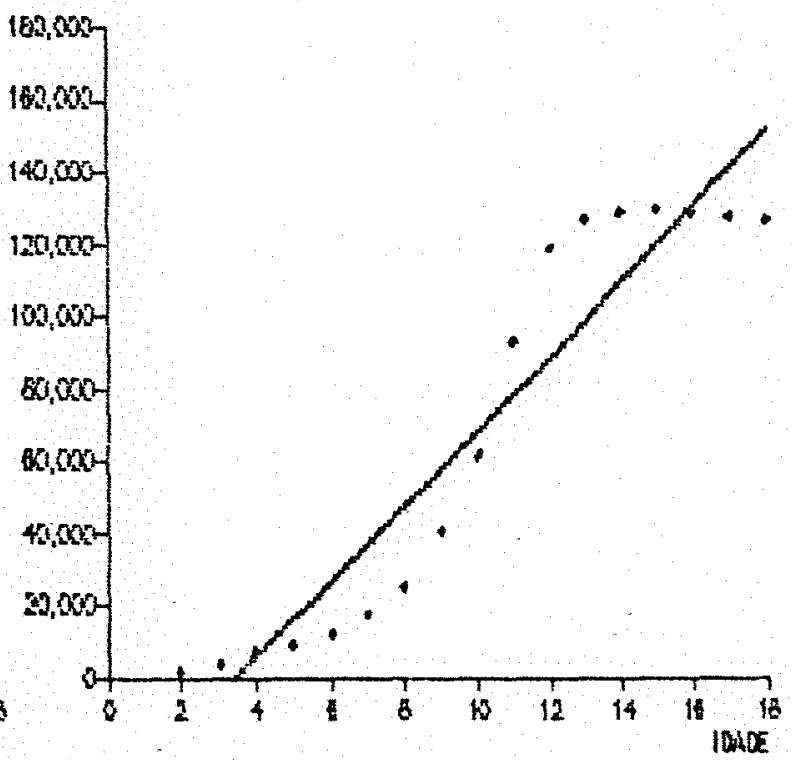

Figura 34 - Grafjcos das curvas estimadas atraves da runctio Reld sobrepostos aos pontos obsiorvados nos Feperimantoss $1,8,3,4$. 


\section{EXPERIMENTO 5}

propuction

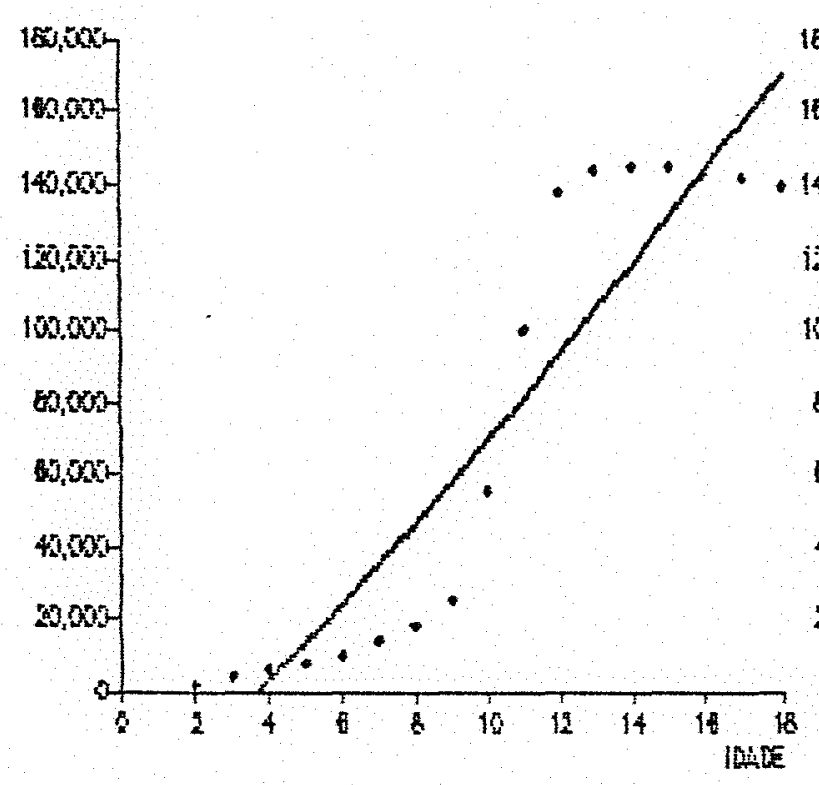

mavin

EXPERIMENTO 6

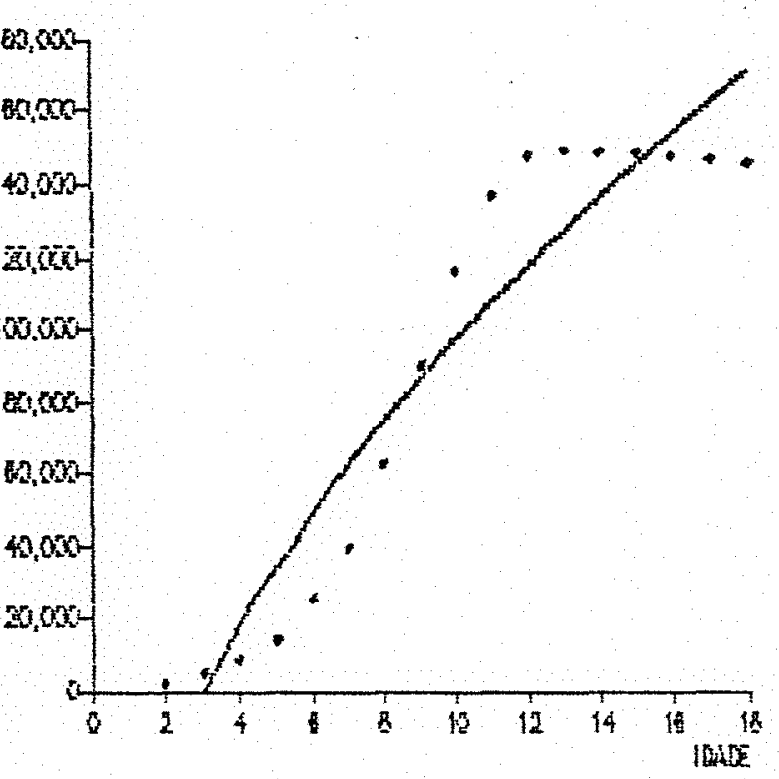

\section{EXPERIMENTO 7}

proweda

\section{prowerin}

\section{EXPERIMENTO 8}

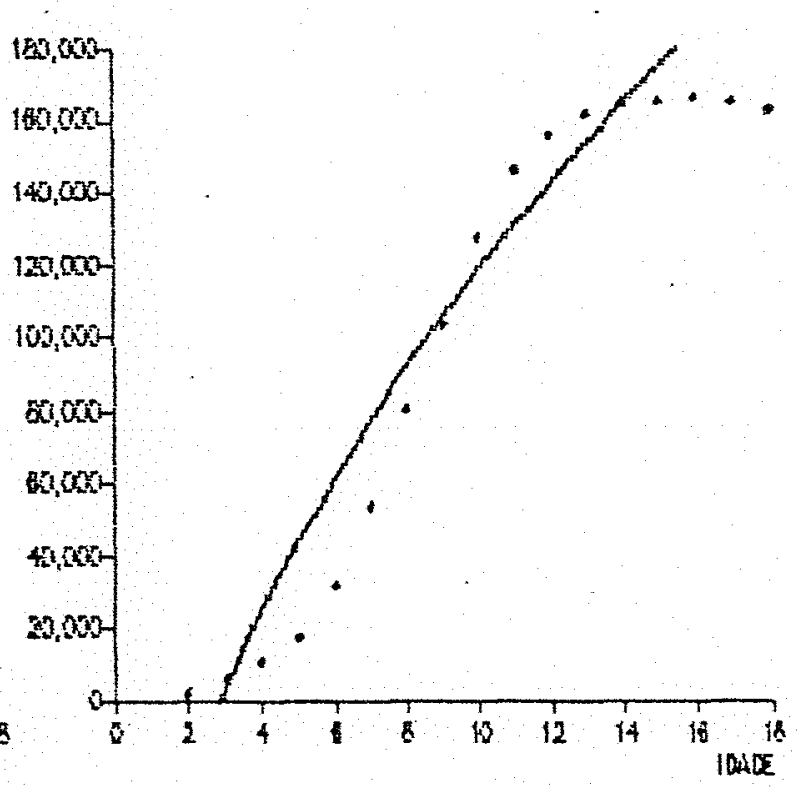

Fjgura 25 - Graficos das curvas estimadas atraves da furçäo Roid sobrepostos aes pontos observados nos: Fxperimentos $5,0,7,8$. 


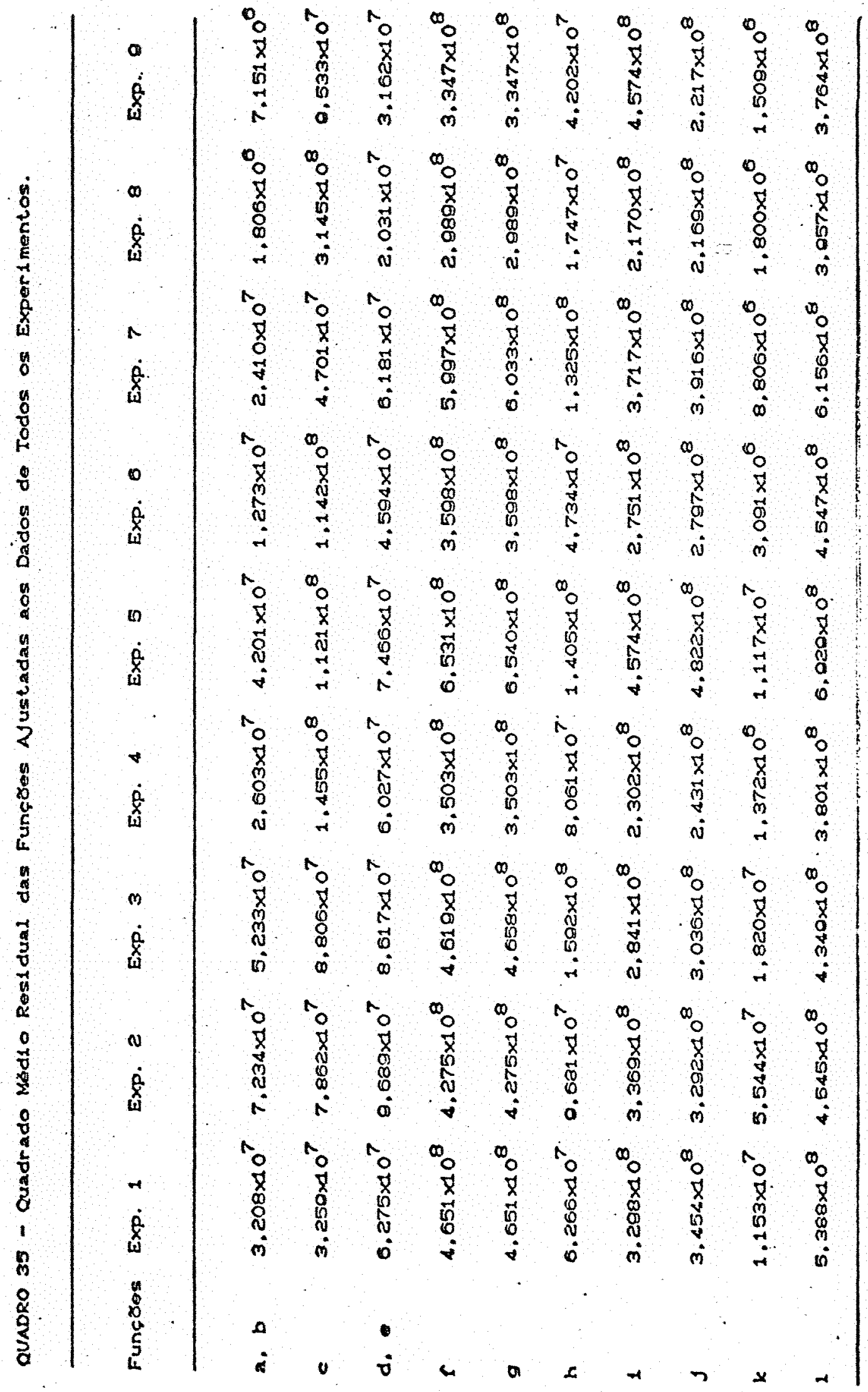




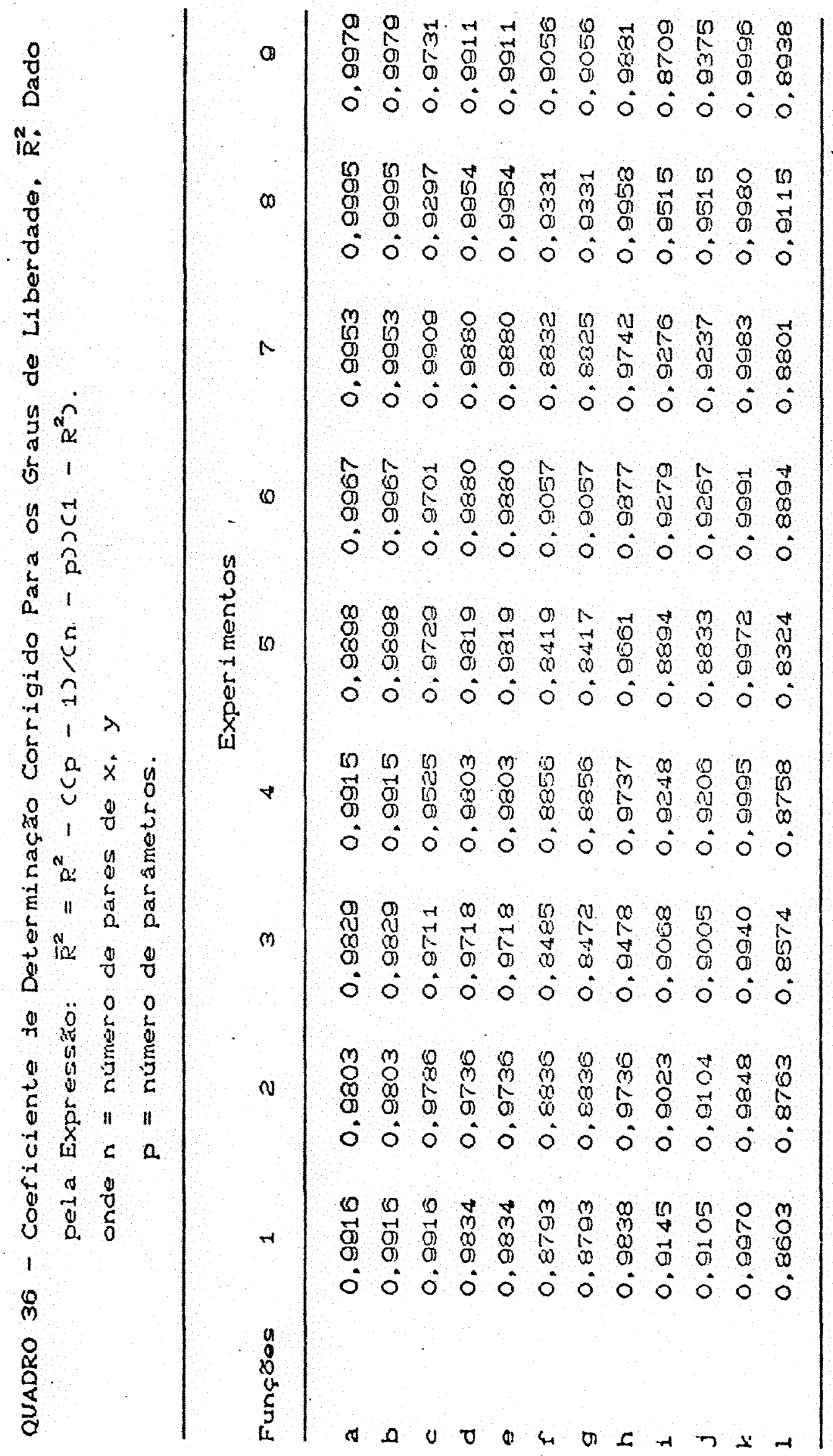


76.

0 Quadro 37 apresenta os valores do Crjterio de Informação de AKAJ KE CAJCD.

QUADRO 37 -. Criterio de Informação de AKATKE Dado Pela Expressáo AIC = ninCSQR + ep

\begin{tabular}{|c|c|c|c|c|c|c|c|c|c|}
\hline \multicolumn{4}{|c|}{ Funçors } & \multicolumn{3}{|c|}{ Fxper i mertos } & \multirow[b]{2}{*}{7} & \multirow[b]{2}{*}{8} & \multirow[b]{2}{*}{9} \\
\hline & 1 & $\approx$ & 3 & 4 & 5 & 6 & & & \\
\hline$a, b$ & 324 & 17770 & 353 & 341 & 349 & 329 & 340 & 296 & 319 \\
\hline$c$ & $3 \pi 4$ & 17830 & $36 \mathrm{a}$ & 370 & 366 & 360 & 351 & 383 & 363 \\
\hline$d, e$ & 334 & 17880 & 861 & 355 & 359 & 351 & 356 & 337 & 344 \\
\hline$f$ & 300 & $190 \mathrm{AS}$ & 380 & 385 & 300 & 386 & 364 & 383 & 385 \\
\hline 9 & 360 & 19049 & 390 & 385 & 380 & 386 & 395 & 380 & 389 \\
\hline$h$ & 334 & 17980 & $37 \%$ & 360 & 370 & 351 & 360 & 354 & 340 \\
\hline$i$ & 560 & 18878 & 381 & 377 & 388 & 380 & 386 & 370 & 380 \\
\hline$j$ & $36 \%$ & 18861 & 383 & 370 & 381 & 381 & 387 & 377 & 378 \\
\hline$k$ & sos & 17579 & 336 & 290 & 328 & 300 & 323 & 296 & 293 \\
\hline$i$ & 368 & 19094 & 390 & 387 & 398 & 391 & 390 & 388 & 387 \\
\hline
\end{tabular}

Os. vajores de AIC para o Experimento e são matores que os demais fxperimentos. Isto porque neste Experimento o valor de $r$ major fara o mesmo Experimento podemos comparar os ajustes das funçoes. 0 menor valor de AIC representa o mel hor ajuste. 
Com base nos quadractos medjos residuais, COMR foi atribuido, a cada uma das doze funçóes estudadas, seu posto em rejaçáo as demais. Atribuiu posto 1 a furcia que apresento meror $C O M k$ os resultados aparecem no Quadro 38 .

QUAlbko 38 - Frequémela dos Fostos de cada Funçäo, em Relaço às Demais, de Acordo com o OMF

Funçes

Postos Total E fipi 13345070101112

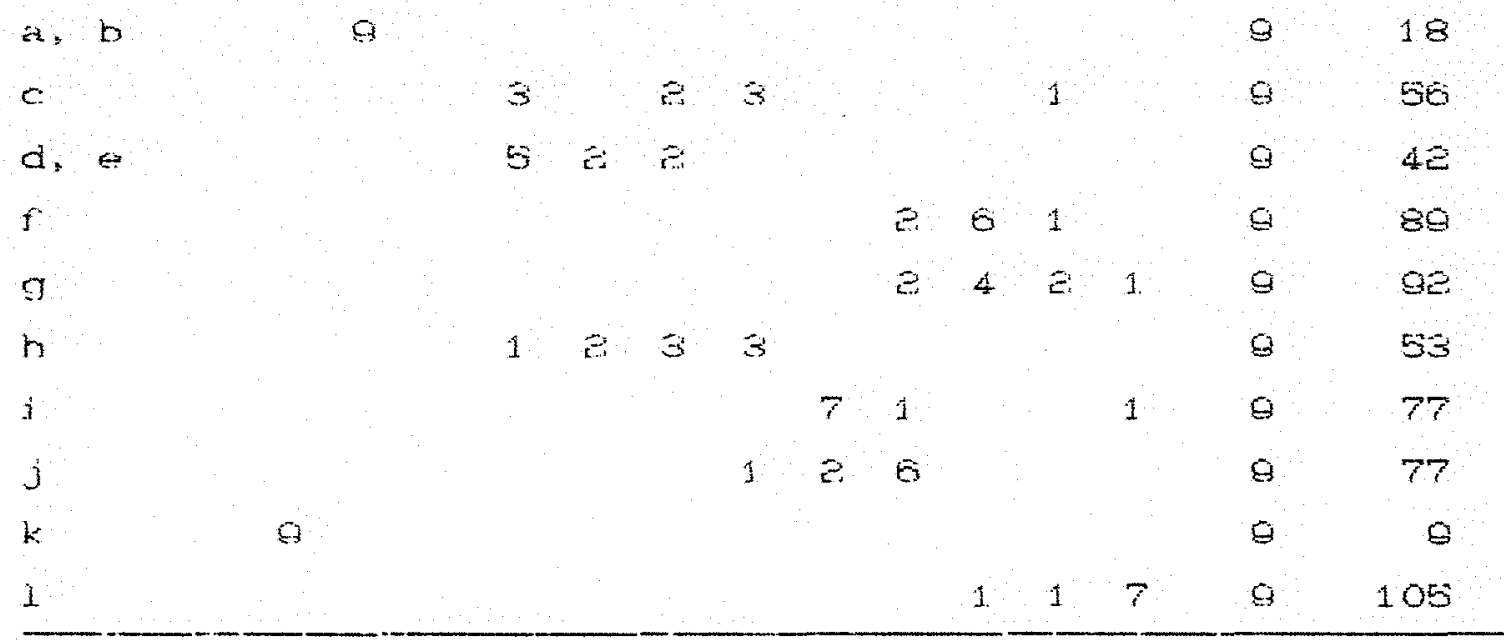

Observa-se que a funçăo kjchards cks apresentiou o menor COMR em todos os Experimentos,

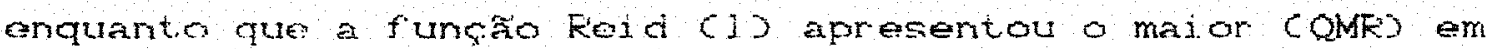
sete Experi mentos. 
Foram atribul dos postos as funços em cada experimentio, em relacico ac coeficiente de determinagão corrigido $\left(R^{2}\right)$, posto 1 para a funça que apresentou maior val or de $\bar{k}^{2}$ os resul tados estao no quadro 39 .

QUAHKO 39 - Frequencia dos postos de cada funça em Relactio dis Deines, de Acordo com o Coef jciente de Determi nação Corrigido $\bar{R}^{2}$

\begin{tabular}{|c|c|c|c|c|c|c|c|c|c|c|c|c|c|c|}
\hline \multirow[t]{2}{*}{ Funçöes } & \multicolumn{12}{|c|}{ Postos } & \multirow[t]{2}{*}{ Total } & \multirow[t]{2}{*}{$\Sigma$ fipi } \\
\hline & 1 & $\vec{r}$ & 3 & 4 & 5 & 6 & 7 & 8 & 9 & 10 & 11 & 12 & & \\
\hline$a, b$ & & 9 & & & & & & & & & & & 9 & 18 \\
\hline$c$ & & 1 & & 2 & & 2 & 3 & & & & 1 & & 9 & 54 \\
\hline$d, e$ & & & & 6 & 4 & & & & & & & & 9 & 40 \\
\hline$f$ & & & & & & & & & 3 & 6 & 1 & & 9 & 89 \\
\hline 9 & & & & & & & & & $\therefore$ & 4 & 2 & 1 & $\theta$ & $\theta e$ \\
\hline n & & & & 1 & 1 & 3 & 4 & & & & & & 9 & 55 \\
\hline$j$ & & & & & & & 1. & 6 & 1 & & & 1 & 9 & 76 \\
\hline$j$ & & & & & & & 1 & 2 & 6 & & & & 9 & 77 \\
\hline$k$ & $\theta$ & & & & & & & & & & & & 9 & 9 \\
\hline 3 & & & & & & & & & & 1 & 1 & 7 & 9 & 105 \\
\hline
\end{tabular}

0 quadro 39 mostra, que tambem com relaça ao $\overline{\mathrm{F}}^{2}$, a funcao de Eichards $(\mathrm{k})$ fol a que mel hor se ajustou aos dados de todos os Experimentos a o pior ajuste fol o da funça keld CI3. 
78.

No Quadro 40 estrio os resultados da atributgáo de postos as funcóes, com relaça ao oriterio de informaçăo de Akajke (AICS, posto 1 para a função que aprentou menor valor de AIC em cada experimento.

QUADRO 10 - Frequencia dos postos de cada Funçăo em Relaçáo as Demais, de Acorcio com o Criterio de Informas $\mathrm{xo}$ de AKATKE CAICO

Funçies

Postos

Total $\sum$ fip

12845678 \& 8101112

\begin{tabular}{|c|c|c|c|c|c|c|c|c|c|c|c|c|c|}
\hline$a, b$ & 1 & 8 & & & & & & & & & & 9 & 17 \\
\hline$c$ & & 1 & 2 & & $\approx$ & 3 & & 1 & & & & 9 & 58 \\
\hline$d_{1} \theta$ & & & $\theta$ & 3 & & & & & & & & $\Theta$ & 39 \\
\hline$\hat{r}$ & & & & & & & & $\hat{c}$ & 7 & & & 9 & 88 \\
\hline 9 & & & & & & & & $E$ & $\theta$ & 1 & & 9 & 89 \\
\hline $\mathrm{r}$ & & & 1 & 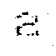 & 3 & 3 & & & & & & 9 & 53 \\
\hline$i$ & & & & & & 1 & $\theta$ & 1 & & & 1 & $\theta$ & $7 \theta$ \\
\hline $\mathrm{J}$ & & & & & & & 3 & 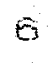 & & & & 0 & 78 \\
\hline$k$ & $\theta$ & & & & & & & & & & & 0 & 9 \\
\hline 8 & & & & & & & & & 1 & 1 & 7 & 9 & 105 \\
\hline
\end{tabular}

Com base na estatjstica $I$ fipi dos Quadros 38, 39 \& 40 , pode-se observar que os resultados são muto parecidos. For exemplo, a funça de Eichards $C k J$ apresenta, nos tres quadros, o menor valor de $\Sigma f i p j$, enguanto que a de Feid CJ apresenta o maior valor. Isto mostra uma concordancia de resultados dos eriterios adotados. 
80.

5. CONCLUSEES

Em situaçós como a cleste trabajno em que se estudam varias funsoes ajustadas a diversas formas de crescimento, cspera-se que uma boa fungäo terha as seguintes caracteristicas:

- 12 Que apreserte um bom ajuste a cada conjunto des dados:

- Do Que tenna a flexibilidade de se ajustar bem na majorla dos experimentos;

- 3) Que consiga atu rigir a convergencia a partir de valores iniciajs năo muito proximos das estimativas dos par ametros:

- 4) Que encontre o ponto de convergencia em poucas i ter acóses.

Tendo em vistas estas caracteristicas podem-se tirar a) gumas conclusóes a respeito das funços estudadas neste trabalno.

1) A runçáo de Richards, dada pela expressáo $(k)$ y $=\beta_{0}\left(1 \pm \beta_{1} e^{-\beta} x_{2} \gamma_{3}\right.$, ajustou-se mel hor que todas as demals em todos os experimentos. Tal functo năc exige muto da precisfio dos valores injojals dos parametros; converge sempre para o mesmo ponto de mínimo 
81.

de funçio $\xi$; flexivel ao ajustar-se bem as diferentes formas de crescimento. Fsta funcáo apenas necessi ta mui tas iteraçoes ate atjugir a convergencia 0 que nád $e$ ná verdade, um arande problema dada a facilidade de utilizará a a erictencia da jnformatica atual.

P) A funçio logistica (nas formas $a$, b) apresentou o segundo melhor ajuste em todos us exper imentos. A funciao na forma $\mathrm{CO}$ tambem mostrou ajuste como na $(a) e$ na $(b)$, desde que os valores inicials ti versem sido conveni entemente escolhidos, isto o, o valor inicial para $\beta_{1}$ fosse iqual a $-1 n C \beta_{1}$ o festa funça $c c$ mostrou ser muto exi gonte quanto a precisäo dos vajores inicuats, o que andesejavol. 0 tato de alcancar a convergencia em pontos diferentes conforme sejam diferentes os valores injlias dos parametros, del xa o pescyusadm sem saber se o ponto alcangado $e$ o de minmo absolutio ou tocal, fortanto, deve-se ter cautel a ao usar esta fumbio mar representar curvas de cresejmento.

3) A functo de Gompertz Cformas d, es tambem aprosentou hom ajuste ficarido logo atras da funfäo 1 ogistica Fste fato confirma o que ja t bastante combeldo com relarko a eficiencia dessag turródes para descrever curves de erescimento.

4) A funcfor logistica Modificada Ch ajustou-se razoavel mente bem na maiorla dos experimentos. Fsta funçio deve constar dentre aquelas usadas para ajustar a dados de crescimento, pols, alem de ná exigir mut ta precisfo dos valores injejals, ajusta-se muito bem a diversas formas de crescimento. 
5 As funços (i) Exponencial e (j) Iog-inversa-generalizada náo se ajustaram bem em quase todos os experimentos, apesar de serem funços assintitions sigmoides, caracteristicas de curvas de cressci mento.

6) As funçes (f) monomolecular e Coj Brody nấo se ajustaram bem ra maloria dos experimentos. Isto contraria a afirmafio de RTCHARDS (1959), que cita a monomolecular como uma das tres funçes que melhor descrevem curvas de croscimento, juntamente con a logistice e a de compertz.

7) Finalmente a funça de keld clo fol a que apresentou $\mathrm{a}$ plor ajuste em quase todos os experimentos. Fista funça possuj quatro parametros, o que diflouta a escolha dos valores indejals. Fara valores inidals diferentes, quase sempre a convergencia e alcancada om pontos diferentes. Isto obriga o pesqui sador a testar varios valores injejais, ate atingir o minimo absoluto. Das caracteristicas desejavejs, a funçáo de keid apresentou apenas uma, ou seja, rapidez na convergeneia. Isto, na verdade, nac significa muto, pois nem sempre o ponto atinglo $\theta d$ de minimo abroluto porque depende basicamente da escolha dos valores injeiais. 
REFEREINCTAS BTBI YOGNAFYCAS

ANUARTO ESTATISTTCO DO BRASTH, KIO dE JAREIRO, V. 49 , $19839.716 \mathrm{p}$.

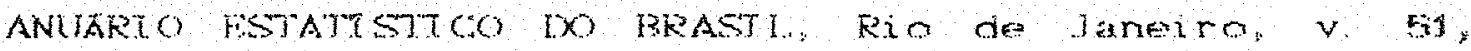
1.991. $1004 \mathrm{p}$.

AKAlKE, H. A new look at the statistical modei i centifietion. YFEF Trarsactions of Automatic Control.

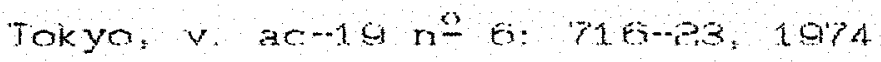

ARKIDA, H. V.; VIELRA, S.; HOWFMANN, R. AnAJ.jse de uma funçie sigmoldes Arquive do Tnstituto Biologico, sao pauto 4O: 14-R, 1978.

GALSTON, D. R. A computer program for rjttjng the kishards

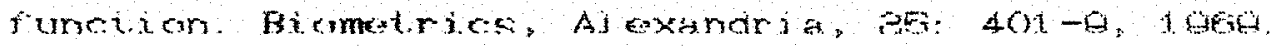

CORTAREIII, A. Estudo da curva de crescimento de machos de raba Nelese, atraves de quatro modeles estacasticos. Jaboticerbl, 1973,179 p Conutorado-faculdade de Medi cina Veterinaria 6 Agricul tura de Jaboti cabaj LNFSP

TRAPER, N, \& SMTTH, H. APpl ied regression anal ysis. $2 \underline{\text { ed }}$ New York, Jom Wi loy, test $40 \% \mathrm{p}$

GALLANT, R A Nonlinemer statistical morels. New lork. Ionn witey, $198 \%$. $610 \mathrm{r}$. 
84.

HOFFMANN, R, \& THAME, A C. M Doterminargo da idade dima para primeiro desbaste em povoamento de pinus

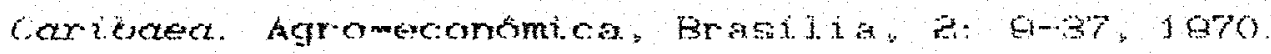

MAONANT, R. Alguns aspectos da apj i carä́ da segunda les de Mitsenertich $\theta$ da equats de regression quadrated a

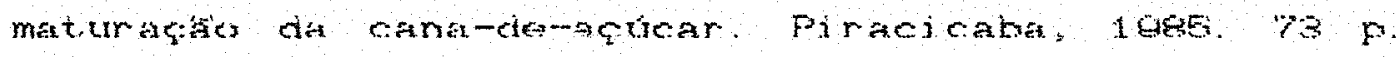
CMestrado - Fscola Sugerior de Agricultura "lubz de Queirom" Nisps

MARQUARIJT, D. An algorithm for least-squares estimation of neml tnear parameters. Jotrnal society for Industrial and Applled Mathematics, Philadel phis, 2, 431-41, 1963 .

MSCHAN, M. M. Anelise ecencmetrica do erescimento de gado bovino. Botucatu, 1 Gre CIoutorado - Faculdade do

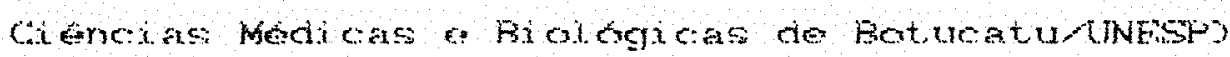

NEIJFR, J. A. The fitting of a gereralization of the logistics corve Hometrios, Alenxandis, 17 : $89-110$. 1001 .

OLIVER, F. R. Asperts of maximum itiketinnod estimation or the lesichtic growt function Jourral of the American Statatistical Association, Boston 61 : $6 \mathrm{Q} 7-705,1966$.

OIVETRA, $M$. $A$ de Comparagio de modelos matematicos aphicados on enalos de ojagem com a

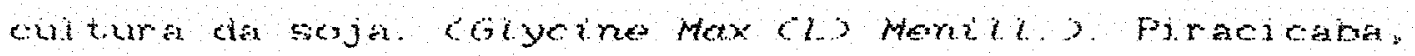
1986, teg p. CMestrado - Escola Superjor do Agricultura "Luxp de Ous row" wesp 
85 .

RTCHARDS F. I. A fiexible growtin runction for empirical. use?. Joumnal of Experimental Botany, Cxan, 10 : $200-300,10503$.

RTCHARDS, F. $S$ G. A method of maximum-likejinood estimation. Joumal Royal of Statistical Socinty $\mathrm{f}$, $1.0 \mathrm{xam}, \mathrm{a}, 469-75,196 \mathrm{~d}$.

STLVA, M. A. P. Metorlos de estimagĩo de parametros do modelo $y=a\left[1-10^{-\mathrm{cm}_{1}} \mathrm{O}^{-\mathrm{k}^{2} \mathrm{~m}}\right.$ aplicados a dados de

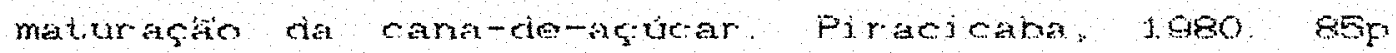

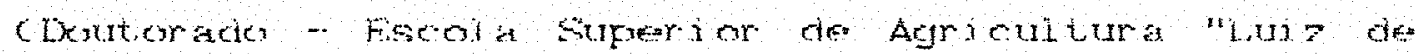
Quenroz" ASP?

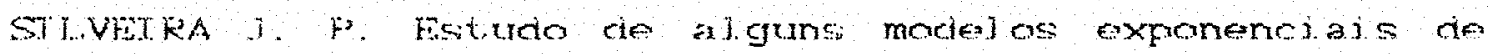
cresel mento de bovinos de ract thage. Piracieate 1070.

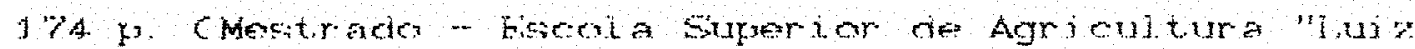
de Geiroz"nisp

SPARROW, P. E. The comparison af it ve response curves ior representing the retertionship betweer the arrual. dry-matter yield of grass herbage and fertulizer nitrogen Journal of Agricultural solence Cambridge. 93. $513-30,1979$

STHVENS, W. L. Asymptot, Le regression Biometrics: Alexendrata, $747-67,1051$.

THOKNIFY, 3. H. M. Mathematical models in plant

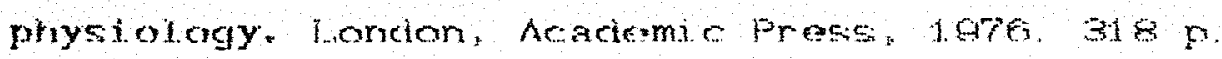

WT LSON, A. I. \& DOUKLAS, A. W. A note on nonlinear curve istung. Amoricar statisticiar, washington, 33 : $37-8$, 1063 . 
86.

VERHULST, P. F. Recherches mathematjquen sur joj

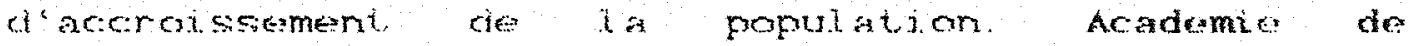
Frorselles, $14,1845$.

VIFIRA, S. Fistudo das funches assintotico-sigmoides.

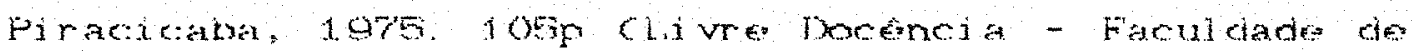

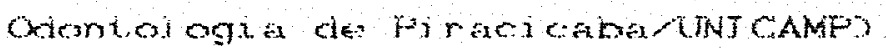


87.

APFINDTCE 
88.

TABEl 3. Fxperimento 3 - Peso em kgha da massa verde da cana-de-actuar coana-soca). reali rado em Firacicaba em $198 \mathrm{r}$,

Experimento

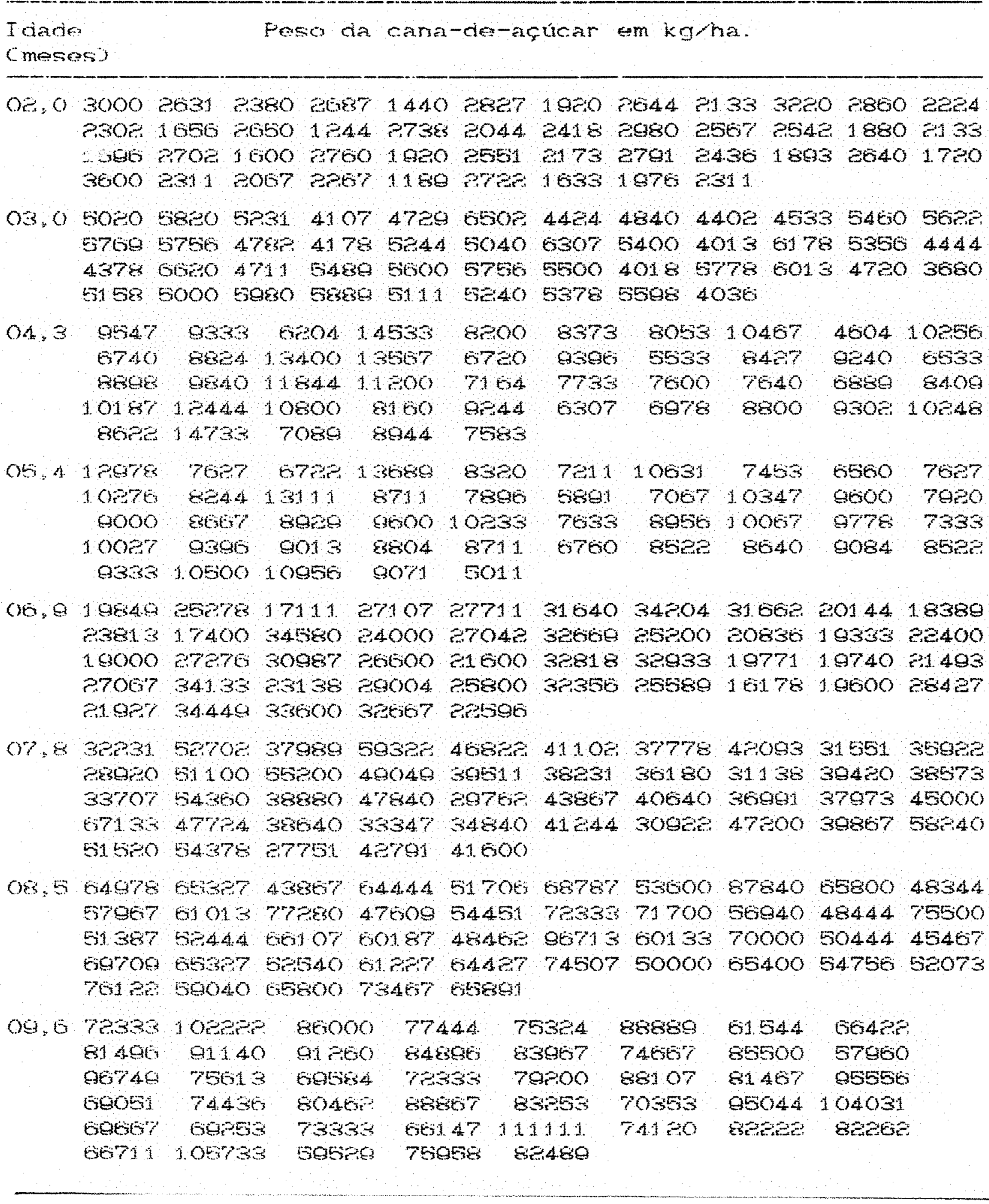


TABRLA 3. Experi mento 2 - Feso em $\mathrm{kg}$ ha da massa verde da cana-de-acucar (cana-soca). Experimento real izado em Firacicaba em 1681,8 .

\author{
Tolade \\ Feso da cana-de-acúar em koha \\ c meses
}
10.0127344 126827 138367 128778 86133 j37244 103333137318 100667103756137078 158278 120339 123067 91733143860 $105667 \quad 0333313940$ 106027 155800117111124667114333 128000107120103300138700 1.76187 les578 121000131556 $101333 \quad 148000$ 106333 85344 136807 $131413 \quad 143078$ 127556 $13170413,53 \quad 104560100080112667$

$11,6143000 \quad 139780 \quad 144786 \quad 145387$ 144987 142078 139760145987

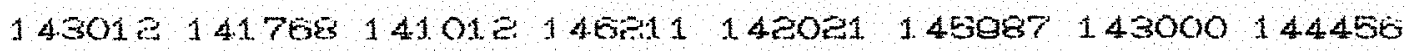
139876 1 48345 146789 139876 $147912143356 \quad 144234 \quad 143334$ $142100143012 \quad 142987 \quad 146876 \quad 145567 \quad 141980 \quad 142334 \quad 144000$ 141987 142987 $145 \% 43 \quad 143524 \quad 147123$ 1 $41846 \quad 145608$ 142897 $143615143080141987 \quad 143718142560$

$12,6146000144956 \quad 446243 \quad 143756 \quad 144867 \quad 145837 \quad 144921 \quad 143958$

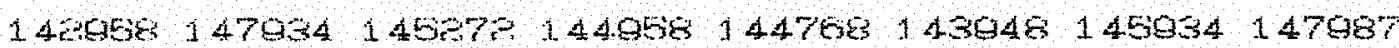
140768 148789 143989 147987 $146123 \quad 145739148789146453$ 146458 147133 144876 149879145438 146879 144876143987

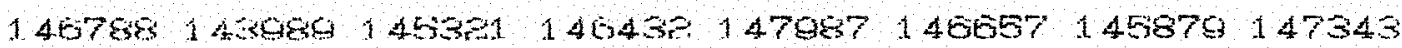
$146897 / 45087 \quad 147381 \quad 144987 \quad 145500$

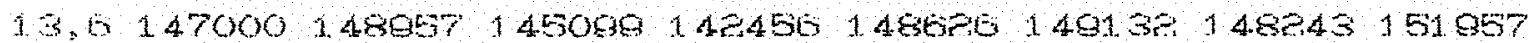

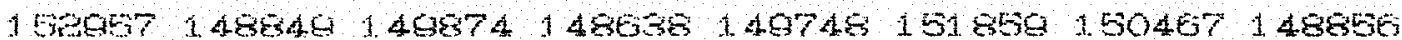

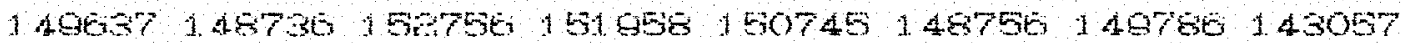
$147658 \quad 160987$ 149876 148764 a 46345 153758 $148356 \quad 149674$

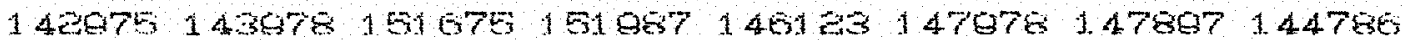
140870,143133150343151679149780

1. 4.6146000148984 144407 144897 149895 148984 15F859 145908

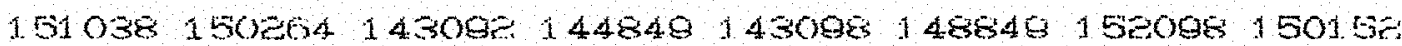

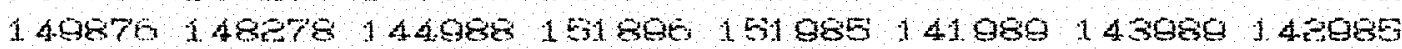

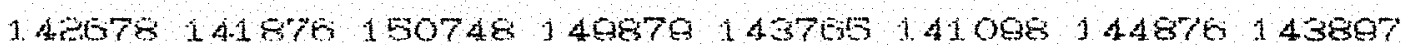
150499 150098 146869 147875 143859 147896 $158957 \quad 144819$ 151830 1.88\% 143930 1474.86 150617

$15,6140000144867 \quad 147034 \quad 146786,145987 \quad 147133 \quad 147067 \quad 145786$

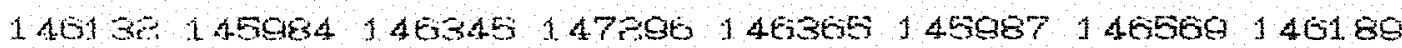
$145879140784 \quad 145897 \quad 147834 \quad 146892147198146056145871$ 145081 146789 147433 146689 145897 146E96 146780147098 147178147868 i 45867 14587\% 146893147840 1455EQ 147349

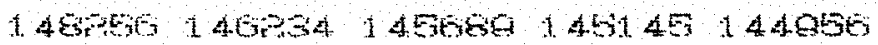

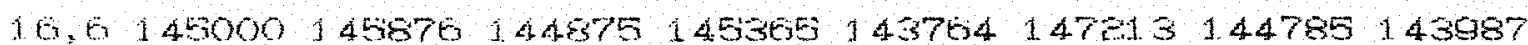
147301 146489 145457 145897 143896 144786 146350147320

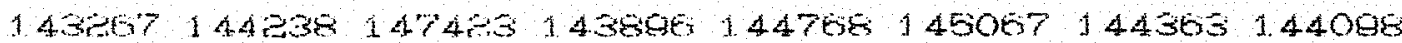

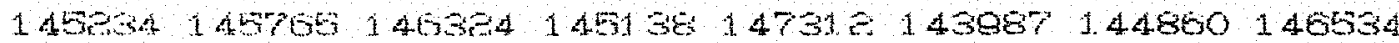

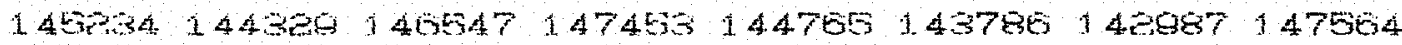
143456145780 144673 147174144678

$17,6148000143450142567 \quad 144698141986$ 144658 142689143345 144560 i 41738 145724 143078 1 44237 1 42278 1 46583144087 142678 141789 145780 143456 142957 1 48350 141456 143678 $143567 \quad 143894$ 144704 142560 141456 145660 143457 143786

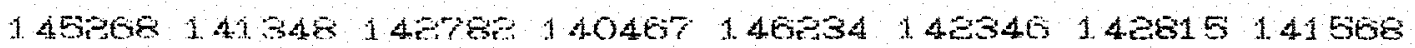
$146678144023143089+44131+41534$ 